\title{
Astronomical Distance Determination in the Space Age Secondary Distance Indicators
}

\author{
Bożena Czerny ${ }^{1} \cdot$ Rachael Beaton $^{2} \cdot$ Michał Bejger $^{3} \cdot$ Edward Cackett $^{4}$. \\ Massimo Dall'Ora ${ }^{5}$ R.F.L. Holanda ${ }^{6} \cdot$ Joseph B. Jensen $^{7} \cdot$ Saurabh W. Jha ${ }^{8}$. \\ Elisabeta Lusso, $^{9,10}$ • Takeo Minezaki ${ }^{11}$ - Guido Risaliti ${ }^{9}$ Maurizio Salaris ${ }^{12}$. \\ Silvia Toonen ${ }^{13}$. Yuzuru Yoshii ${ }^{11,14,15}$
}

Received: 3 July 2017 / Accepted: 3 January 2018 / Published online: 23 January 2018

(C) The Author(s) 2018. This article is published with open access at Springerlink.com

\begin{abstract}
The formal division of the distance indicators into primary and secondary leads to difficulties in description of methods which can actually be used in two ways: with, and without the support of the other methods for scaling. Thus instead of concentrating on the scaling requirement we concentrate on all methods of distance determination to extragalactic sources which are designated, at least formally, to use for individual sources. Among those, the Supernovae Ia is clearly the leader due to its enormous success in determina-
\end{abstract}

Astronomical Distance Determination in the Space Age

Edited by Richard de Grijs and Maurizio Falanga

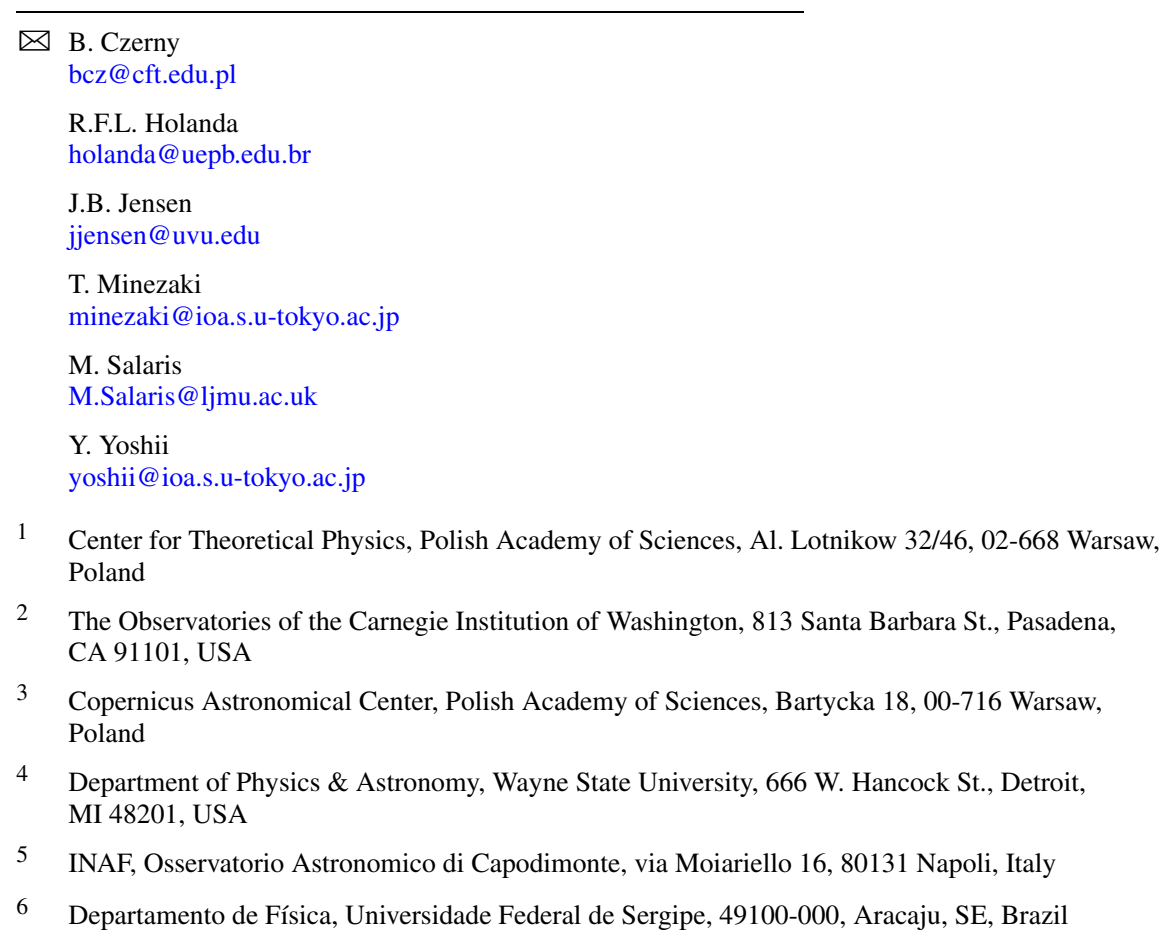


tion of the expansion rate of the Universe. However, new methods are rapidly developing, and there is also a progress in more traditional methods. We give a general overview of the methods but we mostly concentrate on the most recent developments in each field, and future expectations.

Keywords Distance scale - Galaxies: distances and redshifts - Stars: supernovae

\section{Introduction}

Measurement of the distance to extragalactic objects is an important task. For objects at large distances the measurement of the redshift was frequently considered as the measurement of the distance but precision cosmology requires the knowledge of both the distance and the redshift.

The measurement of the distances to extragalactic objects was for years based on a carefully set subsequent steps of the cosmic ladder: direct distance measurement methods allowed to formulate scaling relations which then could be applied to more distant objects, and with a few such steps cosmological distances were achieved. The fundamental direct method of measuring the distance in astronomy is to measure the trigonometric parallax of a given source, reflecting the motion of the Earth around the Sun. Hipparcos mission measured parallaxes up to a few hundred parsecs, Hubble Space Telescope reached up to $5 \mathrm{kpc}$ in dedicated observations. Now Gaia mission is collecting the data. It will cover with precisions of $10 \%$ a volume of radius $10 \mathrm{kpc}$, which encompasses $<1 \%$ of the stars in our Galaxy by number, but by volume is very large and will cover a large fraction of the Milky Way with reasonable accuracy. Parallaxes of the Cepheid stars, combined with measurements in nearby galaxies, calibrate the Leavitt Law for Cepheids, and Cepheids are accessible to $30 \mathrm{Mpc}$ with the Hubble Space Telescope. Further out, SNe Ia calibrated with the help of the Cepheids take over, covering cosmological distances up to the redshift $\sim 2$. This method replaced older methods of covering most distant part of the ladder, like Tully-Fisher relation.

Modern astronomy still strongly relies on indirect measurements which in turn rely in some intermediate steps but in the aim to meet expectations of the precision cosmology the

7 Utah Valley University, Orem, UT 84058, USA

8 Department of Physics and Astronomy, The State University of New Jersey, Piscataway, NJ 08854, USA

9 INAF, Arcetri Astrophysical Observatory, Largo E. Fermi 5, 50125 Firenze, Italy

10 Centre for Extragalactic Astronomy, Department of Physics, Durham University, South Road, Durham, DH1 3LE, UK

11 Institute of Astronomy, Graduate School of Science, The University of Tokyo, 2-21-1 Osawa, Mitaka, Tokyo 181-0015, Japan

12 Astrophysics Research Institute, Liverpool John Moores University, IC2, Liverpool Science Park, 146 Brownlow Hill, Liverpool L3 5RF, UK

13 Anton Pannekoek Institute for Astronomy, University of Amsterdam, 1090 GE Amsterdam, The Netherlands

14 Steward Observatory, University of Arizona, 933 North Cherry Avenue, Room N204, Tucson, AZ 85721-0065, USA

15 Institute for Cosmic Ray Research, The University of Tokyo, 5-1-5 Kashiwa-no-Ha, Kashiwa City, Chiba 277-8582, Japan 
intermediate steps are refined, new shortcut developed and some of the basically indirect methods might be potentially used as direct methods under some physically well motivated assumptions. In the Local Group the use of the eclipsing binary stars allows to skip the step of the parallax measurement. A very important direct distance measurement is possible for the spatially resolved water maser in the galaxy NGC 4258. The impressive jump directly to very large distances opened with observations of gravitational lensing of quasars and the measurement of the time delays between the quasar images which allows their use as a ruler. Another direct method can be applied to galaxy clusters. This method uses the SunyeavZeldovich effect. Extremely important direct methods are the Barion Acoustic Oscillation method and the observations of the fluctuations of the Cosmic Microwave Background. In both cases the (statistical) analysis of the sizes of the fluctuations allows to get directly the cosmological constraints. Another statistical method to obtain the cosmological constraints is gravitational lensing. Now, with firm detection of gravitational waves, the possibility of the direct determination of the distance to the gravitational wave source from the shape of its signal also gains importance. All these methods are described below.

We do not follow the strict definition of indirect method but in this section we concentrate on predominantly indirect methods which can be applied to single specific extragalactic $o b$ jects like supernovae, galaxies, galaxy clusters, active galaxies or gamma-ray bursts. Global statistical methods, including the results based on the Cosmic Microwave Background, will be discussed in a separate Chapter. We also do not include in this Chapter strong gravitational lensing since it will be more natural to address this issue in the Chapter where weak lensing is discussed.

We outline the methods shortly, with references to the basic books or reviews, and we concentrate on most recent developments in the field. Whenever possible, we discuss the issue of the method cross-calibrations.

The order of the methods' presentation is roughly adjusted to their past and current popularity and role in astronomy, but not necessarily reflects their potential for development in the near future.

\section{Supernovae Ia}

Type Ia supernovae (SNe Ia) are defined by the lack of hydrogen and helium lines around the maximum light and the presence of strong SiII absorption features. Their peak absolute magnitudes are bright $\left(M_{B}\right.$ typically between -18.5 and $\left.-19.5 \mathrm{mag}\right)$ and moreover for the majority of them (about $64 \%$ of all SN Ia events, denoted as 'normal', see Li et al. 2001) the light curves are remarkably similar, in the sense that they display a well defined relation between the peak absolute brightness and the width of their lightcurve (Phillips 1993). This characteristic makes SN Ia events very good cosmological distance indicators, and led to the discovery of the accelerated expansion of the universe (Riess et al. 1998a; Perlmutter et al. 1999a).

SNe Ia are intrinsically luminous (Riess et al. 2016), thus, even with modest aperture telescopes SNe Ia can be discovered over large volumes; indeed, the All Sky Survey for Supernova (ASAS-SN; Shappee et al. 2014a) uses telescopes with only a $14 \mathrm{~cm}$ aperture to discover a total of 242 confirmed SNe, of which 183 were SN Ia (76\%) over its first 18 months of dual-hemisphere operations (01 May 2014 to 31 December 2015) alone within $m_{V}<17$ (Holoien et al. 2017a). ${ }^{1}$ Their intrinsic luminosity also permits their discovery

\footnotetext{
${ }^{1}$ See also Holoien et al. (2017d) and Holoien et al. (2017b) for the 2013-2014 and 2016 statistics, respectively.
} 


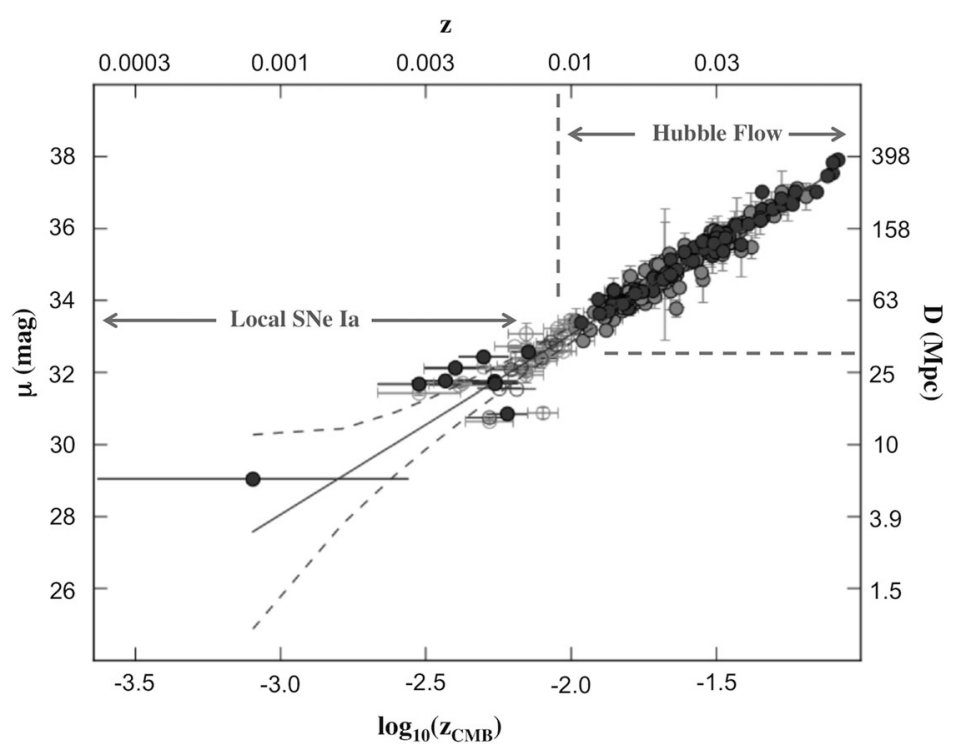

Fig. 1 Hubble diagram for SN Ia in the Carnegie Supernova Project (dark grey) and the CfA4 survey (grey). The scatter for the SN Ia in the 'Hubble Flow' sample $(z>0.01)$ is 0.12 mag or $6 \%$ in distance, which when averaged over the $200 \mathrm{SN}$ Ia in the sample, results in a total uncertainty of $0.7 \%$ (Beaton et al. 2016). The effect of peculiar motions on the 'local' sample are evident by their large scatter. The 'local' sample is far less complete, with only eight galaxies in this visualization; Riess et al. (2016) brought another 11 SN Ia into the 'local' sample. (Adapted from Beaton et al. 2016)

at significant redshift; the previous most distant SN Ia SN UDS10Wil at $z=1.914$, which was discovered in the Cosmic Assembly Near-infrared Deep Extragalactic Legacy Survey (CANDELS, PI: Faber \& Ferguson; see Jones et al. 2013, for details), was recently replaced with another 15 in the range from $1.9<z<2.3$ presented in Riess et al. (2017). SNe Ia in the redshift range from $0.2<z<0.8$ are now routinely discovered and used for cosmological purposes; in the first two years of the ESSENCE project, 52 SNe Ia were discovered in this redshift range (Matheson et al. 2005).

SNe Ia, however, are not trivial objects to study and understand. Paramount among the difficulties of SNe Ia is that their progenitors and the exact physics of their ignition mechanisms are actively debated (see Sect. 2.7). Despite this, empirical measurements of the SNe Ia, as a class, show remarkable homogeneity and, due to their frequency and ubiquity, $\mathrm{SNe}$ Ia are likely to remain our tools for an en masse probe of the distant Universe. Figure 1 is a Hubble Diagram, a plot of distance versus redshift, for SNe Ia within $z<0.1$ that demonstrates the remarkable precision $\left(\sigma_{\mathrm{SNeIa}} \sim 0.12 \mathrm{mag}\right.$ or $6 \%$ in distance $)$ of SNe Ia as a class. With a sample size of $>200$ (and growing), this stage of the extra-galactic distance scale is, by far, the most well constrained and provides a random uncertainty of $\sim 0.7 \%$ to the determination of the local expansion rate of the Universe (the Hubble constant or $H_{0}$ ) (for details see discussion in Beaton et al. 2016). In this Section, the key observational components and considerations required to construct the dataset in Fig. 1 are described.

\subsection{Early History of SNe}

Wood-Vasey et al. (2007) present a short contextual history of the role of SNe for cosmology that we will expand upon herein. Within the context of the 'Great Debate', Shapley (1919) 
argued that intrinsic brightness of SN 1885A found within the Andromeda galaxy of $M=$ -16 mag was "out of the question" if Andromeda was, indeed, an 'extragalactic nebulae.' Thus, Shapley argued, the island universe hypothesis for the nature of the nebulae was likely incorrect owing to this extreme luminosity. In his landmark paper describing the Cepheid variables discovered in Andromeda between 1923-1928, (Hubble 1929) commented on the existence of "a mysterious class of exceptional novae which attain luminosities that are respectable fractions of the total luminosities of the systems in which they appear." Since Hubble's work provided strong evidence that the nebulae were, indeed, extra-galactic in nature (Code 1999), this new class of variables was later classified as 'super-novae' by Baade and Zwicky (1934) because the approximate amount of energy released by these objects over 25 days "is equivalent to $10^{7}$ years of solar radiation of the present strength."

Minkowski (1941) was the first to apply a classification scheme to the supernovae, which was based on the presence or absence of hydrogen emission lines in the spectra (the type II and type I, designations respectively). As Minkowski notes, the spectra for SNe of type I seemed remarkably homogeneous in support of the postulation by Wilson (1939) that such objects could be employed for cosmological exploration. Indeed, the discovery and utilization of SNe for distances relies on their photometric properties, but the classification of $\mathrm{SNe}$ into phenomenological subtypes is largely reliant on their spectroscopy. The dual-need of spectroscopic and photometric classification is a challenge for the accumulations of the anticipated large samples to be discovered in the 2020's by large scale transient monitoring programs like the Large Synoptic Survey Telescope (LSST; see discussions in Matheson et al. 2013; LSST Science Collaboration 2009).

Moreover, with the advent of long-term spectroscopic and photometric monitoring of a large number of SNe Ia, there are now additional sub-classifications based on both spectroscopic and photometric diagnostics (see discussion in Parrent et al. 2014).

While both our theoretical understanding and experimental census of SNe Ia has increased dramatically, many of the original mysteries regarding the SNe Ia posed by Baade and Zwicky (1934), when their class was initially defined remain, as vibrant areas of research nearly a century later.

\subsection{SN Ia as Standardizeable Candles}

While their early homogeneity was suggestive of their utility as standard candles as early as Wilson (1939), the ability to be used as such occurred only in the last 30 years. Phillips (1993), expanding on earlier work by Pskovskii (1977), reported on an apparent empirical correlation between the absolute luminosity of a SN Ia and its the rate-of-decline in optical lightcurves $(B V I)$. The decline rate is the change in brightness of a given band over the 15 days post maximum and is designated $\Delta m_{15}(\lambda)$; a typical decline rate is $\Delta m_{15}(\lambda)=1$, but rates can vary between $0.9<\Delta m_{15}(\lambda)<1.8$ (Phillips 2012). The $\Delta m_{15}$ parameter is relatively straight forward to measure with appropriate light curves and acts as a scale factor through which light curves of SN Ia are standardized and used as distance indicators. In works by Hamuy et al. (1995) and Riess et al. (1996), it was clear that SNe Ia corrected for their decline rate provided precise distances.

The physics underlying this relationship is that intrinsically brighter SNe Ia take longer to fade than intrinsically fainter SNe Ia and this is largely thought to be due to different amounts of ${ }^{56} \mathrm{Ni}$ contributing to the early time light curve, though the reason for this could be due to differences in either the total mass or its distribution in the explosion (see Kasen and Woosley 2007; Parrent et al. 2014, for detailed discussions). Recent energy has been focused on the near-infrared properties of SNe Ia, which show far more homogeneity than 
the optical and are less sensitive to dust (a detailed review is given by Phillips 2012). Indeed, in Fig. 1, the dark grey points from the Carnegie Supernova Project (CSP) show a tighter dispersion than those in grey from Harvard-Smithsonian Center for Astrophysics (CfA4), precisely because of the CSP use of near-infrared photometry.

Since the large scale demonstration of SNe Ia as standardizable candles, they have been studied at a number of regimes; these are: (i) local $(z<0.03)$, (ii) intermediate $(0.03<z<$ $0.3)$, (iii) distant $(z>0.3)$, and (iv) high- $z(z>1.2)$. The SN Ia discovered within each regime are used for different goals. Local SNe Ia can simultaneously constrain progenitor properties and serve as calibration sources. Intermediate $\mathrm{SNe}$ Ia are safely in the Hubble Flow and used to measure $H_{0}$. Distant and high- $z$ samples are used to trace the acceleration of the Universe $(w)$. Thus, large collaborations have been formed to find, study, and use SNe Ia and these generally separate into redshift (and brightness) regimes.

Amongst numerous efforts in the community, several of note are as follows: the CfA survey (Riess et al. 1999; Jha et al. 2006; Hicken et al. 2009), the Carnegie Supernova Project (CSP Hamuy et al. 2006; Freedman et al. 2009; Folatelli et al. 2010), ESSENCE (Miknaitis et al. 2007; Wood-Vasey et al. 2007; Foley et al. 2008, 2009), Higher-z Team and SHOES (Riess et al. 2004b,a, 2007, 2009), the Supernova Legacy Survey (SNLS Sullivan et al. 2011, and references therein), and the Foundation Survey within Pan-STARRS (e.g, Foley et al. 2017).

In addition to these largely ground based efforts, there are devoted space-based programs, one of note being the SWIFT Supernova Program that is capable of producing early- to latetime UV measurements useful for understanding the local SN Ia environs (Brown et al. 2015a).

A database of SNe discoveries (of all types), largely driven by transient monitoring programs, is maintained on the Latest Supernovae website (Gal-Yam et al. 2013), ${ }^{2}$ which includes discovery imaging and classifications on a day-to-day basis. Individual teams maintain their own databases (both publicly available and proprietary), but the Open Supernova Catalog $^{3}$ provides a uniform access point for both original observations and derived parameters with extensive citations (Guillochon et al. 2016).

\subsection{SN Ia as Cosmological Probes}

The role of SNe Ia in the construction of our concordance cosmology is hard to understate. They provide the largest sample of tracers for measuring the Hubble constant (as demonstrated in Fig. 1) and their intrinsic luminosity gives us access to the evolution of the Hubble constant over time. The latter led to the discovery of the accelerating Universe (Perlmutter et al. 1997, 1998, 1999c; Riess et al. 1998b; Schmidt et al. 1998a). Of particular interest are the proceedings of the Centennial Symposium for the Carnegie Institution of Washington, entitled "Measuring and Modeling the Universe," which carefully reflects on the establishment of the concordance cosmology (Freedman 2004). More recent reviews for which SNe Ia play a central role are: the Hubble constant by Freedman and Madore (2010), for Dark Energy and the accelerating Universe by Frieman et al. (2008), and a prospectus for the long term impact on physics by Goobar and Leibundgut (2011). The hundreds of citations to these review articles indicate the speed at which these fields are evolving.

Indeed, in a span of less than 20 years the 'factor of two controversy' in the value of the Hubble constant, ultimately resolved with the HST Key Project (Freedman et al. 2001a),

\footnotetext{
${ }^{2}$ Available: http://www.rochesterastronomy.org/snimages/.

${ }^{3}$ Available: https://sne.space/.
} 
has been recast as a $3 \sigma$ discrepancy between the values measured from $\mathrm{SNe}$ Ia (e.g., by calibration of Fig. 1; Riess et al. 2011, 2016; Freedman et al. 2012) and that inferred from modeling anisotropies in the cosmic microwave background (most recently, Planck Collaboration 2016).

As is demonstrated by Beaton et al. (2016, their Fig. 1), as the uncertainties in either measurement technique have progressively decreased, the discrepancy between the two techniques has only grown. As described in Freedman (2017), the SN Ia derived cosmology 'is at a crossroads.' Thus, it is meaningful to understand the observational metrics through which we understand and characterize the SN Ia, which are driven by the wealth of data that can be collected in the near-field.

For the purposes of the distance scale, SNe Ia in the local and intermediate redshift regime are of the greatest interest, with measurements made on those samples ultimately informing their use at ever more distant regimes. Those samples used for cosmology $(z>0.3)$ ultimately drive a set of criteria that determine the "suitability" of a local or intermediate SN Ia for direct calibration. These restrictions are motivated by the desire to use the same intrinsic distance indicator in all regimes and, due to observability constraints, many of the fundamental parameters (such as the dispersion within a class of SN Ia sample) are only well-characterized in local or intermediate samples (to be discussed in a later section). The requirements from Riess et al. (2016) are: (i) observations taken on modern CCD detectors, (ii) not highly reddenned $\left(A_{V}<0.5\right)$, (iii) discovered before peak brightness, (iv) have 'typical' spectroscopic designations, and (v) relatively homogeneous photometric data (same filters, etc.).

\subsection{SN 2017cbv: a Recent 'Local' SN Ia}

On March 10, 2017, a transient source, DLT17u, was discovered in the nearby $(D=$ $16 \mathrm{Mpc}$ ), face-on barred spiral galaxy NGC 5643 within the context of the $D<40 \mathrm{Mpc}$ (DLT40) one day cadence supernova search (Tartaglia et al. 2017). The top panels of Fig. 2 compare a pre- and post-discovery image of NGC 5643.

Within 20 hours, a spectrum was obtained for the source that classified it as a SN Ia from the characteristic broad emission lines (Hosseinzadeh et al. 2017). Comparison of the spectrum to a series of templates indicated this object was found at least 2 weeks before maximum light and detailed comparisons to template spectra within the Superfit code (Howell et al. 2005) confirmed this was young SN Ia. Based on this classification, the transient DLT17u was renamed to SN 2017cbv.

Additional careful photometric monitoring of the object began nearly immediately after its discovery (e.g., Coulter et al. 2017, among others) and, due to its brightness (it reached $\sim 11.5 \mathrm{mag}$ in $V$ ), both professional and amateur astronomers have participated in data collection. It is noteworthy that NGC 5643 was home to a SN Ia in 2013, SN 2013aa (Parrent et al. 2013), which was extensively monitored by the Carnegie Supernova Project (CSP Freedman et al. 2009; Hamuy et al. 2006). In contrast to SN 2017cbv, SN 2013aa was only classified 'a few days before maximum (Parrent et al. 2013), which limited follow-up observations by other teams.

In Fig. 3, the -11 day spectrum of SN $2017 \mathrm{cbv}$ is compared to the -1 day spectrum of SN 2013aa. There are marked differences between the spectral evolution over just this 10 day time span, but the broad, often asymmetric, spectral features are characteristic of SNe Ia. The ability to find these events early and begin the follow-up process not only aids in the use of a SN Ia for distance determination, but also in revealing their physical evolution. A detailed guide to optical spectral classification for all SNe is given in Filippenko (1997a) 


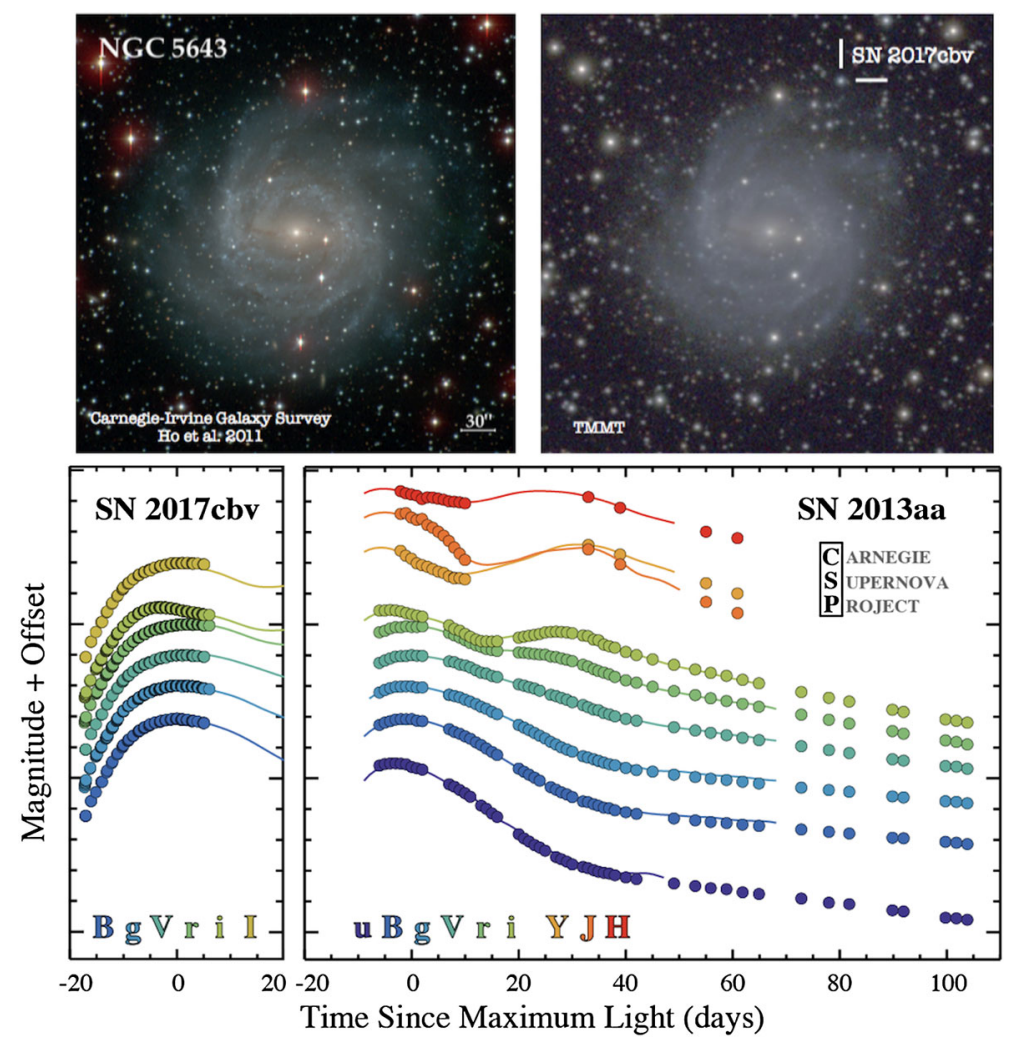

Fig. 2 Imaging data required to characterize SN Ia for use in a Hubble diagram. (top panels) Pre-imaging of NGC 5643 from the Carnegie-Irvine Galaxy Survey (Ho et al. 2011) as compared to a photometric monitoring image of NGC 5643 with SN 2017cbv indicated (imaging using the TMMT; Monson et al. 2017, A. Monson, priv. communication). (bottom panels) Daily photometric monitoring of SN 2017cbv (left) in six photometric bands from a pair of robotic telescopes at Las Campanas Observatory through maximum light. This exquisite pre-maximum photometry is enabled by dual-hemisphere monitoring programs and can be compared light curves from the previous SN Ia in NGC 5643, SN 2013aa (right). The nine-band photometric monitoring from the CSP permits detailed study of the the SN Ia, including direct fits for the extinction law local to the SN Ia. SN 2017cbv will be followed for a similar length of time as SN 2013aa from the ground and space. Small ticks correspond to a luminosity change by 1 mag. Light curves courtesy of A. Monson, M. Seibert, and C. Burns

and for SNe Ia by Parrent et al. (2014); in particular, Parrent et al. (2014) demonstrates the spectral evolution with time, for which the spectrum of SN 2017cbv represents one of the earliest ever observed.

The photometric monitoring data for SN 2017cbv and SN 2013aa are compared in the bottom panels of Fig. 2. Multi-band simultaneous light-curve fits from SNooPy (Burns et al. 2011,2014 ) are also shown for both SNe Ia underneath the individual observations. The key difference is the (i) dense sampling permitted with robotic facilities and (ii) the $>10$ day difference in the starting point of the light curve. Utilizing a SN Ia for a distance measurement requires careful fitting of the time of maximum light, which is less ambiguously determined for SN 2017cbv than for SN 2013aa. Moreover, to simultaneously and self-consistently solve for both the total extinction (the combined foreground Galactic extinction and the internal host-galaxy extinction) and the reddening law (e.g., $R_{V}$ ) local to the $\mathrm{SN}$ Ia, simultaneous 


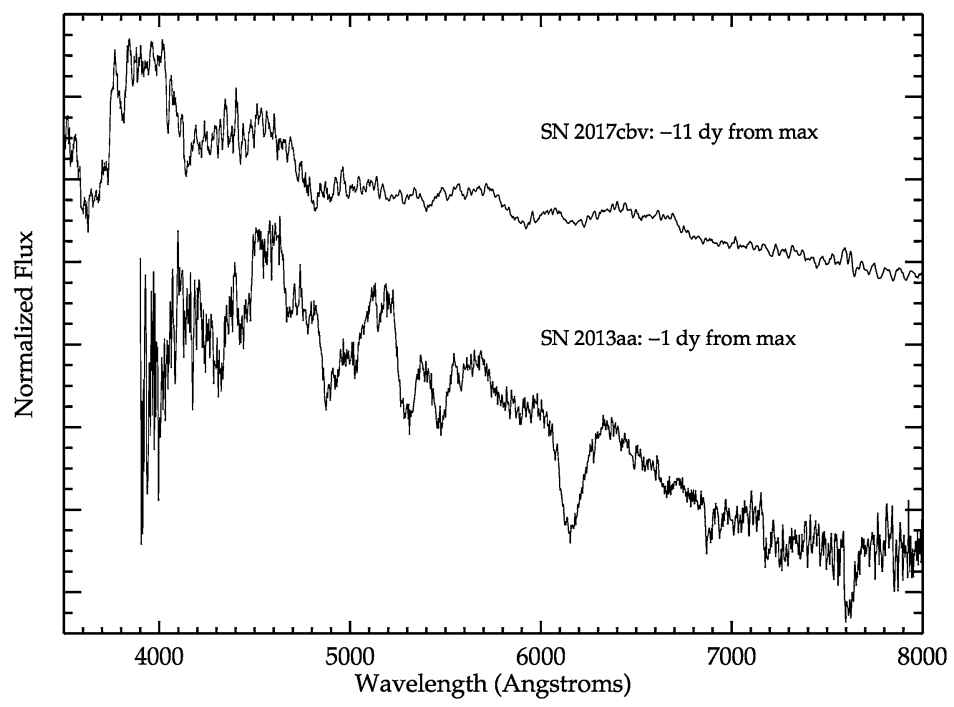

Fig. 3 Classification spectra for the SN Ia in NGC 5643. SN 2017cbv was classified at least - 10 days from maximum and the same day of discovery (top). SN 2013aa was classified -1 day from maximum and three days after its discovery (bottom). These two spectra are demonstrative of the differences in SN Ia spectra on $\sim 10$ day timescales. The spectrum for SN 2017cbv was retrieved from the Transient Name Server (https://wis-tns.weizmann.ac.il/object/2017cbv) and obtained by Hosseinzadeh et al. (2017). The spectrum for SN 2013aa was retrieved via the Latest Supernovae (Gal-Yam et al. 2013) compilation and obtained by Terry Bohlsen (http://users.northnet.com.au/ bohlsen/Nova/sn_2013aa.htm)

multi-band fitting is necessary. Preliminary fitting with SNooPy for both SNe Ia suggest that the total extinction for both objects is consistent with the foreground component; this is consistent with the location of either SN Ia in the outer disk of NGC 5643,

The light curves for SN 2013aa which are typical of the CSP program, show the differences between the light curves observed in blue and red wavelengths. At longer wavelengths (longward of $i^{\prime}$, there is a secondary maximum that occurs around 35 days. The strength and timing of the secondary maximum is correlated with the decline rate (with the fastest decliners showing no bump), which is suggestive that it has some physical connection with the primary maximum (see discussion in Phillips 2012, and their Fig. 1). The near-infrared properties of SNe Ia have revealed additional peculiar classes not apparently in the optical light-curves. For this reason, Phillips (2012) argues that the $Y$ is well positioned to meet the complementary needs of signal-to-noise, insensitivity to dust, well-behaved decline-rate corrections, and simplicity of technology to provide the greatest leverage on the future cosmological measurements using SNe Ia.

The light curves for SN 2013aa also demonstrate the length of time for typical SN Ia follow-up. More specifically, to determine the stretch-factor for the Phillips-relation, $\Delta m_{15}$, observations must be sufficient to (i) determine maximum light and (ii) to follow the light curve beyond 15 days. Both SNe Ia display 'ideal' stretch factors of $\Delta m_{15} \sim 1$ and, thus, both SNe Ia are 'normal' and ideal for use as standard candles. As is demonstrated for SN 2013aa, the 'local' sample is typically followed with a dense cadence for $\sim 40$ days (to detect the secondary maximum) and then a less dense cadence there after for as long as the SN Ia can be observed from the ground. When the SN Ia is no longer detectable from the ground, space-based photometry can be used to put further constraints on progenitor models; SN 2011fe in M 101 has been followed 1840 days post its $B$ maximum (though on months 
long timescales) and observations are planned for at least another 600 days (Shappee et al. 2017).

The discussion of SN 2017cbv and SN 2013aa demonstrates the progress in the level of SN Ia observations in only a few years. Dual-hemisphere transient monitoring enabled by robotic, and largely autonomous, facilities permits early discovery of transient objects. A large-scale networked international community, enabled with state-of-the-art data processing tools, can classify a transient event within a day of its discovery. Classification, in turn, triggers numerous communities poised for appropriate follow-up, which for the use of SN Ia for distances requires photometric monitoring for up to 40 days post-maximum.

\subsection{The 'Local' Sample in Context}

The current limitation of SNe Ia as absolute distance indicators is the calibration of the absolute luminosity of SN Ia. We do not have theoretical predictions of their peak luminosity because there is still continuing uncertainty about their exact nature. Thus they have to be calibrated using other distance indicators, and this is one of the primary purposes for the modern distance ladder. SNe Ia are calibrated by Cepheid stars, using the Leavitt Law, which in turn have to be calibrated to an absolute scale. Currently, the Leavitt law is determined by using both Cepheids in the Milky Way and in the Small and Large Magellanic Clouds. Milky Way Cepheids have distances measured geometrically from their parallaxies, either by Hubble Space Telescope or Hipparcos (van Leeuwen et al. 2007), and new Gaia masurements, for a much larger number of stars (Gea 2017) are now being incorporated into the cosmic ladder. The Leavitt Law in the Large Magellanic Cloud is anchored by eclipsing binaries (e.g. Pietrzyński et al. 2013). The statistical error in the distance measurement of a single Cepheid was estimated to be around 0.3 mag (Riess et al. 2016).

However, as of Riess et al. (2016), there are in total of 19 SNe Ia host galaxies that can be calibrated via Cepheids and the Leavitt Law. Prior to 2016, there were only eight SN Ia calibrators, two of them the same SN Ia used in the HST Key Project (Freedman et al. 2001a). This implies that roughly $\sim 1 \mathrm{SN}$ Ia suitable for the calibration sample is discovered per annum, despite SNe Ia comprising $66 \%$ of the sample of all bright SNe (see Holoien et al. 2017a, and references therein). It is of interest to investigate what is limiting the build-up of the SN Ia calibration sample.

In Fig. 4a, the SNe Ia known from the 'Recent Supernova' database (Gal-Yam et al. $2013)^{4}$ as of March 2016 are binned by the homogenized distance to their host galaxy from NASA Extragalactic Database (NED; Steer et al. 2017). This sample has been cleaned of SN Ia sub-types, but not by extinction (host galaxy or Galactic foreground) or decline rate. The average number of SNe Ia within a single $5 \mathrm{Mpc}$ bin is $\sim 20 \mathrm{SNe}$ Ia, which suggests there are $5 \times$ more $\mathrm{SNe}$ Ia within the volume accessible to HST Cepheid measurements than are currently in the calibration sample (using $\sim 35-40 \mathrm{Mpc}$ as the limiting volume).

With the dramatic increase in efficiency for transient follow-up and classification described above, is it not unreasonable to attribute this large number entirely to recent discoveries. In Fig. 4b the $95 \mathrm{SNe}$ Ia within $40 \mathrm{Mpc}$ are binned by their discovery year from 1995 to 2016. For reference, the ASAS-SN all-sky project has been in operation since 2013 (Holoien et al. 2017d), but only reached peak efficiency in the latter half of 2015 (Holoien et al. 2017a), shortly before this data were compiled. From inspection of Figure 4b, the number of SNe Ia discovered per annum fluctuates widely, but, in general, the numbers do not trend strongly with the onset of all-sky monitoring programs (albeit these programs

\footnotetext{
${ }^{4}$ Available http://www.rochesterastronomy.org/supernova.html.
} 
Fig. 4 'Local' SNe Ia demographics as of as of March 01, 2016. (a) Number of normal SNe Ia discovered since 1995 as a function of the NED

Homogenized distance for their host. Error bars show the Poisson uncertainty for each bin. While there are few SNe Ia within $20 \mathrm{Mpc}$, the distribution beyond $20 \mathrm{Mpc}$ is relatively flat. (b) Number of normal SNe Ia discovered per year since 1995 within $40 \mathrm{Mpc}$. There are a total of $95 \mathrm{SN}$ Ia using these distances. This suggests that if primary distance techniques can push into this volume, a vastly larger number of SNe Ia can be independently calibrated to study the known biases in the $\mathrm{SNe}$ Ia population
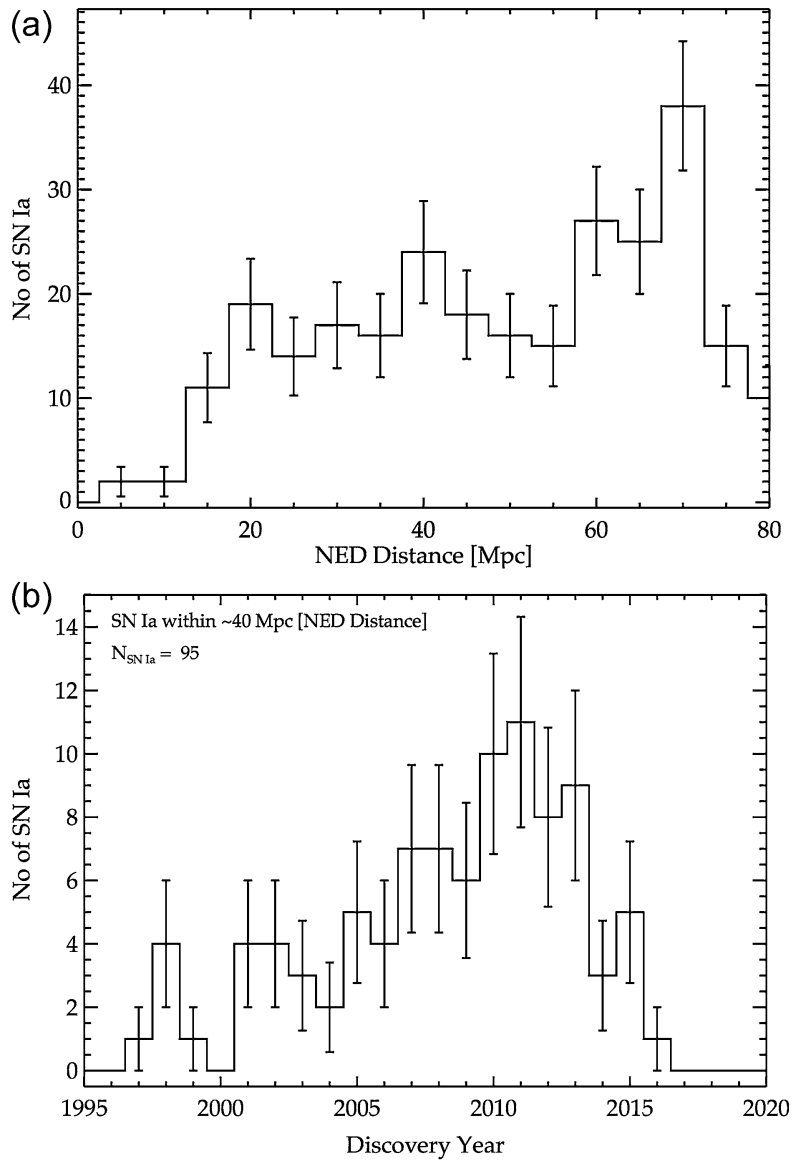

permit unprecedented early detection; e.g., Fig. 2). Analyses of yearly rates over the next few years, however, will be more informative with programs like ASAS-SN having become dramatically more efficient (e.g., Holoien et al. 2017b).

Of the $95 \mathrm{SN}$ Ia within $40 \mathrm{Mpc}$, nearly half $(\sim 40)$ have sufficient photometric data sufficient to be included in the calibration sample (e.g., similar in spirit to Fig. 2; C. Burns and B. Shappee, priv. communication). Despite having sufficient data to determine a SN Iabased distance, these host galaxies lack robust independently determined distances from the Cepheid-based distance ladder. The reason for the lack of independent distances is the unsuitability of the host galaxy for Cepheid based distances, either due to its star formation history, an edge-on inclination, or its morphological type. Thus, utilization of a distance ladder constructed using a standard candle that can be applied to edge-on and non-star forming galaxies has the potential to dramatically improve the size of the calibration sample. Such a distance ladder is explored using old stellar populations in Beaton et al. (2016), and these distance methods are described elsewhere in this volume. As discussed in the following section, expanding the calibration sample to better reflect the demographics of SNe Ia in the larger, Hubble flow sample is an important step for further refinement of SNe Ia derived cosmological parameters. 
In principle, the use of multiple calibrators for a single galaxy should help to control the systematic errors. For example, the use of galaxies where both SN I and II have been detected would be valuable, but unfortunately, there are only a few of such galaxies, and they lack distance determination based on the Tip of the Red Giant Branch (TRGB) and/or Cepheids. Probably, with the new facilities the number of galaxies with both SN types and a good distance determination will increase.

Apart from Cepheids, NGC 4258, an active galaxy with spatially resolved water maser emission is used as additional distance indicator since this galaxy is also a host for numerous Cepheids. A combination of four geometrical distance calibrators (Riess et al. 2016) allowed a determination of $H_{0}$ with $2.4 \%$ uncertainty, and this is the uncertainty currently underlying all cosmological measurements done with the use of SNe Ia as a systematic error.

\subsection{In the Hubble Flow}

Our discussion of the 'local' SNe Ia in NGC 5643 is characteristic of the attention received by these rare 'local' calibrators. The dramatically larger sample of SNe Ia in the Hubble Flow receive less attention in their data collection, but represent a much more complete sample from which to draw inferences on SN Ia as a population. More specifically, these are usually only monitored for $\sim 20$ days to obtain decline rates and may not have extensive multi-wavelength data or time-resolved spectroscopy.

There are three main sources of systematic uncertainty that hinder the use of SNe Ia and our understanding of the physical phenomena inferred from them (adapted from Hicken et al. 2009):

1. Photometric accuracy: large samples of SN Ia light curves are a largely heterogeneous ensemble of photometry coming from multiple programs operating with different skyfootprints and detectors.

2. Host-galaxy reddening: SN Ia are discovered in galaxies with a range of host morphologies and are found across these galaxies and thus span a large range of local conditions. Many SN Ia show anomalous $R_{\lambda}$ extinction parameter, where $R_{\lambda}=A_{\lambda} / E_{B-V}$ is defined by the ratio of the total extinction to the color excess, but this could be attributed to many factors. Constraining the host-galaxy reddening is of paramount importance.

3. SN Ia population differences and/or evolution: the intrinsic variation of the SN Ia population as a function of the stellar populations of the host galaxy, etc.

The large scale programs described earlier are all aimed at addressing item (1). Item (2) will be discussed in detail later in this volume (see Sect. 2.8). The relevant results for item (3) are summarized in the points to follow.

Multi-SN Ia Systems There are only two current 'local' galaxies that are host to multiple SN Ia in the modern observation era. These are NGC 1316, a massive elliptical galaxy in the Fornax cluster, and NGC 5643, a spiral galaxy (discussed above). Stritzinger et al. (2010) used the four events in NGC 1316 and were able to determine that the three normal SNe Ia provided consistent distances at the 5\% level and demonstrated that the fourth SN Ia, a fastdecliner, was discrepant at the 25-30\% level. The two SNe Ia in NGC 5643 (Figs. 2 and 3) will be the first time a test of this nature can be performed on a late-type host. Moreover, both of these galaxies are sufficiently local that distances can be derived independent of $\mathrm{SNe}$ Ia to extend such tests in absolute terms (in lieu of differential analyses). 
Intrinsic Scatter Using early data from the CSP, Folatelli et al. (2010) studied the scatter of the best studied SNe Ia in their sample; this set included $23 \mathrm{SNe}$ Ia with multi-wavelength data similar to that presented for SN 2013aa in Fig. 2. A scatter of $0.11 \mathrm{mag}(5 \%)$ in distance was reported. Folatelli et al. (2010) noted that the dispersion increased with decreasing distance, which implies that much of the observed dispersion could be accounted for with peculiar velocities. They discussed additional reasons for the observed trends, concluding that many observational and (potential) intrinsic effects could contribute to the scatter. A deeper analysis using a larger set of homogeneous data would be beneficial to understand the intrinsic scatter in SNe Ia magnitudes.

Host Mass Bias Sullivan et al. (2010) find a strong dependence on the mass of the host. Massive galaxies are systematically brighter by 0.06 to 0.09 mag (3\% to $5 \%$ in distance; $>3 \sigma$ difference). Such effects will average out for cosmology if the distribution of host galaxy masses remains relatively constant in time, but it is unlikely that this is the case for distant SNe Ia samples.

Local Star Formation Bias Rigault (2013) found a dependence of derived SNe Ia magnitudes as a function of the local $(1 \mathrm{kpc})$ environment around the $\mathrm{SN}$ Ia as probed by $\mathrm{H} \alpha$ surface brightness. SN Ia in passive (i.e., non-star forming) environments were found to be brighter by $0.094 \pm 0.031 \mathrm{mag}$ ( $5 \%$ in distance), which is termed the local star formation bias. Rigault et al. (2015) revisited this issue using both an independent sample of SN Ia (that of Hicken et al. 2009) and using FUV maps to trace the local region. Rigault et al. (2015) find that SN Ia in locally star-forming environments are $0.094 \pm 0.025 \mathrm{mag}$ (5\% in distance) fainter; a $3.8 \sigma$ difference. If the Hicken et al. (2009) sample is isolated to just the most massive hosts, nearly $50 \%$ of the SN Ia arise from locally passive environments. The local SN Ia calibration sample is almost entirely star-forming (Rigault et al. 2015; Riess et al. 2016). On the contrary, Jones et al. (2015) investigated this issue with a larger sample and found no evidence of a significant local star formation bias.

These effects are complicated and may bias attempts to better understand which galaxies produce SN Ia. More complex modeling as in Holoien et al. (2017c) could be of use to disentangle these effects in the general SN Ia population as well as the continued largescale study of detailed SN Ia host demographics (e.g., Holoien et al. 2017b, and references therein).

Additional systematic error in SNe Ia measurements may come from weak lensing effect, particularly for the high redshift sources, above $z=1.5$. Weak lensing causes additional dispersion in the measured luminosity distances, and the distances are on average effectively larger. Hilbert et al. (2011) estimated that this effect introduces systematic error of about $2 \%$ at redshift 1.5, but the appropriate simulations may allow to reduce this effect. This applies also to other measurement methods based on standard candle approach.

\subsection{Progenitors}

Despite their use as cosmological standard candles and their relevance also for galactic chemical evolution, consensus about the nature of SN Ia progenitor systems is still lacking, and this may affect their use as the tracers of the Universe expansion.

Any model for SN Ia progenitors must satisfy a number of observational constraints. The first obvious ones are the lack of hydrogen $\left(\Delta M_{H}<0.01 M_{\odot}\right)$ and helium in the spectra, the range of values of the peak luminosity and their correlation with the light curve width, and a total kinetic energy of the ejecta of $\sim 10^{51} \mathrm{erg}$. Our understanding of SN Ia 
lightcurves dictates that the peak luminosity is determined by the mass of radioactive ${ }^{56} \mathrm{Ni}$, whilst the lightcurve width depend on the opacity, determined by iron-group elements like ${ }^{56} \mathrm{Ni},{ }^{58} \mathrm{Ni},{ }^{54} \mathrm{Fe}$. These latter two elements are non-radioactive and do not contribute to the peak luminosity. The temporal evolution of the spectra also allows to reconstruct the radial composition of the ejecta. All these observations taken together imply the presence of $\sim 0.1-1.0 M_{\odot}$ of ${ }^{56} \mathrm{Ni}$ in the ejecta (but very little in the outer layers), $\sim 0.2-0.4 M_{\odot}$ of $\mathrm{Si}$, $\mathrm{S}, \mathrm{Ca}, \mathrm{Ar}$, and less than $0.1 M_{\odot}$ of $n$-rich isotopes $\left({ }^{54} \mathrm{Fe},{ }^{58} \mathrm{Ni}\right)$.

It was recognized very early on Hoyle and Fowler (1960) that SN Ia must be thermonuclear explosions in electron degenerate matter, pointing to exploding white dwarfs (WDs) as the cause of SN Ia events. Given that single WDs form with masses below the Chandrasekhar mass and are intrinsically stable objects, their evolution must be perturbed by a companion in order to cause the required explosion. Irrespective of the companion and the trigger mechanism, one can firstly investigate which WD mass+explosion mechanism can be invoked to explain 'normal' SN Ia observations (see, e.g., the review by Röpke et al. 2011). In terms of WD masses, one can divide the potential progenitors into two broad classes, namely Chandrasekhar and sub-Chandrasekhar mass progenitors.

In the Chandrasekhar mass scenario a $\mathrm{C}-\mathrm{O}$ WD in a binary system approaches the Chandrasekhar mass due to accretion from the companion, and C-burning reactions set in at the centre when the density is of the order of $10^{9} \mathrm{~g} \mathrm{~cm}^{3}$. After a non-explosive initial 'simmering' phase (see, i.e., Lesaffre et al. 2006) lasting 1000-10000 yr, that burns only a small amount of $\mathrm{C}$, a thermonuclear runaway starts. The number of ignition sparks of the burning front and their distribution are unknown and have a significant impact on the characteristics of the explosion. The best agreement with observations is achieved considering a combustion front propagating at first subsonically (deflagration) then supersonically (detonation-Blinnikov and Khokhlov 1986). This so-called 'delayed detonation' allows the production of a layer of intermediate-mass elements that encompasses almost all of the outer ejecta, as required by observations. The transition from deflagration to detonation is typically parametrized and not derived from first principles. Variations of the flame ignition configuration and transition to detonation can reproduce at least qualitatively the observed luminosity-decline rate relation (Kasen et al. 2009).

In the sub-Chandrasekhar mass scenario (see, i.e., Woosley and Weaver 1994) the helium layer that surrounds the $\mathrm{CO}$ core increases its mass due to accretion from the binary companion. When the mass of the He-layer reaches a critical value, a detonation is triggered by compression. This detonation burns the He-layer and drives a shock wave into the core, triggering a detonation of carbon and the explosion of the WD. In this scenario, a variation of the exploding WD mass could potentially explain the luminosity-decline rate relation. There are however problems related to the explosive nucleosynthesis, for not enough stable $\mathrm{Fe}$ and $\mathrm{Ni}$ isotopes to match late time spectra are produced (because of too low densities at explosion), and too much ${ }^{56} \mathrm{Ni}$ is made in the He-shell ashes (Röpke et al. 2011). If the mass of the He-layer that detonates is $\sim 0.01 M_{\odot}$, as possible for WDs around $1 M_{\odot}$ (Bildsten et al. 2007), negligible ${ }^{56} \mathrm{Ni}$ seems to be produced, although other calculations do not confirm this result (Röpke et al. 2011).

Both these WD mass+explosion mechanisms put forward to explain SN Ia events need the presence of a companion in a binary system. We can traditionally divide the progenitor systems into two broad classes, namely single-degenerate (SD) and double-degenerate (DD) systems.

In a typical SD system (Whelan and Iben 1973) the WD accretes $\mathrm{H}$ from a non electrondegenerate companion. Hydrogen is burned to He first, then to C, and the WD mass increases to the Chandrasekhar limit. As shown by Nomoto and Kondo (1991), stable H-burning is 
possible only for a finely tuned accretion rate of around $3 \times 10^{-7} M_{\odot} \mathrm{yr}^{-1}$. Lower rates $\left(<10^{-8} M_{\odot} \mathrm{yr}^{-1}\right)$ produce a Nova event, which expels the ashes of the accreted material and possibly even some of the original WD material (e.g. Yaron et al. 2005). This may not be the case at intermediate rates, where recurrent Novae systems retain some of the accreted mass (e.g. Hachisu and Kato 2000). If the accretion rate is too large, the WD is expected to expand to giant-like dimensions, and engulf the donor star in a common envelope which inhibits further accretion onto the WD. Hachisu et al. (1996) have proposed that an optically thick wind emerges from the WD that stabilizes the mass transfer. In this way the mass accretion may continue, but at a reduced rate. Generally, there is only a narrow range of accretion rates in which the WD can retain the matter and grow in mass.

If the non-degenerate companion is a He-star (produced by a previous common envelope episode) the WD can accrete He leading to either a Chandrasekhar or a sub-Chandrasekhar explosion (see, i.e., Woosley and Kasen 2011; Piersanti et al. 2014), depending on the accretion rate. Accretion rates of the order of $\approx 10^{-8}-10^{-9} M_{\odot} \mathrm{yr}^{-1}$ lead to a He-detonation and a sub-Chandrasekhar explosion; in case of rates of the order of $\approx 10^{-6} M_{\odot} \mathrm{yr}^{-1}$ steady He-burning on the WD surface can produce a Chandrasekhar mass WD that ignites $\mathrm{C}$ in the core.

In a DD system (Tutukov and Yungelson 1981) two WDs orbit each other and the system loses energy through gravitational waves emission. As the two components come closer and closer, eventually the least massive object fills its Roche-lobe and mass transfer sets in, with the more massive WD accreting $\mathrm{C}-\mathrm{O}$ from an accretion disk/torus. If this WD can reach the Chandrasekhar mass a delayed-detonation will ensue. It is however uncertain whether this happens; even in case of a system where the combined mass of the two WDs exceeds the Chandrasekhar limit, an off-centre carbon deflagration followed by conversion to an $\mathrm{O}-\mathrm{Ne}-\mathrm{Mg}$ WD seems to be a likely outcome. Nearing the Chandrasekhar mass the $\mathrm{O}-\mathrm{Ne}-$ $\mathrm{Mg}$ WD undergoes an electron induced collapse that leads to the formation of a neutron star. An alternative path that could lead to a SN Ia explosion is a so-called violent merger (see, i.e., Pakmor et al. 2010). At the merger, an accretion stream can dynamically produce a high temperature when hitting the WD surface. If high enough, this leads to the WD explosion according to the sub-Chandrasekhar double-detonation scenario. This scenario seems however efficient only for systems whose primary WDs are massive $\left(>0.8-0.9 M_{\odot}\right)$ and the mass ratio is high $>0.8$. Pakmor et al. (2013) find that the presence of helium facilitates the ignition.

Despite the enormous efforts of the astronomical community, there has not yet been a convincing identification of a SN Ia progenitor (Li et al. 2011a; Nielsen et al. 2012). Furthermore, none of the proposed formation channels are clear matches to the observational constraints. Due to the uniformity and continuity of the observed SN Ia properties, there has been a long-time focus to find a single formation channel to explain all properties. However, from the diversity and correlations among the spectral properties of $\mathrm{SNe}$ Ia and their host galaxies, it is also possible that some combination of formation channels is operating.

An advantage to the SD channel is that it naturally explains the uniformity in luminosities as all WDs explode at the Chandrasekhar mass in this channel. On the other hand, the masses of the merged remnants in the DD channel display a broader range $\left(\sim 1.4-2.0 M_{\odot}\right)$. It is usually assumed that the DD systems with super-Chandrasekhar masses are responsible for the super-luminous SN Ia. Another expected signature of the SD channel, is that the companion is expected to survive the explosion and with an anomalous velocity, rotation, spectrum or composition. No such object has been identified unambiguously, for example in Tycho's SN remnant (Ruiz-Lapuente et al. 2004; Kerzendorf et al. 2009; Schaefer and Pagnotta 2012). Also, any mixing of the SN ejecta with that of the companion star during 
the explosion, has not been observed conclusively (Leonard 2007; García-Senz et al. 2012). In a minority of cases, variable $\mathrm{NaD}$ absorption has been detected in the $\mathrm{SN}$ spectra, which has been interpreted as circumstellar material from the companion in the SD channel (Patat et al. 2007; Sternberg et al. 2011). This signature, however, can also arise in the DD channel from a post-merger, pre-explosion wind (Shen et al. 2012). Furthermore, one expects soft $\mathrm{X}$-ray emission from SD systems produced by the steady H-burning on the WD surface, but not enough emission has been observed from resolved nor unresolved sources (Gilfanov and Á Bogdán 2010; Di Stefano 2010). The emission might be shielded within optically thick outflows and reprocessed to UV-emission (Hachisu et al. 2010; Nielsen et al. 2013; Wheeler and Pooley 2013), but so far a population of these sources has not yet been detected (Woods and Gilfanov 2013; Lepo and van Kerkwijk 2013). Lastly, SN2011fe is a recent and close SN Ia that is well observed in optical, radio and X-ray wavelengths. These observations have ruled out most types of donor stars in the SD channel, and therefore favor a DD progenitor (e.g. Chomiuk 2013); see also Maoz et al. (2014) for a review on the observational clues to the SN Ia progenitor problem.

Additional constraints on the SN Ia progenitor channels are provided by the observed delay-time-distribution (DTD) of SN Ia events. The DTD is defined as the time interval between a star formation episode and the explosion of the related supernovae. It illustrates the distribution in evolutionary timescales of the SN Ia progenitor, which vary for the different progenitor scenarios. The DTD has been studied observationally in a range of surveys, environments and redshifts, and the emerging picture is remarkably coherent (for a review, see Maoz and Mannucci 2012). Type Ia SNe occur in young and in old stellar populations, reaching delays as long as a Hubble time. The SN Ia rate peaks at short delays of $<1 \mathrm{Gyr}$ and declines at longer delay times. The DTD is best described by a power-law with an index of about -1 for delays of $1<t<10 \mathrm{Gyr}$. At shorter delays, there is still some uncertainty regarding the precise shape of the DTD. Despite the consistency in the shape of the DTD from a variety of methods, the normalization or time-integrated DTD shows variations between rates in different galaxy types. SN Ia rates in galaxy clusters are found to be a factor $\sim 5$ higher than rates based on volumetric galaxy samples. Further research is needed to test if this is due to differences in the methods and samples, or if there is indeed an enhancement of the SN Ia rate in cluster environments (e.g. Maoz et al. 2012; Maoz and Graur 2017).

The DTDs for different progenitor channels have been estimated by analytical approaches and by modeling the evolution of binary populations (see Wang and Han 2012, for a review). For the double degenerate channel, there is a good agreement on the DTDs (e.g. Nelemans et al. 2013). The DD model gives rise to delay times ranging from a few Myr up to a Hubble time. Within these delay times, the DTD shape is a continuous power-law with a slope of roughly -1 , which is remarkably similar to the observed DTD. The timeintegrated DTD from the DD channel is compatible with the lower limit of the observed rate. The DTD from double WDs that undergo a violent merger is similar to that of the classical DD channel (Ruiter et al. 2013).

For the single degenerate channel, there is a large diversity in the predicted SN Ia rates spanning over several orders of magnitude (e.g. Nelemans et al. 2013). Differences arise due to ill-constrained aspects of binary formation and evolution, most importantly the accretion efficiency of WDs (Bours et al. 2013) and common-envelope evolution (e.g. Claeys et al. 2014, see Ivanova et al. 2013 for a review). The predicted rates of the SD channel tend to be (far) below that of the DD channel and the observed rate. Typically, the delay times from the SD channel range from a few-hundred Myr to a few Gyr. The models show that SD DTDs have a sharp drop after a few Gyr, in contradiction with the observed DTD. The drop can be understood by the limited range of donor masses $\left(\sim 2-3 M_{\odot}\right)$ that transfer matter to the WD at the necessary rates to ensue WD mass growth. 
If the donor star is a He-star, the delay times are very short i.e. 50-200 Myr (Wang et al. 2009), due to the fact that He-stars evolve from more massive main-sequence stars with shorter evolutionary timescales than in the standard SD channel.

An adaptation to the SD channel has been proposed that resolved some of the observationally discrepant signatures. In the so-called 'spin-up/spin-down'-model (Justham 2011; Di Stefano et al. 2011), WD rotation due to accretion onto the WD is invoked to support against the collapse and ignition of the WD. The time that it takes for the WD to spin down and explode leads to a prolonged delay time. This may solve the issue that the classical SD progenitors do not have long delay times that are observed. Furthermore, if the timescale for spin down is sufficiently long, any traces of the mass transfer and even the companion could disappear. Lastly, the 'spin-up/spin-down'-model also allows for the possibility of super-Chandrasekhar SN Ia.

\subsection{Extinction/Color Corrections for Type-Ia Supernovae}

A common perception exists that the dominant parameter driving the standardization of $\mathrm{SNe}$ Ia for measuring distances is related to the decline-rate or light-curve shape (Phillips 1993; Riess et al. 1995; Hamuy et al. 1996; Perlmutter et al. 1997). However, corrections based on the color of the SN Ia are at least as important (Riess et al. 1996; Phillips et al. 1999). A variety of approaches has been used for this correction, and these differences ultimately relate to the cause of color variations in $\mathrm{SN}$ Ia, something that has been difficult to pin down.

All SN Ia luminosity distance measurements must correct for extinction by dust in the Milky Way, typically using the dust maps of Schlegel et al. (1998), or more recently, Schlafly and Finkbeiner (2011), to provide an estimate of the reddening $E(B-V)_{\mathrm{MW}}$ along the line of sight. This reddening is converted into an extinction in the observed passbands using the dust law of Cardelli et al. (1989), with updates from O'Donnell (1994) and Fitzpatrick and Massa (2007), typically assuming an extinction law parameter $R_{V} \approx 3.1$ as found in the diffuse ISM. Care must be taken to account for the time-evolving spectral energy distribution (SED) of the supernova. Uncertainties in the Milky Way extinction correction are correlated across all observations of an individual supernova, and systematic uncertainties in the Milky Way extinction correction can have important effects on parameter inferences from a supernova sample. Nonetheless, hereafter we discuss SN Ia extinction or color corrections under the assumption that the Milky Way component has been properly removed.

In analogy to Milky Way extinction, the light from distant SN Ia is extinguished by dust in the supernova host galaxy (which acts on the rest-frame light in contrast to the Milky Way extinction which acts after the light has been redshifted to the observer frame). One approach to this correction is to assume that the intrinsic colors (or SED) of a SN Ia are strictly determined by its light curve shape, i.e. that SN Ia are intrinsically a one-parameter family. Color variation among SN Ia is then ascribed to intrinsic stochasticity (random scatter around the nominal color) and extrinsic reddening by host-galaxy dust. This is the approach used by the Multicolor Light Curve Shape method (Riess et al. 1996, 1998b) and as extended to MLCS2k2 (Jha et al. 2007), as well as adaptations of the $\Delta m_{15}$ method (Phillips et al. 1999; Burns et al. 2011). Even if the intrinsic SN Ia light curves are described with a more complex parameterization, extrinsic host-galaxy extinction can be treated separately, as in the BayeSN methodology (Mandel et al. 2011).

Applying this method gives a surprise: Hubble diagram residuals are minimized when the ratio between the inferred extinction $A_{V}$ and color excess $E(B-V)$ has a value $R_{V} \approx$ $1-2$, significantly lower than the canonical $R_{V} \approx 3.1$ (e.g., Conley et al. 2007). This could 
be interpreted as saying the typical dust in SN Ia host galaxies has smaller grains on average than Milky Way dust, with significantly more reddening for a given amount of extinction.

Indeed, we find strong evidence for "weird" low- $R_{V}$ dust in heavily extinguished SN Ia (e.g., host reddening $E(B-V) \geq 1 \mathrm{mag}$ ). This has been inferred using near-UV through near-infrared observations of reddened objects like SN 1999cl, 2002cv, 2003cg, 2006X, and SN 2014J (Elias-Rosa et al. 2006, 2008; Krisciunas et al. 2006, 2007; Wang et al. 2008b; Burns et al. 2014; Amanullah et al. 2014, 2015; Foley et al. 2014; Brown et al. 2015b; Gao et al. 2015). Independent, corroborating evidence comes from linear polarization measurements; dust scattering imprints a wavelength dependence to the continuum polarization fraction that reflects the grain size distribution and $R_{V}$. For several of these heavily-reddened objects, the wavelength of the continuum polarization peak implies $R_{V} \lesssim 2$ (Kawabata et al. 2014; Patat et al. 2015).

An intriguing possibility is that this strange dust is located near the supernova, in the circumstellar environment of the progenitor system, perhaps with multiple scattering playing a role (Wang 2005; Goobar 2008; Foley et al. 2014). This could help explain why such dust is not evidently seen in the Milky Way ISM, for example. Indeed, some of these heavilyreddened SN Ia show time-variable absorption lines (Na I D, K I) in high-resolution spectra, interpreted as arising from circumstellar gas interacting with the $\mathrm{SN}$ radiation field (Patat et al. 2007; Blondin et al. 2009; Graham et al. 2015).

However, the preponderance of the evidence suggests this low- $R_{V}$ dust is interstellar. Many of these SN Ia show light echoes, with dust sheets $>10 \mathrm{pc}$ away from the supernova (i.e., not circumstellar Wang et al. 2008a; Crotts and Yourdon 2008; Crotts 2015; Maeda et al. 2015; Yang et al. 2017). Moreover, the dust seems to correlate best with diffuse interstellar bands (DIBs) rather than circumstellar gas absorption (Phillips et al. 2013) and moreover, for SN 2014J most of the gas absorption originates on interstellar, not circumstellar scales (Ritchey et al. 2015; Jack et al. 2015; Maeda et al. 2016).

Because of the difficulties in arriving at a physical understanding of the relationship between the observed luminosity and color of SN Ia, a leading approach is to make this an entirely empirical correction, analogous to the light curve shape correction. Tripp and Branch (1999) suggested a simple two-parameter empirical correction, with the SN Ia $B$ band luminosity linearly regressed against a light curve shape parameter and a color parameter. The SALT/SALT2 (Guy et al. 2005, 2007) and SiFTO (Conley et al. 2008) light curve fitters adopt this model. The single color coefficient (called $\beta$ in these models) is again found to be significantly lower $(\beta \approx 2.5)$ than what would be expected by standard dust $\left(R_{V}=3.1 \leftrightarrow \beta=4.1\right)$.

These fitters are aimed primarily at cosmological SN Ia samples, i.e. objects typically with low extinction, not the heavily reddened SN Ia for which there is more direct evidence of unusual dust. Thus, it is not clear whether the low values of $\beta$ in cosmological samples arise from the same kind of unusual dust (just less of it). For example, measurement uncertainties would lead to color "noise" that was uncorrelated to SN luminosity; if this noise level were comparable to the dust reddening (typically $E(B-V) \leq 0.3$ mag for these "cosmological" objects), the effective (observed) value of $\beta$ would be lowered (della Valle and Panagia 1992). Similarly, if the SN Ia had intrinsic color variations, perhaps also correlated to luminosity with some $\beta_{\text {intrinsic }}$, the effective $\beta$ would be intermediate compared to $\beta_{\text {dust }}$ (Mandel et al. 2016).

There is positive evidence that intrinsic color variations and the error model for SN Ia colors may be playing a role. By using spectroscopic indicators that are independent of color (line equivalent widths or velocities), it is possible to ascribe an intrinsic color to an individual SN Ia, and thus determine the color excess (observed minus intrinsic). Correlating 
the SN Ia luminosity against this color excess (rather than the observed color) yields an extinction law consistent with normal dust $\left(R_{V} \simeq 3 ; \beta \simeq 4\right.$ Chotard et al. 2011; Foley and Kasen 2011; Foley et al. 2011; Sasdelli et al. 2016) for low-extinction objects. Similarly, an intrinsic variation "color-smearing" model, combined with a normal dust law, can explain the luminosity-color relation and its scatter better than a single linear model can (Scolnic et al. 2014; Mandel et al. 2016). Nonetheless, strange (strongly-reddening, low- $R_{V}$ ) dust is still necessary for highly-reddened SNe Ia (Mandel et al. 2011).

The emerging picture to explain the relationship between SN Ia luminosity and color has grown quite complex, including intrinsic color variations that may depend on light-curve shape, spectral features, or even host galaxy environment, normal Milky Way-like dust (in the SN host galaxy and in the Milky Way), and strange, likely interstellar but possibly also circumstellar, low- $R_{V}$ host-galaxy dust. Achieving the most precise and accurate distances from SN Ia will require modeling and disentangling all of these effects, and perhaps more. These effects are likely to be more severe for high-redshift SN Ia science, for which there might be significant evolution relative to the Hubble-flow sample, and less severe in measuring $H_{0}$ from $\mathrm{SNe}$ Ia, where the calibrator sample is better-matched. For example, using SNe Ia as near-infrared standard candles, less sensitive to extinction corrections than in the optical, does not significantly changed the derived value of $H_{0}$ (Dhawan et al. 2017).

\subsection{Summary}

Future discoveries with SN Ia cosmology are now built on a framework of large-scale, homogeneous follow-up that unite teams that (i) find, (ii) classify, and then (iii) characterize the SN Ia. There are differences in the follow-up for the 'local' sample of SN Ia, which are ultimately used to set the SN Ia absolute luminosity, those SN Ia discovered in the Hubble Flow (to $z \sim 0.1$ ), which are used to determine the Hubble constant, and those found at higher redshifts, which are used for understanding the acceleration of the Universe. The low-redshift samples, however, remain the lens for understanding the physics of SN Ia and projecting that back in time; of key interest being how SN Ia properties may evolve with the decreasing progenitor metallicity over cosmic time and the apparent star-formation biases. Thus, further development of the 'local' sample and bolstering of the low-redshift sample remains as critical as the original questions posed by Shapley (1919) as to the size of the Universe.

\section{Supernovae II}

Type IIP SNe can be used as "standardized" candles to estimate distances well within the Hubble flow, with a rms precision of the order of 10-15\%. This occurence makes them interesting cosmological distance indicators, since they are produced by different stellar populations than type Ia SNe, making them useful sanity check of the type Ia SNe-based results. More generally, it has demonstrated that all type II (IIP + IIL) SNe can be used as standardized candles (e.g., de Jaeger et al. 2015, 2017). Here the current status of the type IIP SNe standardized candle method is reviewed, pointing out the need for a calibration based on primary distance indicators.

\subsection{Introduction}

In the classical classification scheme (Filippenko 1997b), type IIP SNe are characterized spectroscopically by strong hydrogen emission features, with strong P-Cygni profiles; and 
Fig. 5 Absolute V-band magnitudes of a sample of 60 type IIP SNe, as published in Anderson et al. (2014).

Reproduced by kind permission of the authors

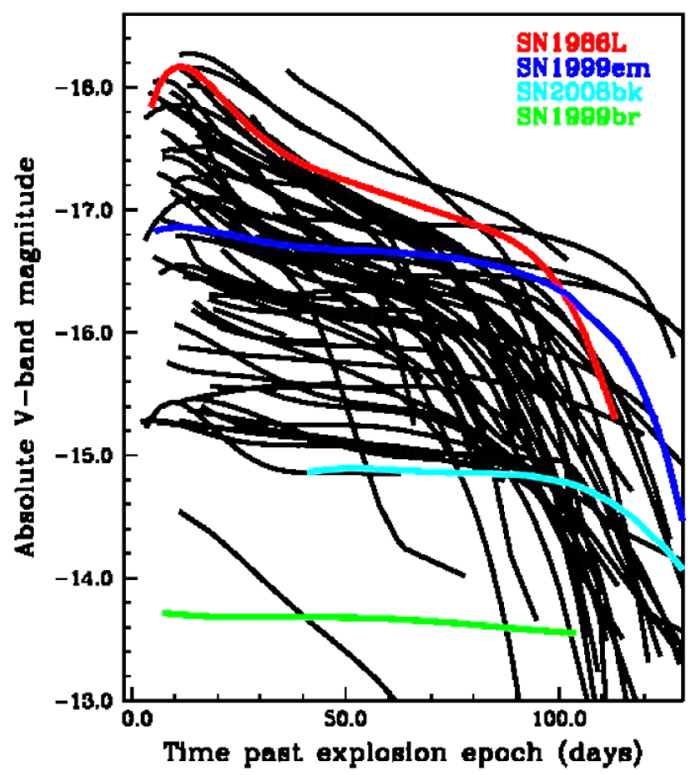

photometrically by a long plateau, lasting on average $\sim 80$ days, followed by a sudden drop in luminosity and a subsequent settlement on the radioactive tail.

From the physical point of view, both theoretical (e.g., Grassberg et al. 1971; Litvinova and Nadezhin 1983; Utrobin and Chugai 2008; Pumo and Zampieri 2011) and empirical investigations (e.g., Smartt et al. 2009) show that type IIP SNe are the product of a core collapse of a small to moderate massive progenitors, typically red supergiants (RSG). Interestingly, while on the basis of the empirical models (Heger et al. 2003; Walmswell and Eldridge 2012) we expect that type IIP SNe are the final fate of progenitors of masses between $8 M_{\odot}$ and $30 M_{\odot}$, empirical evidence found progenitors only in the range $8-17 M_{\odot}$. This discrepancy has been dubbed the "RSG problem" (Smartt 2009). However, some claims in the recent literature suggest higher mass limits (e.g., Dall'Ora et al. 2014), while independent studies of the massive star populations in the Local Group found RSGs with masses up to $\sim 25 M_{\odot}$ (Massey et al. 2000, 2001).

The characteristic photometric plateau is supported by the hydrogen recombination front, which recedes in mass as the photosphere expands and cools down, producing a constant luminosity. As the density lowers down to values in correspondence of which the atmosphere becomes transparent, a sudden drop ( $~ 30$ days) in luminosity of several magnitudes is observed, and the light curve is subsequently powered only by the radioactive decay of ${ }^{56} \mathrm{Co}$ to ${ }^{56} \mathrm{Fe}$. In this phase, the $\mathrm{SN}$ luminosity depends on the amount of ${ }^{56} \mathrm{Ni}$ synthesized in the explosion (e.g., Weaver and Woosley 1980).

Since type IIP SNe are produced by a variety of progenitors, with different masses and chemical compositions, the observed features (luminosity at the plateau, length of the plateau, kinetic energy of the ejecta, amount of synthesized ${ }^{56} \mathrm{Ni}$ ) can be very different from SN to SN (see Fig. 5, from Anderson et al. 2014, here reproduced by kind permission). Indeed, absolute plateau magnitudes range typically from $M_{V}=-15.5 \mathrm{mag}$ to $M_{V}=-18.5 \mathrm{mag}$, initial velocity of the ejecta are of the order of $1-2 \times 10^{4} \mathrm{~km} \mathrm{~s}^{-1}$, and initial photospheric temperatures are of usually around $1-2 \times 10^{4} \mathrm{~K}$.

Nevertheless, interestingly and physically motivated relationships between observable quantities, such as between the linear radius (estimated from the velocity curve) and the 
angular radius (estimated by fitting a black body to the observed fluxes at different epochs); or between the luminosity at the middle plateau and expansion velocity, allow us to use them as "standardized" distance indicators. The first method is known as the expanding photosphere method (EPM, Kirshner and Kwan 1974), while the second has been dubbed the "standardized candle method" (SCM, Hamuy and Pinto 2002). Quite interestingly, the two methods can give consistent results, up to cosmological distances (e.g. Gall et al. 2016).

\subsection{Physical Basis of the EPM and SCM Method}

EPM is actually a variant of the Baade-Wesselink method, able to produce very accurate results (see Bose and Kumar 2014). The method requires the measurement of the temperature of the expanding stellar envelope, and of the envelope radius, which in turn comes from measurement of time since explosion and the expansion speed from the Doppler shift of the lines. Thus, it allows to measure the absolute luminosity, and finally to obtain the luminosity distance in a direct way. Type II SNe are intrinsicaly bright, so this method is able to provide distance estimates up to cosmological distances, independently from the adopted distance ladder, thus providing an independent check of the results obtained, for example, with the type Ia SNe, and it can be applied at any phase. However, it is observationally demanding, since it requires multi-band photometric data and good quality spectra. Moreover, some modeling is needed, especially in correctly estimating the dilution factor of $\mathrm{SNe}$ atmospheric models respect to a pure black body. A further improvement of the EPM is the spectral fitting expanding atmosphere method (SEAM, Mitchell et al. 2002), based on full NLTE atmospheric code. It can give quite accurate and precise results (e.g. Baron et al. 2004; Bose and Kumar 2014), but it is computationally intensive and requires high S/N spectra at early phases.

SCM was introduced by Hamuy and Pinto in 2002 (Hamuy and Pinto 2002, hereafter HP02) as an empirical correlation between the SN luminosity and the expansion velocity of the ejecta. They calibrated their luminosity-velocity relation at day 50 in the $V$ and $I$ photometric bands, which corresponds to a middle-plateau phase for most of the type IIP SNe. Subsequently, Kasen and Woosley (2009) provided theoretical basis on the SCM. As a matter of fact, SCM is a simple recasting of the Baade-Wesselink method. Indeed, since the expansion is homologous (and therefore the velocity is proportional to the radius), the luminosity $L$ can be written as $L=4 \pi v_{p h}^{2} t^{2} \zeta^{2} T_{p h}^{4}$, where $v_{p h}$ and $T_{p h}$ are the photospheric velocity and temperature, respectively; $\zeta$ is a dilution factor, which accounts for the departure from a perfect blackbody, while $t$ is the reference epoch. Now, $t$ can be arbitrarily chosen (50 day by construction), and $T_{p h}$ is a good proxy of the temperature $T_{H}$ of the hydrogen recombination, $T_{h} \approx 6000 \mathrm{~K}$, nearly a constant along the plateau. Finally, the dilution factor $\zeta$ can be estimated from NLTE models, but it is a strong function of the luminosity, and it can be absorbed in the exponent. It should be explicitly noted that SCM does not need to be applied necessarily at day 50, and that similar relations are valid all along the plateau. However, at epochs earlier than day 30 the ejecta temperature is too high, and probably the approximation $T_{p h} \sim T_{h}$ is not valid. Moreover, the atmospheric velocity curve is rapidly changing during the first 40-50 days, and a 10-15 days uncertainty in the explosion epoch can reflect in a substantial bias in the velocity curve.

\subsection{Current Calibrations: an Overview}

The first calibration was given by HP02 that, on the basis of 17 literature type IIP SNe, 8 of which well embedded in the Hubble flow, derived a relation in the $V$ and the $I$ band 
as a function of the photospheric velocity and of the redshift. Their calibration showed a scatter of the order of $9 \%$, comparable with the precision of 7\%, typical of type Ia SNe. However, an estimate of the absorption is needed, and this is usually a thorny problem when dealing with SNe. The problem was faced by Hamuy (2003), where the absorption was estimated on the basis of observed $(V-I)$ colors. Indeed, when it is assumed that the intrinsic end-of-the-plateau $(V-I)$ color is the same for all the type IIP SNe and it is a function of the photospheric temperature only, a possible $(V-I)$ color excess is due to the host galaxy extinction. The underlying physical assumption is that in type IIP SNe the opacity is mainly caused by the $\mathrm{e}^{-}$scattering, so that they reach the same hydrogen recombination temperature as they evolve. However, some discrepancies are obtained, probably due to metallicity variations from one SN to the other. Moreover, as pointed out by Nugent et al. (2006), a color-based extinction correction is impractical for faint (i.e. basically distant) $\mathrm{SNe}$, since it would require a continuous monitoring to catch the end of the plateau, before the luminosity drop.

A subsequent calibration was then proposed by Nugent et al. (2006) (N06), where an extinction correction was determined from the rest-frame $(V-I)$ color at day 50, adopting a color-stretch relationship, as done in Ia SNe studies. A standard $R_{V}=3.1$ dust law was used. Moreover, since at moderate redshifts the weak Fe II $\lambda 5169$ could be hardly measured (because they can be redshifted into the $\mathrm{OH}$ forest), they explored the practicality of stronger lines, such as the $\mathrm{H} \beta$. Also, they derived an useful empirical relation to scale the observed Fe II $\lambda 5169$ velocity at a given epoch, to the reference +50 day. They obtained the first Hubble diagram at cosmologically relevant redshifts $(z \sim 0.3)$ with a rms scatter in distance of $13 \%$, which is comparable with the rms scatter obtained with Ia SNe.

Poznanski and coworkers (Poznanski et al. 2009, hereafter P09) used a fitting method similar to those adopted by N06, but taking the extinction law as a free paramenter. This procedure yielded a mild total-to-selective absorption ratio $R_{V}=1.5$ and, after discarding a few outliers with faster decline rates, they finally obtained a scatter of $10 \%$ in distance.

Subsequently, Olivares et al. (2010) adopted as a reference epoch a "custom" -30 day from the half of the luminosity drop, to take into account the different length of the plateau phase from SN to SN. By allowing $R_{V}$ to vary (and confirming a low $R_{V}=1.4 \pm 0.1$ ), they found again that SCM can deliver Hubble diagrams with rms down to 6\%-9\%. Interestingly, after calibrating their Hubble diagrams with the Cepheid distances to SN 1999em (Leonard et al. 2003) and SN 2004dj (Freedman et al. 2001b), they obtained a Hubble constant in the range $62-105 \mathrm{~km} \mathrm{~s}^{-1} \mathrm{Mpc}^{-1}$, but with an average value of $69 \pm 16 \mathrm{~km} \mathrm{~s}^{-1} \mathrm{Mpc}^{-1}$ in the $V$-band, and similar values in the $B$ and $I$-bands. The large scatter reflects the fact that only two calibrating $\mathrm{SNe}$ were employed, but the average value is very close to our most precise estimate of the Hubble constant, $H_{0}=73.24 \pm 1.74 \mathrm{~km} \mathrm{~s}^{-1} \mathrm{Mpc}^{-1}$ (Riess et al. 2016).

D'Andrea et al. (2010) adopted $K$-corrections to determine rest-frame magnitudes at day 50 for 15 SDSS II SNe, spanning a redshift range between $z=0.015$ and $z=0.12$. They also took into account the rest-frame epoch $50(1+z)$. Their best-fit parameters differed significantly from those obtained by P09, and they attributed the discrepancy to the fact that their SNe sample could be intrinsically brighter than those of P09. Moreover, they concluded that a major source of systematic uncertainty in their analysis was probably due to the difficulty of accurately measuring the velocity of the Fe II $\lambda 5169$ feature, and to the extrapolation of the velocity measured at early epochs to later phases. Finally, they warned that the template database should be extended, in order to perform a reliable $K$-correction.

Maguire et al. (2010) extended the SCM to the near-infrared bands, since at those wavelengths both the extinction and the number of spectral lines are lower. The latter aspect implies that NIR magnitudes are less affected by differences in strength and width of the 
lines (i.e. less sensitive to metallicity effects), from SN to SN. Even though their adopted sample contained only $12 \mathrm{SNe}$, they demonstrated that using JHK magnitudes it is possible to reduce the scatter in the Hubble diagram down to $0.1-0.15 \mathrm{mag}$, with the error in the expansion velocity being the major source of uncertainty.

\subsection{Discussion and Final Remarks}

The extragalactic distance scale up to cosmological distances is intimately connected with type Ia SNe, and through type Ia SNe the acceleration of the Universe was discovered (Perlmutter et al. 1999b; Riess et al. 1998b; Schmidt et al. 1998b). At the present time, current facilities allow us to detect and study type Ia SNe up to $z \sim 1.9$ (Rubin et al. 2013; Jones et al. 2013), and recently up to 2.3 (Riess et al. 2017), while the next generation of extremely large telescopes will allow us to study type Ia SNe up to $z \sim 4$ (Hook 2013). At high $z$, however, the number of type Ia SNe may significantly decrease, due to the long lifetimes of their progenitors. Alternatively, the ubiquitous type II (core-collapse) SNe could be an appealing choice to probe further cosmological distances. Moreover, since type II SNe are produced essentially by young stellar populations, they may constitute a more homogeneous sample, than type Ia SNe, with respect to the age of the stellar population. However, it should be noted that they are sgnificantly fainter, and that their study could be more difficult, since they may explode in younger and dustier regions, and this especially holds at cosmological distances, in a general younger environment. On the other side, they are expected to be more abundant per unit volume (Cappellaro et al. 2005; Hopkins and Beacom 2006).

All the current calibrations of SCM basically rely on a sample of type IIP SNe spanned in a range in $z$, for which magnitudes and expansion velocities were available. The major uncertainties are:

- explosion epoch: unless a very early detection and very good sampling of the phenomenon is available, since the method requires a common reference epoch (day +50 , but other choices are allowed, see Olivares et al. 2010), an uncertainty in the explosion epoch reflects in a scatter in the derived calibration, especially because the expansion velocity rapidly changes during the first $\approx 30$ days;

- reddening correction: only for bright $\mathrm{SNe}$ explosions in nearby galaxies sound estimates of the local reddening are available, for example via the NaI D. In all the other cases, we must rely on color corrections whose precision is of the order of 0.4 mag in $(V-I)$ (Olivares et al. 2010). However, this problem has a much lower impact in the NIR bands;

- velocity of the ejecta: the Fe II $\lambda 5169$ can be difficult to be measured, especially for faint and/or distant $\mathrm{SNe}$, and for the bright $\mathrm{SNe}$ the typical uncertainties are of the order of $150 \mathrm{~km} \mathrm{~s}^{-1}$, that is uncertainties of the order of $2-3 \%$ in the measure (at day 50). Given the typical slopes of the SCM, of order of 5-6 in the $I$-band, the final contribution to the error budget is of the order of 10-20\%;

- $K$-corrections: when calibrating the SCM in a given photometric band using $\mathrm{SNe}$ at different $z$, it should be noted that the photons collected in the observed band, for a given distant $\mathrm{SN}$, actually come from a different wavelength. This means that we are using photons coming from intrinsically different forms of the SCM, with different slopes. Therefore, if $K$-corrections are not applied, the observed relationships are likely to be affected by a larger scatter. Moreover, since the published calibrations are based on SNe spanning different ranges in $z$, this could also affect the coefficients. However, D'Andrea et al. (2010) correctly adopted $K$-corrections. 
Fig. 6 Original HP02 plane, with the positions of the homogeneously studied $\mathrm{SNe}$ 2012A (blue), 2012aw (red) and 2012ec (black). Plot from Barbarino et al. (2015), reproduced by kind permission

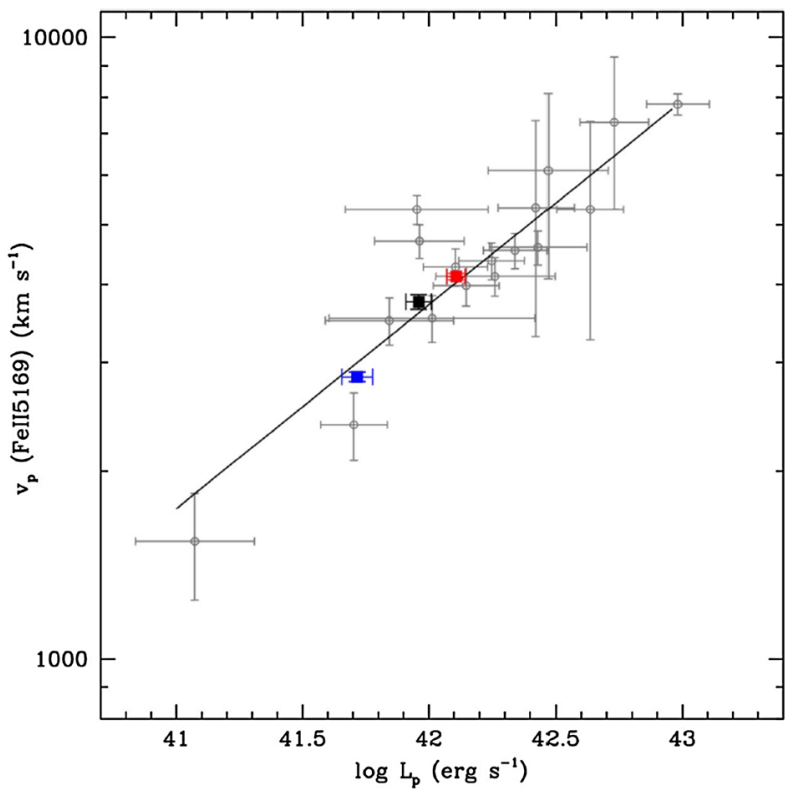

It should be noted that, when nearby $\mathrm{SNe}$ are used, with a tight sampling of their evolution and a homogeneous technique of analysis is employed, the observed scatter of the SCM would be greatly reduced, as suggested in Barbarino et al. (2015). In Fig. 6 we reproduce their Fig. 20, where the position of the SNe 2012A, 2012aw and 2012ec is shown in the original Hamuy and Pinto plane.

At the present time, we still lack a homogeneous and sound calibration of the SCM based only on the primary distance indicators and, on the other side, we have only a few numbers of host galaxies where type IIP SNe have been exploded and also we detected Cepheids and/or the TRGB. A first step toward this direction was provided by Hamuy (2004) and by Jang and Lee (2014), where they adopted the calibrations of the SCM published by HP02 and Olivares et al. (2010), respectively, with the distances provided by Cepheids and TRGB, to estimate the Hubble constant. More recently, Polshaw et al. (2015) applied the SCM to SN 2014bc, exploded in the anchor galaxy NGC 4258, for which a geometric maser distance (with an uncertainty of only 3\%, Humphreys et al. 2013) and a Cepheids distance (Fiorentino et al. 2013) is available. They applied almost all the currently available calibrations of the SCM to SN 2014bc, and compared the estimated distances with both the maser and the Cepheids distances. They found some discrepancies between the SCM-based distance moduli and the maser distance modulus, ranging from -0.38 mag to $0.31 \mathrm{mag}$. To further investigate the scatter among the available calibrations, they applied the SCM to a set of 6 type IIP SNe occurred in galaxies for which Cepheid distances were available. They obtained a Hubble diagram in the $I$-band with a small scatter $\left(\sigma_{I} \sim 0.16 \mathrm{mag}\right)$, and the following SCM calibration:

$$
\begin{aligned}
I_{50} & -A_{I}+5.665( \pm 0.487) \log \left(v_{50} / 5000\right) \\
& =5 \log \left(H_{0} D\right)-2.045( \pm 0.137)
\end{aligned}
$$

where $H_{0}=73.8 \mathrm{~km} \mathrm{~s}^{-1} \mathrm{Mpc}^{-1}$ (Riess et al. 2011), and $D$ is the distance. 
This calibration relies on the cosmic distance ladder, even if based on only a few objects. However, the derived SCM is based on Cepheid distances based on different calibrations of the Cepheid period-luminosity relations. The differences among the various calibrations are typically of the order of $0.1 \mathrm{mag}$ (e.g. Fiorentino et al. 2013, and references therein). Moreover, also the adopted reddenings came from different sources, even from different calibrations of the same $\mathrm{NaI} D$ feature. To this aspect, we point out that current calibrations of the NaI $\mathrm{D}$ feature in the same galaxy may provide differences up to $\Delta E(B-V) \sim 0.15$ mag (see the discussion in Dall'Ora et al. 2014).

A further development of a calibration of the SCM based on primary distance indicators (Cepheids, TRGB) is highly desirable, with a larger number of calibrators and with a homogeneous analysis (i.e. same estimate of the reddening and same Cepheids period-luminosity relations and TRGB calibrations). Moreover, since the SNe calibrated on primary distance indicators occur in the local Universe, they are likely to be deeply investigated. Moreover, the progenitor could be detectable on archive images. This would allow us to fully explore the space of the structural parameters that could affect the SCM. Indeed, very recently SN LSQ13fn (Polshaw et al. 2016) was found to break the standardized candle relation. A possible explanation for that could be the low metallicity of the progenitor $\left(\sim 0.1 Z_{\odot}\right.$, Polshaw et al. 2016). As a matter of fact, theoretical models (Kasen and Woosley 2009) predict a metallicity dependence, but at the $0.1 \mathrm{mag}$ level, not as large as observed in the case of SN LSQ13fn (almost $2 \mathrm{mag}$ ). However, a possible explanation could be a combined effect of low-metallicity of the ejecta and a strong circumstellar interactions. Whatever the case, detailed studies of nearby type IIP SNe, spanning a range of masses, metallicities and environments are of extreme importance to fully characterize the SCM.

As a final point we note that future facilities, such as E-ELT and NGST, will allow us to extend the range of the cosmological type IIP SNe, on which SCM could be applied, but also the range of local SNe, to calibrate the SCM. However, these forthcoming facilities will operate at the NIR wavelengths, therefore making it essential a sound NIR calibration of the SCM.

\section{Tully-Fisher and Faber-Jackson Methods}

Tully-Fisher method is a historically important method based on the empirical relation between the intrinsic luminosity of a spiral galaxy and the emission line width (Tully and Fisher 1977). This method opened a way to measure distances and proper motions for spiral galaxies and clusters of galaxies. The method was calibrated using Cepheid stars in nearby spiral galaxies, and then could be easily extended up to $\sim 100 \mathrm{Mpc}$. For over 20 years this was the most popular method for probing this distance range, but with invent of new methods, particularly SN Ia, it's role in cosmology strongly diminished due to considerable intrinsic scatter. This scatter can be only partially reduced when replacing the optical luminosity with IR emission, molecular hydrogen or CO. Thus, in recent years the Tully-Fisher relation was rather used to probe the mass to luminosity (M/L) variation in galaxies with known distances, rather than as a distance estimator in itself (e.g. Davis et al. 2016). In principle, once a reference sample for each morphological type is determined then one could use it to estimate distances in the usual way, but at this stage it is not yet expected to give reliable results.

Like a Tully-Fisher, Faber-Jackson relation (Faber and Jackson 1976) is an empirical relation between the intrinsic luminosity and the stellar velocity dispersion in the elliptical galaxies. However, this method has vary large intrinsic scatter although it was based on some 
pre-selection of morphological types, and the actual relation between the galaxy kinematics and morphology is rather complex (see e.g. Cortese et al. 2016). Nevertheless, more general version of this relation known as Fundamental plane (Dressler et al. 1987) can be applied to determine the distances in large sample of galaxies (e.g. da Cunha et al. 2017).

\section{Surface Brightness Fluctuations}

In 1988, Tonry and Schneider developed a new technique for measuring extragalactic distances based on the spatial luminosity variations in early-type galaxies (Tonry and Schneider 1988). The method, known as Surface Brightness Fluctuations (SBF) works because the variation in brightness from pixel-to-pixel varies as the square root of the number of stars per pixel, and thus galaxies at larger distances will have smaller variations, appearing smoother, than nearby galaxies (see Blakeslee 2013, for a recent review of the SBF technique and distance measurements).

Making accurate SBF measurements requires a solid anchor to the distance ladder for calibration. In principle, one could determine the SBF distance calibration based on theoretical modeling of stellar populations; in practice, it is more common to adopt an empirical calibration, using Cepheid distances to set the zero point, and the observed variation of SBF magnitude with color to calibrate stellar population effects. In addition to measuring distances, SBF has also been used to explore the properties of unresolved stellar populations in galaxies with known distances, which is valuable in constraining stellar population models (see Jensen et al. 2015, for details).

The ground-based SBF technique was initially calibrated for use at optical wavelengths ( $I$ and $z$ ) and in the near-IR ( $J, H$, and $K$-bands). At $I$, the effects of age and metallicity on SBF brightness are largely degenerate, making it possible to calibrate distances for a wide variety of early-type galaxies using a single broadband color. The near-IR bands exhibit more scatter in SBF as a function of galaxy color, but the fluctuations themselves are much brighter because the stellar light in old populations is dominated by red giant branch stars, which are brighter in the near-IR. Even though the IR background is much higher, the brighter fluctuations and better seeing (from the ground) typically make it possible for IR $\mathrm{SBF}$ to reach much greater distances.

The first ground-based SBF surveys were largely limited to distances of about $20 \mathrm{Mpc}$ (e.g., Tonry et al. 2001). During the last decade, new instruments on the Hubble Space Telescope $(H S T)$ have enabled us to achieve unprecedented precision with the SBF method and push to much larger distances, thanks to their high spatial resolution, point spread function (PSF) stability, low background levels, and relatively wide fields of view. The Advanced Camera for Surveys (ACS) was used to conduct extensive surveys of the Virgo and Fornax clusters, from which a calibration of the $z$-band SBF distance method with statistical scatter of 0.08 mag was achieved, corresponding to $4 \%$ in distance (Mei et al. 2007; Blakeslee et al. 2009). This puts SBF on par with the most accurate extragalactic distance indicators, including Type Ia supernovae ( $\mathrm{SNe}$ ) and Cepheids.

Jensen et al. (2015) have recently established a new SBF calibration for the F110W and F160W filters ( $J$ and $H$ bands) of the WFC3/IR camera, which can routinely measure SBF distances to $80 \mathrm{Mpc}$ in a single $H S T$ orbit. The new IR SBF calibration is based on the ACS SBF distances to Virgo and Fornax galaxies, and is tied to the Cepheid distance scale. They find a statistical scatter of $0.1 \mathrm{mag}$ ( $5 \%$ in distance) per galaxy for redder ellipticals, with greater variation in bluer and lower-luminosity galaxies. Comparison with stellar population models implies that redder ellipticals contain old, metal-rich populations, as expected, 
and that bluer dwarf ellipticals contain a wider range of stellar population ages and lower metallicities, with the youngest populations near their centers. IR color gradients appear to be closely related to age, so IR SBF distance measurements are best limited to the reddest and oldest high mass elliptical galaxies.

A team of astronomers is now using WFC3/IR (PI J. Blakeslee) to measure IR SBF distances to a sample of 34 high-mass early-type galaxies in the MASSIVE survey (Ma et al. 2014). The goal of the MASSIVE survey is to better understand the structure and dynamics of the 100 most massive galaxies within $\sim 100 \mathrm{Mpc}$ using a wide array of imaging and spectroscopic techniques. Of particular interest is measuring the masses of the central supermassive black holes in these galaxies, for which accurate distances are necessary. The IR SBF distances will also remove peculiar velocity errors and better constrain cosmic flows within $100 \mathrm{Mpc}$.

The power of SBF as a tool for cosmology is now being established with a new HST program to measure IR SBF distances to a collection of early-type supernova host galaxies (PI P. Milne). The goal of this project is to reduce systematic uncertainties in Type Ia SNe luminosities and explore possible environmental effects on the brightnesses of Ia SNe that are typically calibrated locally using Cepheids in late-type spirals, but are more often observed in early-type galaxies at high redshift. IR SBF is the only method that reaches large enough distances to observe the host galaxies and measure their distances with the requisite precision.

Accomplishing the goals of these projects relies on efficient, high-accuracy distance measurement that is currently only possible with $H S T$ resolution, and can be accomplished most efficiently with WFC3/IR. The future of IR SBF is not limited to HST, however. New AO systems on large telescopes such as the multi-conjugate AO system "GeMS" on the GeminiSouth telescope provide a highly stable and uniform PSF over a wide $\left(\sim 2 \operatorname{arcmin}^{2}\right)$ field of view. Initial GeMS observations of three galaxies have demonstrated that the SBF signal can be measured with high fidelity in modest exposure times out to $100 \mathrm{Mpc}$ using $\sim 0.08$ arcsec FWHM $K$-band images.

There are plans to continue to develop the AO SBF techniques with the expectation that the next generation of large telescope (e.g., TMT, GMT, and E-ELT) with wide-field AO systems will make reliable IR SBF distance measurements out to several hundred Mpc. The James Webb Space Telescope also has great potential to push the IR SBF technique to distances of perhaps $500 \mathrm{Mpc}$.

\section{Active Galactic Nuclei}

Active Galactic Nuclei (AGN) are not the objects frequently discussed in the context of distance measurements. However, an excellent example of the water maser shows their current importance, and the continuously increasing AGN samples and our understanding of those objects open new possibilities.

\subsection{Water Masers}

The discovery of a water maser at $22.23508 \mathrm{GHz}$ in the Seyfert II galaxy NGC 4258 (Miyoshi et al. 1995; Herrnstein et al. 2005) offers an unprecedented possibility to measure the distance to this galaxy directly through geometrical methods, without any need for intermediate steps, and with high accuracy. 
This coherent emission forms due to collisional excitation (for emission mechanism, see e.g. Elitzur 1992; Lo 2005), the emitted frequency corresponds to the transition between rotational energy levels in the water molecule. The emission may form in the medium with the temperature about $300-600 \mathrm{~K}$, and particle number densities $10^{8}-10^{11} \mathrm{~cm}^{-3}$. Its narrow band emission traces precisely the dynamics of the material through the line shifts.

In general, water maser emission can form in molecular clouds, comets, planetary atmospheres, stellar atmospheres, and in the case of distant galaxies can be mostly found in starburst regions. The maser emission in NGC 4258 is different, it originates in a Keplerian slightly warped disk surrounding the central black hole and it is strong enough that it can be mapped at sub-milli-arc-second resolution by Very Long Baseline Interferometry (VLBI), providing a powerful tool to probe spatial and kinematic distribution of the molecular gas at distances below $1 \mathrm{pc}$ from the central black hole. The resolved image of the emission allows for the measurement of the spacial distribution of the emission, and the wavelength shifts give the velocity, and it was shown already by Miyoshi et al. (1995) that the measured radial profile corresponds to the Keplerian motion. Further measurement of the proper motion, or acceleration, thus opens a way to the measurement of the geometrical distance. Subsequent long-term monitoring of this source (Argon et al. 2007; Humphreys et al. 2013) allowed to obtain the distance to NGC 4258 with the accurracy of $3 \%$. New analysis of the same data by Riess et al. (2016) gave the distance measurement to this source:

$$
D(\text { NGC } 4258)=7.54 \pm 0.17(\text { random }) \pm 0.10(\text { systematic }) \mathrm{Mpc},
$$

or, equivalently, magnitude distance of $29.39 \pm 0.06$ mag. Here the statistical error is the value coming directly from the Monte Carlo Markov Chain code, used for fitting the parameters, and the statistical error comes from the same code run for various initial values. This result, combined with a large number of Cepheid stars discovered in NGC 4258 (Fausnaugh et al. 2015) and in several hosts of SN Ia, opened a way to determine the local value of the Hubble constant $H_{0}=72.25 \pm 2.38 \mathrm{~km} \mathrm{~s}^{-1} \mathrm{Mpc}^{-1}$ with $2.4 \%$ accuracy (Riess et al. 2016).

The water maser in NGC 4258 is not the only one detected in an active galaxy, and not even the first one. The first water maser was detected in 1979 in NGC 4945 (Dos Santos and Lepine 1979), and a number of water masers are known till now, although none of those sources have such unprecedented data quality from the point of view of a distance measurement. However, statistical use of numerous sources (Megamaser Cosmology Project; Braatz et al. 2015) is expected to provide a measurement precision of $\sim 5 \%$ on the Hubble constant.

\subsection{Continuum Reverberation}

It is widely thought that accretion onto the supermassive black holes in bright AGN takes place via an optically thick, geometry thin accretion disk described by Shakura and Sunyaev (1973). Each annulus within such a disk will emit like a blackbody with temperature $T$ at radius $R$. An accretion disk dominated by heating from viscous dissipation which is around a black hole of mass $M$ accreting at a rate $\dot{M}$, has a temperature profile given by

$$
T(R)=\left(\frac{3 G M \dot{M}}{8 \pi R^{3} \sigma}\right)^{1 / 4},
$$

when the radius is much greater than the innermost stable circular orbit. The disk's absolute flux is then given by summing up blackbodies, $B_{v}$, over all disk annuli: 


$$
\begin{aligned}
f_{v}(\lambda) & =\int B_{v}(T(R), \lambda) \frac{2 \pi R d R \cos i}{D^{2}} \\
& =\left(\frac{1200 G^{2} h}{\pi^{9} c^{2}}\right)^{1 / 3} \frac{\cos i(M \dot{M})^{2 / 3} v^{1 / 3}}{D^{2}} .
\end{aligned}
$$

Therefore, if the temperature profile of the disk can be measured, the observed and expected fluxes can be compared in order to determine the distance. The key question, then, is how to determine the temperature profile of the disk. The accretion disk, expected to be a few light days across, is too small to be spatially resolved, and thus indirect techniques must be used. Reverberation mapping (Blandford and McKee 1982; Peterson 2014a) uses light travel time to measure spatial separations within a distant accretion flow. Much in the same way the continuum variability studies of AGN provide a means to probe the accretion disk around the central supermassive black hole and allow measurement of the temperature profile of the disk (for a detailed description see Collier et al. 1999; Cackett et al. 2007). High energy Xray/EUV photons produced close to the compact object irradiate the surrounding gas which reprocesses this into UV/optical continuum, with the hotter inner regions emitting mainly UV photons and seeing variations in the irradiating flux before the cooler outer regions, which emit mainly optical photons. As the ionizing radiation varies erratically, so do the reprocessed components but with time delays due to light travel time within the systemthe light travel time from source to reprocessing site to observer is longer than that on the direct path from source to observer. These observable delays provide indirect information on the size and structure of the surrounding accretion flows. This technique is a powerful probe of accretion flows in AGN.

Thermal radiation from a disk annulus at temperature $T(R)$ emerges with a range of wavelengths, $\lambda \sim h c / k T(R)$. Roughly speaking, each wavelength picks out a different temperature zone and the time delay $\tau=R / c$ measures the corresponding radius. Thus, in this reprocessing scenario the accretion disk reverberates and we expect to observe correlated variability between all continuum bands, with the shortest wavelength lightcurves varying first.

More specifically, the observed delays between different continuum wavelengths depend on the disk's radial temperature distribution $T(R)$, its accretion rate, and the mass of the central black hole. A disk surface with $T \propto R^{-b}$ will reverberate with a delay spectrum $\tau \propto$ $\lambda^{-1 / b}$. For the temperature distribution of a steady-state externally irradiated disk, $T(R) \propto$ $R^{-3 / 4}$ (as given above), thus the wavelength-dependent continuum time delays should follow

$$
\tau=\frac{R}{c} \propto(M \dot{M})^{1 / 3} T^{-4 / 3} \propto(M \dot{M})^{1 / 3} \lambda^{4 / 3} .
$$

Accretion disk reverberation therefore allows the measurement of the disk temperature profile, and so can be used to determine the AGN distance (Collier et al. 1999) via:

$$
D=3.3\left(\frac{\tau}{\text { days }}\right)\left(\frac{\lambda}{10^{4} \AA}\right)^{-3 / 2}\left(\frac{f_{v} / \cos i}{\mathrm{Jy}}\right)^{-1 / 2} \mathrm{Mpc} .
$$

Here, $f_{v}$ must be the accretion disk flux, which can be obtained by taking difference spectra to isolate the variable component of AGN light.

Over the past 20 years many studies have searched for the expected signatures and found good correlations between different UV and optical wavelength-bands, but the expected interband lags were never of high significance (e.g., Edelson et al. 1996; Wanders et al. 1997; Collier et al. 1998, 2001; Sergeev et al. 2005). Importantly, though, Sergeev et al. 
Fig. 7 Time delay versus rest-wavelength for NGC 5548 (adapted from Fausnaugh et al. 2016). The solid line shows the best-fitting $\lambda^{4 / 3}$ relation, while the dotted line shows the expected lags from a thin disk accreting at $10 \%$ of the Eddington rate

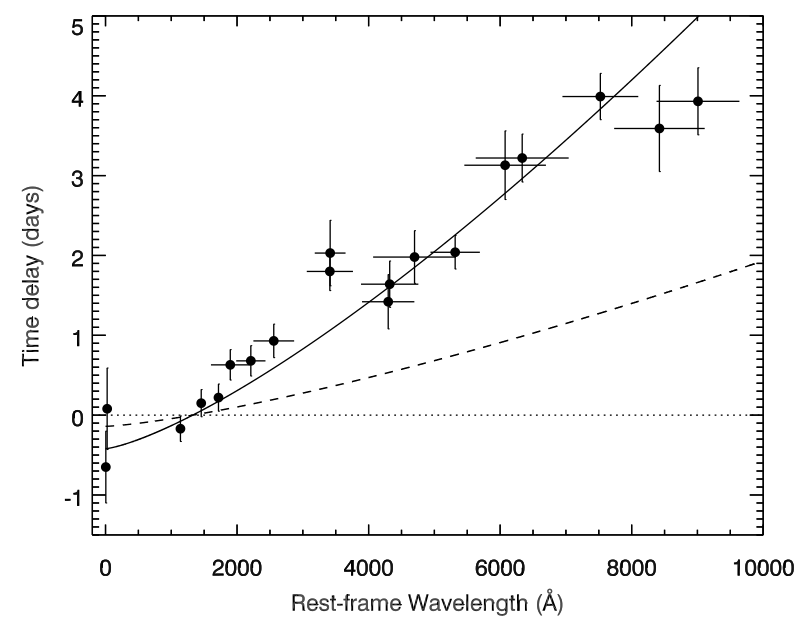

(2005) found that in all the 14 nearby Seyferts in their sample the time-delays are present and show an increase in time delay with increasing wavelength, and the delays increase with greater absolute luminosity of the AGN as predicted by reprocessed disk models. The first attempt to apply this disk reverberation method to determine an AGN distance yielded an $H_{0}$ value a factor of 1.66 smaller than any of the currently considered values (Collier et al. 1999). Cackett et al. (2007) applied this model to fit the lags and fluxes from all 14 AGN in the Sergeev et al. sample, but again found a value of $H_{0}$ a factor of 1.6 too small. One interpretation of this result is that the accretion disks are, on average, a factor of 1.6 larger than predicted by the standard model.

Over the last couple of years, significant progress has been made in better understanding the wavelength-dependent lags. Much improved sampling cadence and long baseline campaigns have allowed for significant lags from X-rays through to the near-IR to be measured in two objects, NGC 2617 and NGC 5548 (Shappee et al. 2014b; McHardy et al. 2014; Edelson et al. 2015; Fausnaugh et al. 2016). Both are consistent with the $\tau \propto \lambda^{4 / 3}$ relation, however, the lags are a factor of $\sim 3$ larger than expected based on the standard disk model (McHardy et al. 2014; Edelson et al. 2015; Fausnaugh et al. 2016). Figure 7 shows the wavelength-dependent lags from NGC 5548.

The challenge now lies in understanding the discrepancy with the disk model. Strong emission lines present in broadband filters can act to lengthen the measured continuum lag, since the broad emission line lag is longer than the continuum lag (Chelouche and Zucker 2013). However, in NGC 5548 this does not appear to be a large effect (Fausnaugh et al. 2016). Furthermore, continuum emission from diffuse gas in the broad line region will also act to lengthen the lag (Korista and Goad 2001), and hints of this effect are present in the NGC 5548 lags, where the u-band (where the Balmer diffuse continuum will peak) lag is an outlier in the otherwise smooth wavelength-dependent lags (Edelson et al. 2015; Fausnaugh et al. 2016). Alternatively, our understanding of disk accretion is incomplete, and other, more complex geometries occur (e.g. Gardner and Done 2016).

Future intense monitoring campaigns will help better understand these differences with the standard disk model. If these discrepancies can be understood, then this method of measuring distances has the potential to be very powerful given the AGN monitoring that will take place in the LSST-era. 


\subsection{BLR Reverberation}

Intense emission lines, most notably Balmer lines, with kinematic width of order of thousands $\mathrm{km} / \mathrm{s}$ are the most characteristic features of AGN. The variability of the emission line intensity has been noticed already by Andrillat and Souffrin (1968), and the response of the lines to the variable intrinsic continuum opened a way to reverberation mapping of the Broad Line Region (e.g., Cherepashchuk and Lyutyi 1973; Gaskell and Sparke 1986; Kaspi et al. 2000; for a review, see Peterson 2014b; Bentz 2015). In the simplest approach, the time delay between the lines and the continuum measures the size of the BLR. Subsequent studies showed that the BLR clouds are predominantly in the Keplerian motion so their orbital velocity, measured through the line width, combined with the orbital radius allows to measure the black hole mass through the virial theorem. This application firmly established the importance of the BLR reverberation for cosmology.

The next important step has been made by Watson et al. (2011) who suggested that BLR reverberation measurement can be also used to determine the distance to the source. AGN are clearly not standard candles, as their intrinsic luminosities span orders of magnitutes, but the reverberation studies showed a tight relation between the delay of the emission line (mosty $\mathrm{H} \beta$ ) and the intrinsic luminosity flux (e.g., Peterson et al. 1999; Kaspi et al. 2000), most frequently measured at $5100 \AA$ since the majority of the reverberation studies were done for low redshift AGN in the optical band. Line delay can be interpreted as a mean/effective radius of the BLR region. Thus this relation, after careful subtraction of the host galaxy reads

$$
\left.\log R_{B L R}=K+\alpha \log L_{44}, \quad \text { [light days }\right]
$$

where $L_{44}$ is the monochromatic $\lambda L_{\lambda}$ flux expressed in units of $10^{44} \mathrm{erg} \mathrm{s}^{-1}$, and the coefficient values from the sample version Clean given in Tab. 14 of Bentz et al. (2013) are $K=1.555 \pm 0.024, \alpha=0.542 \pm 0.027$. The directly measured quantities are the line delay, $\tau$, and the observed flux, $F_{\lambda}$ so the value $\tau / F_{\lambda}^{1 / 2}$ provides the distance indicator (Watson et al. 2011).

The dispersion in the delay-luminosity relation is only 0.13 dex, as measured by Bentz et al. (2013) in Clean variant. Thus, with a large number of objects and broader coverage of the redshift range the method offers a very interesting alternative to SNe Ia.

The unique aspect of the AGN Hubble diagram is that while SN distances are observationally restricted now to less than $z \sim 1.9$ (Riess et al. 2001; Jones et al. 2013), AGN diagram can be extended to much larger redshifts thus covering the full range of distances with a single method. The additional advantage of the use of quasars is that those objects, on average, are not strongly affected by extinction (they clean efficiently their environment), and they do not show significant evolution of the metallicity with redshift so in principle no hidden evolutionary bias should be present in their BLR properties across the redshift space.

The number of AGN studied in the context of reverberation mapping is not yet large, about 60 objects were monitored in $\mathrm{H} \beta$ line (see Du et al. 2015, 2016a, for a recent compilation), and only a handful of sources have other line delays measured. The most distant quasar with tentative time delay, measured for CIV line, is still S5 0836+71 at $z=2.172$ studied through 7-year monitoring by Kaspi et al. (2007). However, many monitoring programs are under way (e.g., Czerny et al. 2013; King et al. 2015; Lira et al. 2016). The use of other lines than $\mathrm{H} \beta$ has the advantage that the measured time delay is then considerably shorter which is important for high $\mathrm{z}$ quasars, where the expected time delay is long due to large black hole mass as well as to the $(1+z)$ scaling of the intrinsic timescale to the observed one. 

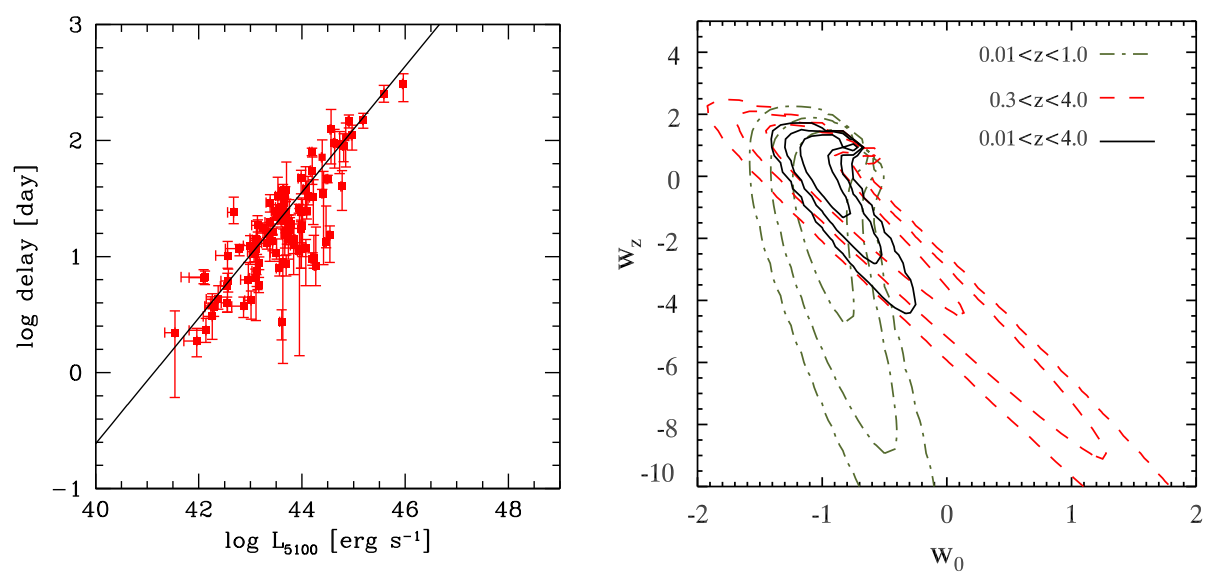

Fig. 8 Left panel: the time delay of $\mathrm{H} \beta$ line as a function of monochromatic luminosity from current reverberation measurements as compiled by Du et al. (2015, 2016a) (red points), and the best fit from Bentz et al. (2013) (see text). Right panel: the expected constraints on dark energy parameters from reverberation-measured 2000 AGN strongly depend on their redshift coverage, and the sources at very low as well as at high redshifts are needed (Fig. 6 from King et al. 2014)

Covering the broad range of redshifts with the same probe is very important for distance determination, and for subsequent cosmological constraints. This is best illustrated by King et al. (2014) where they analyze the expected results from the sample of 2000 AGN. If the dark energy is parametrized as

$$
w(z)=w_{0}+w_{z} z /(1+z)
$$

after Chevallier and Polarski (2001), then to get the strong constraints AGN must populate the broad redshift range $0.01<z<4$, i.e. starting at very low redshifts (see Fig. 8). AGN can cover such a broad range while this is a problem for the methods based on gamma-ray bursts (see Sect. 8).

Nearby AGN are currently monitored by several groups (e.g., Ilić et al. 2015; Barth et al. 2015; Fausnaugh et al. 2016; Bentz et al. 2016a,b; Du et al. 2016a). SDSS quasar sample has been recently monitored for six months within the frame of the SDSS-RM project (Shen et al. 2016) which allowed to provide preliminary estimate of nine $\mathrm{H} \beta$ langs and six $\mathrm{Mg}$ II lags for objects with redshifts above 0.3 , and this program is being extended now, covering 849 sources in total, with cadence of 12 epochs per year. High redshift quasars do not need such a dense sampling but then the project requires at least 5 years of monitoring (Czerny et al. 2013) to measure lags in higher $\mathrm{z}$ sources, and such project is under way within the Oz-DES program (King et al. 2015). Selected sources (771 in the final sample) will be monitored for six years, with 25 measurements for each quasar, on average. Individual delay measurements are expected for half of them, after carefull subtraction of the Fe II and Fe III emission, host galaxy contamination and correction for the reddening. For fainter sources stack analysis will be performed in a number of luminosity bins. Monitoring of a few $z \sim 1$ quasars with as large telescope as SALT will allow for better disentangling of the Mg II line from the underlying Fe II pseudo-continuum (Hryniewicz et al. 2014; Modzelewska et al. 2014).

Narrow-band and broad band reverberation measurements offer an interesting option since they can be done with smaller telescopes and in a more automatic way. In particular, the future Large Synoptic Sky Survey (LSST) will bring 10-year dense coverage of 
thousands of quasars in six photometric bands. The challenge is in disentangling the line and continuum variability but preliminary studies indicate this is possible (e.g., Chelouche et al. 2014).

However, before AGN monitoring can reach maturity in cosmological applications, several issues have to be addressed. First, the AGN Hubble diagram, like SNe Ia, currently requires calibration to obtain absolute distances. This is basically done by adopting the current value of the Hubble constant for nearby AGN. Direct comparison of AGN distances with another distance indicator is rare. One such example is through the detection of 11 Cepheid stars in a Seyfert 1 galaxy NGC 4395 (Thim et al. 2004). However, in principle the calibration issue can be avoided if the understanding of the BLR formation progresses. For example, the idea that the BLR forms as a dust-driven failed wind (Czerny and Hryniewicz 2011) combined with the theory of accretion disk sets the BLR onset at some specific value of $T_{\text {eff }}$ provided by the dust sublimation temperature (see Sect. 6.2). Thus if the hottest dust temperature can be independently measured for a number of AGN this could allow the reverberation method to move to the class of direct methods. Recent comparison of the standard calibration with dust temperature based calibration for a whole AGN sample implies dust temperature $900 \mathrm{~K}$, lower than the hottest dust temperature generally measured in AGN although much higher temperature value was found using the same model from NGC 5548 (Galianni and Horne 2013).

Second aspect is the objective removal of outliers. Some systematic departure of very high Eddington ratio sources from the overall trend has been recently noted ( $\mathrm{Du}$ et al. 2016b). If this conclusion is supported in further studies, such super-Eddington sources have to be removed from the general sample or corrected for the departure trend.

The third problem is related to possible systematic errors when we move towards more distant, brightest quasars. The extension of the power law dependence between $R_{B L R}$ and the monochromatic flux in a form of a simple power law has not been observationally tested. If the BLR formation is well explained by the failed dust model, expected departures from the linear trend are not strong but if the BLR radius mostly depend on the total ionizing flux then the decrease in ionizing photons with the black hole mass (larger at larger distances) may cause strong nonlinear behavior. The measured monochromatic flux is also affected by the viewing angle, usually unknown. This is not a large problem for nearby AGN since the viewing angles of type $1 \mathrm{AGN}$ are limited to the range between 0 and $\sim 45$ deg due to the presence of the dusty torus, and the lag independence on the viewing angle was demonstrated by Starkey et al. (2016). However, if the torus opening decreases with redshift and/or with luminosity it may lead to some systematic errors. Recent studies imply some dependence of dust coverage on luminosity (e.g., Ichikawa et al. 2017) but the opposite conclusion has been reached by Mateos et al. (2017), so more future studies along this line are still needed.

Therefore, the BLR reverberation method has not yet reached maturity. On the other hand, it has a large future potential. Large samples of reverberation-studied sources are coming. Extension of the method to photometric reverberation (Haas et al. 2011) opens a way for future use of the Large Synoptic Sky Survey (LSST) data for this purpose, which will bring 10 years of quasar monitoring, with the cadence of 100 observations per year in 6 photometric channels. In the meantime, systematic problems can be studied with increasing sample of individual, well spectroscopically monitored objects.

\subsection{Extragalactic Distances Based on Dust Reverberation of AGNs}

The inner radius of the dust torus in an active galactic nuclei (AGN) is considered to be determined by sublimation of dust and is proportional to the square-root of the accretion- 
disk luminosity. If its physical size can be measured by reverberation of dust emission, then the luminosity distance of an AGN can be obtained. The Hubble constant was estimated from the distances based on the dust reverberation for local AGNs as $73 \mathrm{~km} \mathrm{~s}^{-1} \mathrm{Mpc}^{-1}$, which shows good agreement with its current standard estimates.

Near-infrared interferometry recently begins to be able to measure the angular scale of the innermost dust torus for brightest AGNs, and the angular diameter distance can be obtained by comparing the reverberation radius with it.

\subsubsection{Luminosity Distance Based on the Dust Reverberation}

Many observations have indicated that an obscuring dust torus surrounds an accretion disk and broad emission-line region (BLR) in the center of an active galactic nucleus (Antonucci 1993). Since dust is sublimated in the vicinity of the accretion disk by absorbing its strong UV-optical continuum emission, the inner radius of the dust torus is considered to be determined by sublimation of dust. The dust sublimation radius $r_{\mathrm{d}}$ can be derived from the radiation-equilibrium equation for the dust grain,

$$
\pi a^{2} \int Q_{v} \frac{L_{\nu}^{\mathrm{AD}}}{4 \pi r_{\mathrm{d}}^{2}} d v=4 \pi a^{2} \int Q_{\nu} \pi B_{v}\left(T_{\mathrm{sub}}\right) d v
$$

where $T_{\text {sub }}$ is the dust sublimation temperature, $a$ is the dust grain size, $Q_{\nu}$ is the absorption efficiency of dust, and $B_{v}$ is the Planck function (Barvainis 1987). The parameters $T_{\text {sub }}$ and $Q_{v}$ at the innermost region of the dust torus would be common in AGNs, because only the dust grains with highest sublimation temperature can survive there and those parameters are determined by the properties of such dust grains. Therefore, the inner radius of the dust torus is expected to be proportional to the square-root of the accretion-disk luminosity. Inversely, the absolute luminosity can be estimated once the inner radius of the dust torus is obtained observationally, which could be used as a distance indicator for AGNs.

The dust reverberation enables us to obtain the inner radius of the dust torus by measuring the lag between the flux variation of the UV-optical continuum emission from the accretion disk and that of the near-infrared thermal emission from the dust torus. A possible application of the dust reverberation of AGNs to the cosmological distance measurement was proposed at the end of the 20th century (Kobayashi et al. 1998; Oknyanskij 1999; Yoshii 2002), and the group of the University of Tokyo started the Multicolor Active Galactic NUclei Monitoring (MAGNUM) project (PI: Yuzuru Yoshii) in 1995 to establish the distance indicator based on the dust reverberation of AGNs and to constrain the cosmological parameters (Yoshii 2002).

Figure 9 shows the radius-luminosity relation for the dust torus obtained by the largest systematic dust reverberation survey for 17 local Seyfert galaxies performed by the MAGNUM project (Koshida et al. 2014). The inner radius of the dust torus clearly correlates with the optical luminosity of AGNs as theoretically expected, which demonstrates the feasibility of the luminosity distance indicator based on the dust reverberation. Then, the dust sublimation model at the innermost dust torus was built to obtain the distances for the 17 AGNs without any distance ladder, and the distances were compared with the recession velocities to estimate the Hubble constant (Yoshii et al. 2014). Figure 10 shows the Hubble diagram. The Hubble constant was estimated approximately as $H_{0}=73 \mathrm{~km} \mathrm{~s}^{-1} \mathrm{Mpc}^{-1}$, which shows good agreement with its current standard estimates. In addition, the distance calibration based on the dust sublimation model in Yoshii et al. (2014) was consistent with that obtained from the distances of the SNe Ia occurred in the AGN host galaxies (Koshida et al. 2017). These results indicate that the distance indicator based on the dust reverberation is a promising new tool for investigating the expanding Universe. 
Fig. 9 Radii of the innermost dust torus and the BLR plotted against the $V$-band luminosity (Koshida et al. 2014). The red filled circles represent the reverberation radii for the dust torus obtained by the MAGNUM project, and the red solid line represents the best-fit regression line for them. The blue crosses represent the reverberation radii for the BLR and the blue dot-dashed line represents the best-fit regression line for them (Bentz et al. 2009). The purple open squares represent the interferometric radii in near-infrared for the dust torus (Kishimoto et al. 2011; Weigelt et al. 2012)

Fig. 10 Hubble diagram for galaxies with the dust reverberation distance (red filled circles) and the Cepheid-variable distance (green filled circles) from Yoshii et al. (2014). The solid line represents the best-fit regression line for the data based on the dust reverberation distance
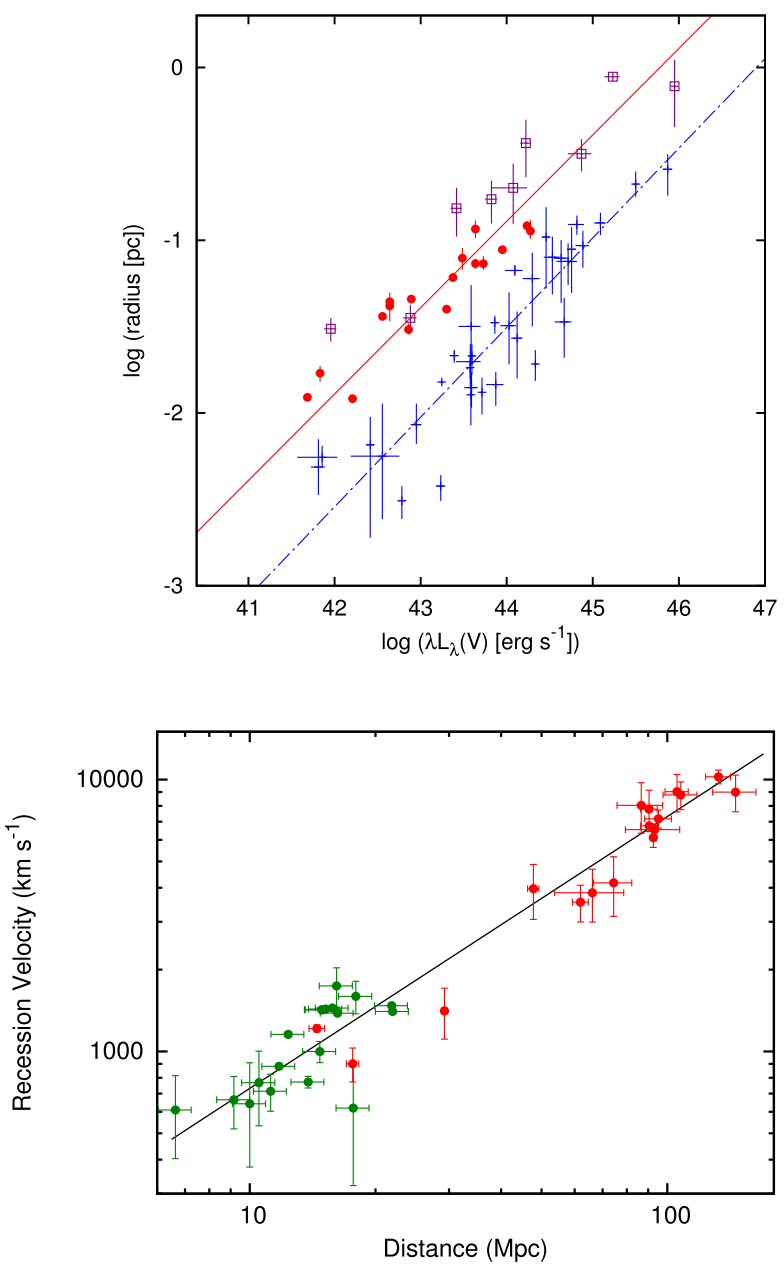

\subsubsection{Trigonometric Parallax for the Dust Torus}

When both the physical size and the angular size of a certain structure in an AGN can be obtained, the angular diameter distance can be estimated by trigonometry. Although usual imaging observations cannot resolve the inner structures of AGNs whose physical sizes are obtained by reverberation mapping, their angular size might be able to be obtained by future long-baseline interferometers in optical and near-infrared wavelengths. This trigonometric parallax for AGNs was first proposed for the BLR (Elvis and Karovska 2002), but actually, it has not been applied to any AGNs yet.

Since the dust torus is more extended than the BLR, the trigonometric parallax for the dust torus would be more feasible. Indeed, recent advancement of near-infrared interferometry makes it possible to measure the angular scale of the innermost dust torus for some brightest AGNs (Kishimoto et al. 2011; Weigelt et al. 2012), and the angular diameter distance of NGC 4151 was estimated by comparing the dust reverberation radius with the interferometric angular scale in near-infrared (Hönig et al. 2014). However, as shown in Fig. 9, the interferometric radius was found to be systematically larger than the reverberation ra- 
dius by about a factor of two (Koshida et al. 2014), whereas it is not the case for NGC 4151. Further studies on the structure of the dust torus are necessary to understand this systematic difference and to extend the use of the dust parallax distance of AGNs.

\subsection{Single Epoch Methods}

Reverberation methods are time-consuming, and much more efficient alternative is to design the method based just on a single spectrum. There are some options in consideration, but they are still in their infancy.

If Eddington ratio and black hole mass can be derived from some distance-independent measure it would be possible to derive distance-independent quasar luminosities. Sulentic et al. (2014) suggest that quasars radiating close to the Eddington limit show distinct optical and UV spectral properties that can be recognized in spectra. They propose specific criteria based on the line ratios (Al III $\lambda 1860 / \mathrm{Si}$ III] $\lambda 1892 \geq 0.5$, and (ii) Si III] $\lambda 1892 / \mathrm{C} \mathrm{III]} \lambda 1909 \geq$ 1.0) which lead to a source sample with dispersion of $\sim 0.13$ dex in the Eddington ratio. Larger sample of such sources may give interesting constraints on cosmological parameters.

Wang et al. (2014) proposed instead to use the basic prediction of the slim accretion disk theory (Abramowicz et al. 1988): the saturation of the luminosity for high Eddington ratio sources. In slim disks the radiative efficiency drops with the Eddington ratio, so effectively the rise of the total source luminosity is only logarithmic in accretion rate. Their preliminary tests of cosmological applications are attractive but the method still requires better analysis of the saturation level as well as efficient method of selecting such AGN in large AGN catalogs.

\subsection{Continuum Shape Method}

Quasars can be considered a reliable cosmological tools in a similar way as for Type Ia supernovae, provided that the quasar sample is large enough and there is a relation able to standardize the quasar emission. A non-linear relation between the optical and X-ray luminosity in quasars was discovered with the first X-ray surveys in the early '80s (Tananbaum et al. 1979; Zamorani et al. 1981; Avni and Tananbaum 1986), and it has been confirmed since with various samples of a few hundred quasars observed with the main X-ray observatories over a redshift range from 0 to $\sim 6.5$ and about four decades in optical luminosity.

The largest samples published so far include (i) a compilation of 367 quasars from different optical surveys and observed by ROSAT, XMM-Newton and Chandra (333 from Steffen et al. 2006 and 34 from Just et al. 2007); (ii) 350 sources obtained from the Sloan Digital Sky Survey $5^{\text {th }}$ quasar catalog with available XMM-Newton observations (Young et al. 2010); (iii) 545 objects from the XMM-COSMOS survey (Lusso et al. 2010); and (iv) 200 quasars with UV and X-ray observations from the Swift observatory archive (Grupe et al. 2010; Vagnetti et al. 2010). In all these works the $L_{X}-L_{U V}$ relation is parametrized as a linear dependence between the logarithm of the monochromatic luminosity at $2500 \AA\left(L_{\mathrm{UV}}\right)$ and the $\alpha_{\mathrm{OX}}$ parameter, defined as the slope of a power law connecting the monochromatic luminosity at $2 \mathrm{keV}\left(L_{\mathrm{X}}\right)$, and $L_{\mathrm{UV}}: \alpha_{\mathrm{OX}}=0.384 \times \log \left(L_{\mathrm{X}} / L_{\mathrm{UV}}\right)$. This relation implies that quasars more luminous in the optical are relatively less luminous in the X-rays. For instance, the increase by a factor of 10 in $L_{\mathrm{UV}}$ implies an increase by only a factor of $\sim 4$ in the X-ray luminosity. When expressed as a relation between X-ray and UV luminosities, the $\alpha_{\mathrm{OX}}-L_{\mathrm{UV}}$ relation becomes $\log L_{\mathrm{X}}=\beta+\alpha \log L_{\mathrm{UV}}$. All the works cited above provide consistent values (within the uncertainties) of both normalization and slope, $\beta \sim 8-9$ and $\alpha \sim 0.6$, with an observed dispersion that ranges between $\delta \sim 0.35-0.40$ dex. Luminosities 
are derived from fluxes through a luminosity distance $\left(D_{\mathrm{L}}\right)$ calculated assuming a standard $\Lambda \mathrm{CDM}$ model with the best estimates of the cosmological parameters $\Omega_{\mathrm{M}}$ and $\Omega_{\Lambda}$ at the time of the publications.

The potential use of this relation as a cosmological probe is obvious. The observed X-ray flux is a function of the observed optical flux, the redshift, and the parameters of the adopted cosmological model as

$$
\log \left(F_{\mathrm{X}}\right)=\Phi\left(F_{\mathrm{UV}}, D_{\mathrm{L}}\right)=\beta^{\prime}+\alpha \log \left(F_{\mathrm{UV}}\right)+2(\alpha-1) \log \left(D_{\mathrm{L}}\right)
$$

where $\beta^{\prime}=\beta+(\alpha-1) \log (4 \pi)$. This relation can be then fitted to a set of optical and X-ray observations of quasars in order to estimate the cosmological parameters. However, none of the samples published so far had, on their own, the size and/or homogeneity to provide useful constraints for cosmological applications.

Recently, the relation above has been employed to build a Hubble diagram for quasars to obtain an independent measure of cosmological parameters. Risaliti and Lusso (2015) have shown that it is possible to build a sample large enough to estimate the cosmological parameters and to test the $\Lambda \mathrm{CDM}$ model over the whole redshift range $z=0-6.5$ (back to an age of the Universe of only $\sim 0.8 \mathrm{Gyr}$ ). By using a dataset of $\sim 800$ quasars from the literature (basically all the ones listed at the beginning of this Section) with optical and Xray data, Risaliti and Lusso (2015) have demonstrated that the $F_{\mathrm{X}}-F_{\mathrm{UV}}$ relation does not exhibit any dependence with redshift, which is a necessary condition to use it as a distance estimator. It has been therefore possible to build, for the first time, a quasar Hubble diagram in excellent agreement with that of Type Ia supernovae over the common redshift range $(z \leq 1.4)$, but extending it to much higher distances. Figure 11 (left panel) shows the Hubble diagram for quasars (small black points, while red points are averages in narrow redshift bins for visual purpose only), compared to the one of Type Ia supernovae samples (cyan points, from Suzuki et al. 2012). Interestingly, since the same physical quantity (i.e. the distance modulus) is involved, this analysis based on the distance modulus-redshift relation allows us to directly merge quasar and supernova data on the same diagram, where both are used as standardized candles. This method offers unique access to a region of the distance modulus-redshift plane where it is extremely unlikely (if not impossible) to observe any Type Ia supernovae.

The results for the determination of the cosmological parameters $\Omega_{\mathrm{M}}$ and $\Omega_{\Lambda}$ are shown in the right panel of Fig. 11. The $68 \%$ and $95 \%$ contours for $\Omega_{\mathrm{M}}$ and $\Omega_{\Lambda}$, assuming a standard $\Lambda \mathrm{CDM}$ model, are derived from the analysis of the Hubble diagram of quasars only (grey contours), from the supernovae of the Union 2.1 sample (empty black contours; Suzuki et al. 2012), and from a quasars-supernovae joint fit (orange/red contours). The present data return $\Omega_{\mathrm{M}}=0.22_{-0.10}^{+0.08}$ and $\Omega_{\Lambda}=0.92_{-0.30}^{+0.18}\left(\Omega_{\mathrm{M}}=0.28 \pm 0.04\right.$ and $\Omega_{\Lambda}=0.73 \pm 0.08$ from a joint fit). The contour plot relative to the joint sample is not a statistical intersection of the two individual contours, but the result of a simultaneous fit of the distance modulus-redshift relation for the combined sample quasar/supernovae. This also provides a significantly improved measurement of $\Omega_{\mathrm{M}}$ and $\Omega_{\Lambda}$ with pure distance indicators. When all the available cosmological indicators (CMB, BAO, weak lensing, supernovae) are considered, the sample of Risaliti and Lusso (2015) does not significantly improve the constraints on the cosmological parameters. The main limitation of this work is its small predictive power, mainly due to the still large dispersion of the $\log F_{\mathrm{X}}=\beta+\alpha \log F_{\mathrm{UV}}$ relation $(\sim 0.30-0.35 \mathrm{dex})$. For comparison, the uncertainty in the distance of a supernova at low redshift is about 0.05 in the same units. This means that, in order to obtain the same constraints on a cosmological fit, roughly $(0.35 / 0.05)^{2} \sim 50$ quasars per single supernova are needed. 


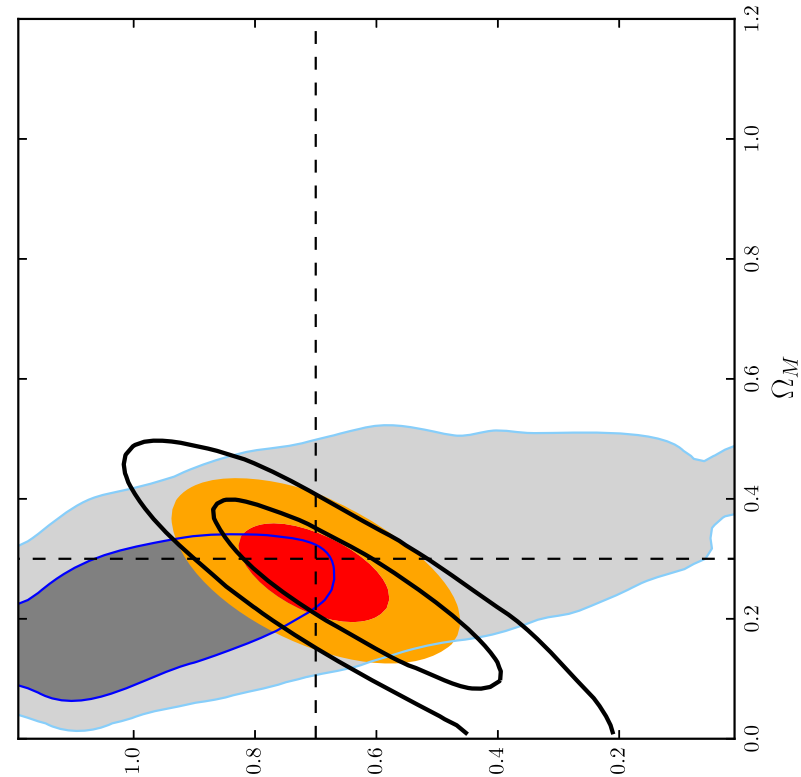

vis

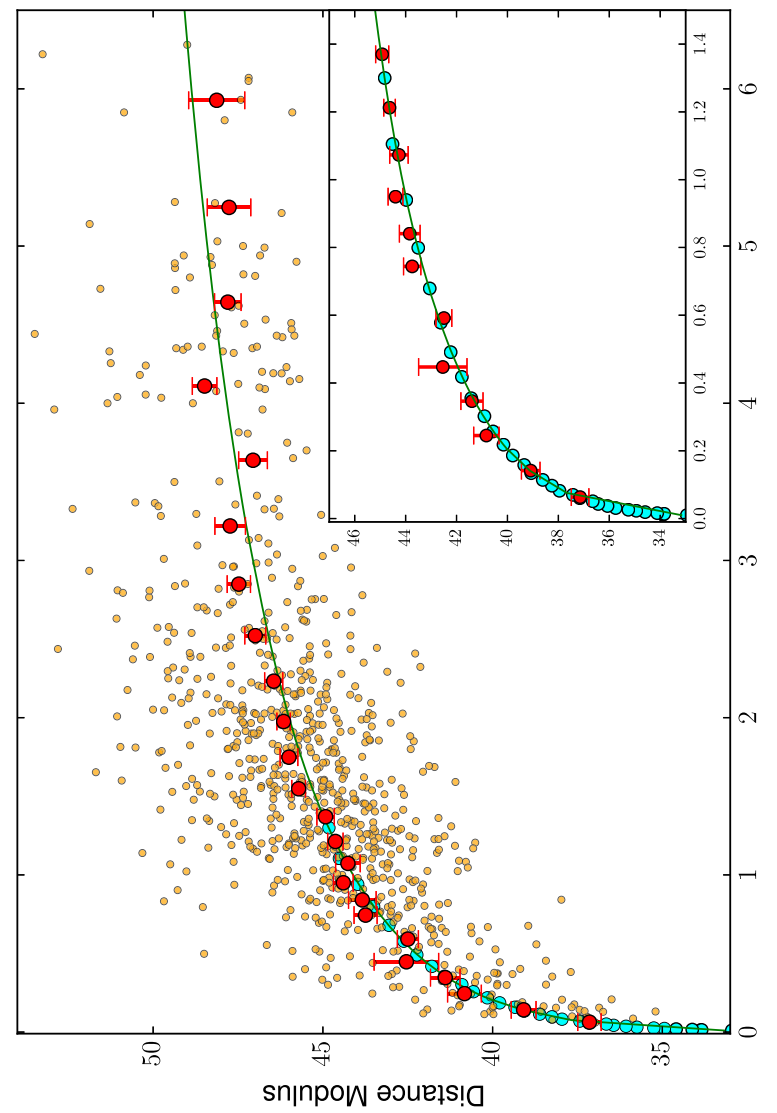

范些

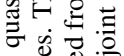

흘

홍 ฮ

范

₹ $\bar{\sigma}$

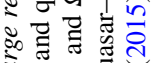

ङ场

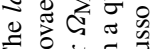

总

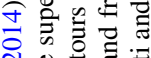

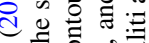

बं

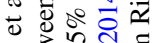

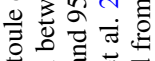

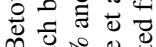

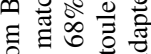

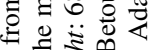

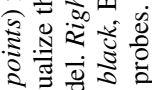

5.

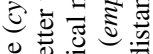

\% 80.7

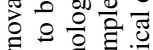

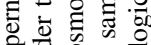

部

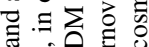

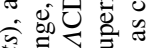

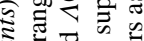

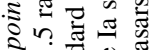

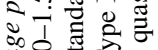

5 에

$\sigma_{N}$ o 00

งิ

它语

o :

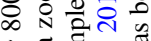

뉴요

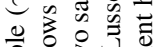

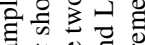

范导

눙

品范

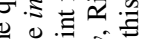

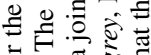

可产焉

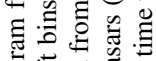

50 氜

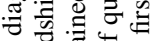

윽 운

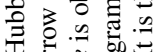

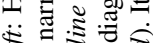

ง. $\cong \cong \cong$

$\Rightarrow$ 要

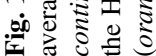


The $L_{\mathrm{X}}-L_{\mathrm{UV}}$ relation was recently analyzed on a sample of $>2,600$ SDSS AGN, with $\mathrm{X}$-ray data from XMM-Newton with the aim to understand the origin of the observed dispersion, and to evaluate the true intrinsic dispersion in this relation (Lusso and Risaliti 2016). This work shows that, even though the observed dispersion in the main quasar sample is $\sim 0.45$ dex, most of this scatter is caused by the combination of X-ray variability, poor opti$\mathrm{cal} / \mathrm{UV}$ and X-ray data quality, and the contamination from the host galaxy and/or AGN with red continua. Once all the contaminations above are taken into account, the real physical dispersion is found to be less than 0.21 dex over roughly 4 decades in luminosity, indicating a tight empirical coupling between disc (UV) and corona (X-rays), which puts the determination of distances based on this relation on a sounder physical grounds.

Although exploratory, the analysis presented in Risaliti and Lusso (2015) already provides important results: the validation of the UV to X-ray relation and the extension of the Hubble Diagram up to $z \sim 6$. The constraints on the cosmological parameters are still loose compared with other methods, and the improvement on the measurement errors obtained by combining the quasar findings with those from supernovae, CMB, BAOs, and clusters is not significant. This work is mainly intended as a demonstration of the method, while most of its potential is still to be exploited in future work, with both available and forthcoming data (e.g. eROSITA, Euclid, Athena) that will significantly increase the number of quasars at $z>2$ where the intrinsic power of this novel technique lies.

\subsection{X-Ray Excess Variance Method}

Another method based on a combination of X-ray observations has been proposed by La Franca et al. (2014). The method requires a single optical spectrum (determination of FWHM of one of the strong AGN lines, preferentially $\mathrm{H} \beta$ ) and determination of the X-ray excess variance. The basic idea is simple, it relies on a combination of two methods of the black hole mass determination and subsequent elimination of the mass. The first method, derived from the reverberation campaigns (e.g., Kaspi et al. 2000; Peterson et al. 2004) shows that the black hole mass can be determined from a single epoch spectrum, from the monochromatic absolute luminosity (a proxy for the BLR size, see Sect. 6.3) and the emission line width. The second method is based on the simple relation between the black hole mass and the high frequency tail of the X-ray power spectrum, or, more conveniently, the Xray excess variance (e.g., Nikolajuk et al. 2004; Gierliński et al. 2008). Combining the two and eliminating black hole mass one can determine the absolute luminosity from the X-ray excess variance and the FWHM. The difficulty lies in the high quality measurement of the X-ray excess variance: La Franca et al. argue that the launch of Athena X-ray Observatory (expected in 2028) will provide interesting number of measurements, and they expect more from the future generation X-ray telescopes with larger detector area, but this is not coming soon.

\section{Galaxy Cluster Distance via X-Ray and Sunyaev-Zel'dovich Effect}

Galaxy clusters are the largest virialized astronomical structures in the Universe and observations of their physical properties can provide important cosmological information. An important phenomenon occurring in galaxy clusters is the Sunyaev-Zel'dovich effect (Sunyaev and Zeldovich 1972; Birkinshaw 1999; Carlstrom et al. 2002), a small distortion of the cosmic microwave background radiation (CMBR) spectrum provoked by the inverse Compton scattering of the CMBR photons passing through a population of hot electrons. The 
Sunyaev-Zel'dovich effect (SZE) is proportional to the electron pressure integrated along the line of sight, i.e., to the first power of the plasma density. The measured temperature decrement $\Delta T_{\mathrm{SZ}}$ of the CMBR is given by (De Filippis et al. 2005)

$$
\frac{\Delta T_{\mathrm{SZ}}}{T_{\mathrm{CMBR}}}=f\left(v, T_{\mathrm{e}}\right) \frac{\sigma_{\mathrm{T}} k_{\mathrm{B}}}{m_{\mathrm{e}} c^{2}} \int_{\text {l.o.s. }} n_{e} T_{\mathrm{e}} d l
$$

where $T_{\mathrm{e}}$ is the temperature of the intra-cluster medium, $k_{\mathrm{B}}$ the Boltzmann constant, $T_{\mathrm{CMBR}}=2.728^{\circ} \mathrm{K}$ is the temperature of the CMBR, $\sigma_{\mathrm{T}}$ the Thompson cross section, $m_{\mathrm{e}}$ the electron mass and $f\left(v, T_{\mathrm{e}}\right)$ accounts for frequency shift and relativistic corrections (Itoh et al. 1998).

Other important physical phenomena occurring in the intra-galaxy cluster medium are the $\mathrm{X}$-ray emission caused by thermal bremsstrahlung and line radiation resulting from electronion collisions. The X-ray surface brightness $S_{X}$ is proportional to the integral along the line of sight of the square of the electron density. This quantity may be written as follows

$$
S_{X}=\frac{D_{A}^{2}}{4 \pi D_{L}^{2}} \int_{\text {l.o.s. }} n_{e}^{2} \Lambda_{e H} d l,
$$

where $\Lambda_{e H}$ is the X-ray cooling function of the intra-cluster medium (measured in the cluster rest frame), $D_{A}$ and $D_{L}$ are the angular diameter and luminosity distances of the galaxy cluster, respectively, and $n_{e}$ is the electron number density. It thus follows that the SZE and X-ray emission both depend on the properties $\left(n_{e}, T_{e}\right)$ of the intra cluster medium.

As is well known, it is possible to obtain the angular diameter distance (ADD) of galaxy clusters by their SZE and X-ray surface brightness observations (SZE/X-ray technique). The calculation begins by constructing a model for the cluster gas distribution. Assuming, for instance, the spherical isothermal $\beta$-model such that $n_{e}$ is given by (Cavaliere and FuscoFemiano 1978)

$$
n_{e}(r)=\left(1+\frac{r^{2}}{r_{c}^{2}}\right)^{-3 \beta / 2},
$$

equations (11) and (12) can be integrated. Here $r_{c}$ is the core radius of the galaxy cluster. This $\beta$ model is based on the hydrostatic equilibrium equation and constant temperature (Sarazin 1988). In this way, we may write for the SZE

$$
\Delta T_{\mathrm{SZ}}=\Delta T_{0}\left(1+\frac{\theta^{2}}{\theta_{c}^{2}}\right)^{1 / 2-3 \beta / 2},
$$

where $\theta_{c}=r_{c} / D_{A}$ is the angular core radius and $\Delta T_{0}$ is the central temperature decrement that includes all physical constants and terms resulting from the line-of-sight integration. More precisely:

$$
\Delta T_{0} \equiv T_{\mathrm{CMBR}} f\left(v, T_{\mathrm{e}}\right) \frac{\sigma_{\mathrm{T}} k_{\mathrm{B}} T_{\mathrm{e}}}{m_{\mathrm{e}} c^{2}} n_{e 0} \sqrt{\pi} \theta_{c} D_{A} g(\beta / 2)
$$

with

$$
g(\alpha) \equiv \frac{\Gamma[3 \alpha-1 / 2]}{\Gamma[3 \alpha]},
$$


where $\Gamma(\alpha)$ is the gamma function and the others constants are the usual physical quantities. For X-ray surface brightness, we have

$$
S_{X}=S_{X 0}\left(1+\frac{\theta}{\theta_{c}^{2}}\right)^{1 / 2-3 \beta},
$$

where the central surface brightness $S_{X 0}$ reads

$$
S_{X 0} \equiv \frac{D_{A}^{2} \Lambda_{e H} \mu_{e} / \mu_{H}}{D_{L}^{2} 4 \sqrt{\pi}} n_{e 0}^{2} \theta_{c} D_{A} g(\beta) .
$$

Here $\mu$ is the molecular weight given by $\mu_{i} \equiv \rho / n_{i} m_{p}$.

One can solve (15) and (18) for the ADD by eliminating $n_{e 0}$ and taking for granted the validity of cosmic distance duality relation (CDDR), $D_{L}(1+z)^{-2} D_{A}^{-1}=\eta=1$. In this case one obtains

$$
\begin{aligned}
D_{A}= & {\left[\frac{\Delta T_{0}^{2}}{S_{\mathrm{X} 0}}\left(\frac{m_{\mathrm{e}} c^{2}}{k_{\mathrm{B}} T_{e 0}}\right)^{2} \frac{g(\beta)}{g(\beta / 2)^{2} \theta_{\mathrm{c}}}\right] } \\
& \times\left[\frac{\Lambda_{e H 0} \mu_{e} / \mu_{H}}{4 \pi^{3 / 2} f\left(v, T_{\mathrm{e}}\right)^{2}\left(T_{\mathrm{CMBR}}\right)^{2} \sigma_{\mathrm{T}}^{2}\left(1+z_{\mathrm{c}}\right)^{4}}\right]
\end{aligned}
$$

where $z_{c}$ is the galaxy cluster redshift. Recently, such a technique has been applied for a fairly large number of clusters (Reese et al. 2002; De Filippis et al. 2005; Bonamente et al. 2006) with systematic and statistical errors around $20 \%$ and $13 \%$, respectively (see Table 3 in Bonamente et al. 2006). The crucial point in the SZE/X-ray technique is the choice of morphology used to describe the galaxy cluster. The standard spherical geometry has been severely questioned, since Chandra and XMM-Newton observations have shown that clusters usually exhibit an elliptical surface brightness (Sereno et al. 2006). The assumed cluster shape can affect considerably the SZE/X-ray distances, and, consequently, the $H_{0}$ estimates and other astrophysical quantities.

\subsection{SZE/X-Ray Technique Applications: the Hubble Constant}

The Hubble constant, $H_{0}$, sets the scale of the size and age of the Universe and its determination from independent methods is still worthwhile to be investigated. Severeal authors used the SZE/X-ray technique to estimate the Hubble constant (see table in Holanda et al. 2012a), however, since the samples used has galaxy clusters in high redshifts (up to $z \approx 0.90$ ), a cosmological model had to be assumed in analysis, usually a flat $\Lambda \mathrm{CDM}$ model. Moreover, due to degeneracy on the cosmological parameters, the matter density parameter, $\Omega_{M}$, was taken as being $\Omega_{M}=0.3$. An interesting method was adopted by Holanda et al. (2012a). In this case, the authors used a sample of 25 ADD of galaxy clusters in redshift range $0.023<z<0.784$ described by an elliptical $\beta$ model (De Filippis et al. 2005) to constrain $H_{0}$ in dark energy models. In order to avoid the use of priors on the cosmological parameters, a joint analysis involving the ADD, the baryon acoustic oscillations (BAO) and the CMBR Shift Parameter signature was proposed. By taking into account the statistical and systematic errors of the SZE/X-ray technique it was obtained for nonflat $\Lambda \mathrm{CDM}$ model $H_{0}=74_{-4.0}^{+5.0} \mathrm{~km} \mathrm{~s}^{-1} \mathrm{Mpc}^{-1}(1 \sigma)$ whereas for a flat universe with constant equation of state parameter it was obtained $H_{0}=72_{-4.0}^{+5.5} \mathrm{~km} \mathrm{~s}^{-1} \mathrm{Mpc}^{-1}(1 \sigma)$. These values are in full agreement with the latest local estimate performed by Riess et al. (2016): $H_{0}=73.24 \pm 1.74 \mathrm{~km} \mathrm{~s}^{-1} \mathrm{Mpc}^{-1}(1 \sigma)$. 

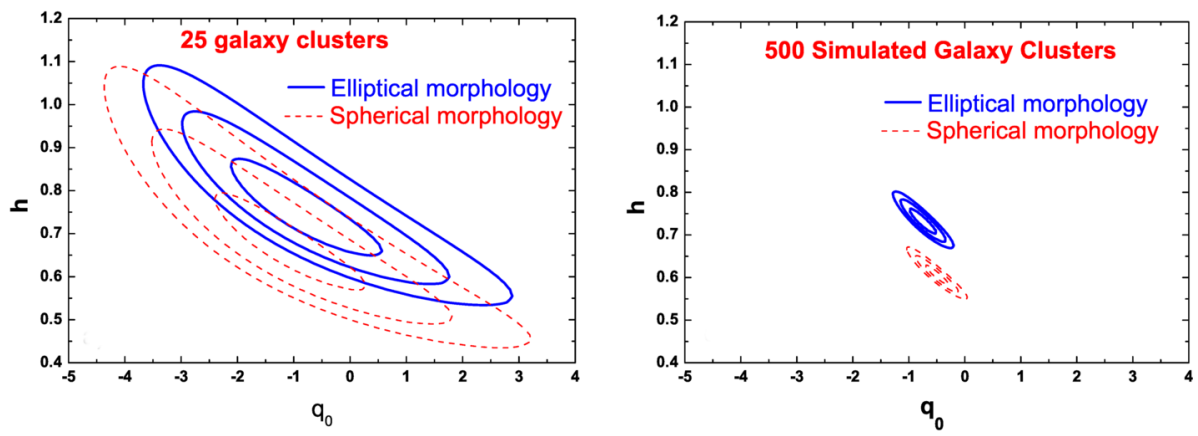

Fig. 12 Confidence contours $(1,2$ and $3 \sigma)$ on the plane $\left(h-q_{0}\right)$. The panel left corresponds to the real data

\subsection{The Search for Two Numbers: $H_{0}$ and $q_{0}$}

A kinematic method to access cosmic acceleration and the $H_{0}$ value based exclusively on the SZE and X-ray surface brightness data from galaxy clusters also was investigated recently. This is a very important task since until very recently the type Ia supernovae observations was the unique direct access to the late time accelerating stage of the Universe. By assuming the current observational error distribution of the samples of 25 ADD from De Filippis et al. (2005), Holanda et al. (2013) performed Monte Carlo simulations based on a well-behaved parametrization for the deceleration parameter, $q_{0}$, to generate samples with different characteristics and study the improvement on the determination of the cosmographic parameters: $q_{0}$ and $H_{0}$. As a interesting result it was shown that, even keeping the current statistical observational uncertainty, an increase in the number of data points increases considerably the figure-of merit for the cosmographic plane $\left(h-q_{0}\right)$, where $h=H_{0} / 100$ (see Fig. 12).

\subsection{The Search for New Physics}

The SZE/X-ray technique also has been shown to being a powerful tool to investigate fundamental physics. Uzan et al. (2004) argued that this technique is strongly dependent on the validity of the CDDR, $D_{L}(1+z)^{-2} D_{A}^{-1}=\eta=1$, valid for all cosmological models based on Riemannian geometry, being dependent neither on Einstein field equations nor on the nature of matter (Etherington 1933 reprinted as Etherington 2007), playing an essential role in modern cosmology. It only requires that source and observer are connected by null geodesics in a Riemannian spacetime and that the number of photons are conserved. However, if $\eta \neq 1$, instead of the real ADD, the measured quantity is $D_{A}^{\text {data }}(z)=D_{A}(z) \eta^{2}(z)$. By using different expressions for $\eta(z)$, several authors have tested the CDDR by using ADD of galaxy clusters and $D_{L}$ from type Ia supernovae observations (Holanda et al. 2010, 2012b; Li et al. 2011b; Nair et al. 2011; Yang et al. 2013; Holanda and Barros 2016). No significant deviation was obtained when an elliptical $\beta$ model was used in analysis (for results from different cosmological observations see Table I in Holanda et al. (2016).

As was showed by Holanda et al. (2016), this technique depends on the fine structure constant, $\alpha$. If $\alpha$ is a time-dependent quantity, e.g., $\alpha=\alpha_{0} \phi(z)$, where $\phi$ is a function of redshift, the current ADD data do not provide the real ADD to the cluster but instead $D_{A}^{\text {data }}(z)=\eta^{2}(z) \phi(z) D_{A}(z)$. Constraints on a possible variation of $\alpha$ for a class of dilaton runaway models was performed considering the sample of $D_{A}^{\text {data }}(z)$ from De Filippis et al. (2005) and estimates of $D_{A}(z)$ from type Ia supernovae observations. It was found no significant indication of variation of $\alpha$ with the present data. 
Finally, it is very important to stress that the SZE/X-ray technique is independent of any calibrator usually adopted in the determinations of the distance scale. The above results, therefore, highlight the cosmological interest in ADD measurements of galaxy clusters.

\section{Gamma-Ray Bursts}

Gamma-rays bursts are the brightest individual sources in the Universe (see e.g., Hjorth and Bloom 2012; Berger 2014, for recent reviews), therefore it is more than natural to attempt to use them in cosmology. Those intense flashes of the gamma-ray emission lasting from milliseconds to several hours are not easily affected by the medium between an observer and the source, so they can be easily detected at very high redshifts. The record holder is the gamma-ray burst GRB 090429B at $z=9.4$ (Cucchiara et al. 2011), with photometric redshift determination, or GRB 090429B at $z=8.2$ (Tanvir et al. 2009), if we limit ourselves to spectroscopically confirmed redshift. Gamma-ray prompted emission is accompanied by afterglow emission at other wavelengths.

However, due to the large burst variety and the critical role of the relativistic boosting of the part of the observed multi-wavelength emission the use of the gamma-ray bursts as distance indicators is rather difficult. The first attempts based on relatively large number of sources was made by Schaefer (2007) who successfully constructed the Hubble diagram in a broad redshift range almost up to $z \sim 7$. The method was based on empirical correlations established for sources at $z<2$ and then extended to higher redshifts.

Several correlations can be used for that purpose (see e.g., Wang et al. 2015). However, there are two basic problems which are not easy to overcome. First, there is some circularity problem, as there are no low redshift gamma-ray bursts and the scaling of the relations has to be done for intermediate redshift sources where cosmological effects already become important. Second, the number of gamma-ray bursts with well measured parameters is still low, and it increases only slowly with time. Samples with high quality data and low scatter in the measured quantities contain less than 50 objects so far (e.g., Dainotti et al. 2016). In addition, the results can be affected by the weak lensing, and the appropriate statistical corrections should be introduced, as discussed by Wang and Dai (2011).

\section{Astronomical Distances from Gravitational-Wave Observations}

Recent direct detections of gravitational waves from merging binary black hole systems at cosmological distances of $z=0.1-0.2$, performed by two Advanced LIGO detectors (Abbott et al. 2016d,c, 2017a), first binary black-hole merger observation with the global network of three detectors of Advanced LIGO and the Advanced Virgo (Abbott et al. 2017d), as well as the first Advanced LIGO and Advanced Virgo detection of a nearby (at a distance of $40 \mathrm{Mpc}$ ) binary neutron-star merger (Abbott et al. 2017e), followed by a short gamma-ray burst (Abbott et al. 2017c) and broad-band electromagnetic emission observational campaign (Abbott et al. 2017f) create unprecedented opportunities for studying the Universe through a novel, never before explored channel of spacetime fluctuations. Gravitational wave astronomy is often compared to 'listening to' rather than 'looking at' the skies. By design which is motivated by the choice of potential sources, the ground-based gravitational wave detectors, kilometer-size laser interferometer antennas of Advanced LIGO (Aasi et al. 2015) and Advanced Virgo (Acernese et al. 2015), are sensitive in the range of frequencies similar to the audible range of human ears - between $10 \mathrm{~Hz}$ and a few $\mathrm{kHz}$. As in the case of an ear, a 
solitary laser interferometric detector is practically omnidirectional (has a poor angular resolution), and has no imaging capabilities. It registers a coherent signal emitted by a bulk movement of large, rapidly-moving masses. Once emitted, gravitational waves are weakly coupled to the surrounding matter and propagate freely without scattering. This has to be contrasted with the electromagnetic emission which originates at the microscopic level, is strongly coupled to the surroundings and often reprocessed; it carries a reliable information from the last scattering surface only. It seems therefore that gravitational wave detectors are the perfect counterpart to the electromagnetic observatories as they may provide us with information impossible to obtain by other means.

Very shortly after announcing the general theory of relativity in 1915, Albert Einstein realized that a linearized version of his equations resembles the wave equation (Einstein 1916). The solution is interpreted as a short-wavelength, time-varying curvature deformation propagating with the speed of light on an otherwise slowly-varying, large-scale curvature background (a gravitational-wave "ripple" propagating through the four-dimensional spacetime); from the point of view of a metric tensor, it represents a small perturbation of a stationary background metric. Linear approximation corresponds to the waves propagating in the farfield limit. By exploiting the gauge freedom of the theory one may show that the solution has features similar to electromagnetic waves: it is a transverse wave which may be polarized (has two independent polarizations). Over the next 40 years, during which Einstein changed his mind to argue against their genuineness, a controversy persisted over the true nature of gravitational waves. Only in the late 50s and early 60s the works of Felix Pirani (1956), Herman Bondi (1957), Ivor Robertson and Andrzej Trautman (1960) convincingly showed that gravitational waves are indeed physical phenomena that carry and deposit energy.

A realistic wave phenomenon (and not, say, a coordinate artifact) must be capable of transmitting energy from the source to infinity. If the amplitude of an exemplary isotropic field at a radial distance $r$ from the source is $h(r)$, then the flux of energy over a spherical surface at $r$ is $\mathcal{F}(r) \propto h^{2}(r)$, and the total emitted power (the luminosity) is $\mathcal{L}(r) \propto 4 \pi r^{2} h^{2}(r)$. Since the energy has to be conserved, the amplitude $h(r)$ falls like $1 / r$, irrespectively of the multipole character of the source (the lowest radiating multipole in the gravity theory is the quadrupole distribution, because for an isolated system a time-changing monopole would correspond to the violation of the mass-energy conservation, and a time-changing dipole would violate the momentum conservation law).

Gravitational waves are related to the changes in the spacetime distance (the proper time interval), therefore they cannot be detected by a local measurement - one has to compare the spacetime positions of remote events (Pirani 1956). The detection principle in the case of the laser interferometric detector is to measure the difference of the relative change in its perpendicular arms' lengths $L_{x}$ and $L_{y}, \delta L_{x}-\delta L_{y}=\Delta L / L$, by measuring the interference pattern in the output port located at the apex of the device. Due to the quadrupolar nature of a gravitational wave, shortening of one arm corresponds to elongation of the other. This change of lengths is reflected in varying paths that the laser light has to cross before the interference. The dimensionless gravitational-wave amplitude $h=\Delta L / L$ (the "strain") is proportional to the amount of outgoing laser light. The fact that the directly-measurable quantity is the amplitude $h \propto 1 / r$, not the energy of the wave as in the electromagnetic antennæ, has a direct consequence for the reach of the observing device: one-order-of-magnitude sensitivity improvement corresponds to one-order-of-magnitude growth of distance reach $r$, as opposed to the factor of $\sqrt{10}$ in the electromagnetic observations (consequently, the volume of space grows like $r^{3}$ in case of gravitational-wave observations, encompassing hundreds of thousands of galaxies for a distance reach of the order of hundreds of Mpc (see Abbott et al. 2016b). 
Among promising sources of gravitational radiation are all asymmetric collapses and explosions (supernovæ), rotating deformed stars (gravitational-wave 'pulsars' of continuous and transient nature), and tight binary systems of e.g., neutron stars and black holes. In the following we will focus on the binary systems, since their properties make them the analogues to standard candles of traditional astronomy. The fittingly descriptive term "standard siren" was first used in the work of Holz and Hughes (2005) in the context of gravitational waves from super-massive binary black holes as a target for the planned spaceborne LISA detector (Kea 1996). Dalal et al. (2006) were the first one to point out that short GRBs produced by merging binary neutron-star systems would constitute ideal standard siren. The idea of using well-understood signals to infer the distance and constrain the cosmological parameters, e.g., the Hubble constant, was however proposed much earlier (Schutz 1986, 2002). Recently it was demonstrated in practice for the first time with the observations of a binary neutron-star merger in both gravitational waves and photons (Abbott et al. 2017e,b).

Magnitudes of the gravitational-wave strain $h$ and the luminosity $\mathcal{L}$ may be estimated using dimensional analysis and Newtonian physics. As the waves are generated by the accelerated movement of masses and the mass distribution should be quadrupolar, one may assume that $h$ is proportional to a second time derivative of the quadrupole moment $I_{i j}=\int \rho(\mathbf{x}) x_{i} x_{j} d^{3} x$ for some matter distribution $\rho(\mathbf{x})$. For a binary composed of masses $m_{1}$ and $m_{2}$, orbiting the center of mass at a separation $a$ with the orbital angular velocity $\omega, h$ is proportional to the system's moment of inertia $\mu a^{2}$ and to $\omega^{2}$, as well as inversely proportional to the distance, $h \propto \mu a^{2} \omega^{2} / r$, where $\mu=m_{1} m_{2} / M$ is the reduced mass, and $M=m_{1}+m_{2}$ the total mass. In order to recover the dimensionless $h$, the characteristic constants of the problem, $G$ and $c$, are used to obtain

$$
h \simeq \frac{G}{c^{4}} \frac{1}{r} \mu a^{2} \omega^{2}=\frac{G^{5 / 3}}{c^{4}} \frac{1}{r} \mu M^{2 / 3} \omega^{2 / 3} \quad\left(h_{i j}=\frac{2 G}{c^{4} r} \ddot{I}_{i j}\right),
$$

with the use of Kepler's third law $\left(G M=a^{3} \omega^{2}\right)$ in the second equation. The expression in brackets represents the strain tensor $h_{i j}$ in the non-relativistic quadrupole approximation (Einstein 1918). Similarly, the luminosity $\mathcal{L}$ (the rate of energy loss in gravitational waves, integrated over a sphere at a distance $r$ ) should be proportional to $h^{2} r^{2}$ and some power of $\omega$. From dimensional analysis one has

$$
\begin{gathered}
\mathcal{L}=\frac{d E_{G W}}{d t} \propto \frac{G}{c^{5}} h^{2} \omega^{2} \propto \frac{G}{c^{5}} \mu^{2} a^{4} \omega^{6} \\
\left(\mathcal{L}=\frac{c^{3}}{16 G \pi} \iint\left\langle\dot{h}_{i j} \dot{h}^{i j}\right\rangle d S=\frac{G}{5 c^{5}}\left\langle\dddot{I}_{i j} \dddot{I}^{i j}\right\rangle\right),
\end{gathered}
$$

with the proportionality factor of 32/5. Again, the expression in brackets refers to the quadrupole approximation; the angle brackets denote averaging over the orbital period. Waves leave the system at the expense of its orbital energy $E_{\text {orb }}=-G m_{1} m_{2} /(2 a)$. Using the time derivative of the Kepler's third law, $\dot{a}=-2 a \dot{\omega} /(3 \omega)$, one gets the evolution of the orbital frequency driven by the gravitational-wave emission:

$$
\frac{d E_{\text {orb }}}{d t} \equiv \frac{G m_{1} m_{2}}{2 a^{2}} \dot{a}=-\frac{d E_{G W}}{d t} \Longrightarrow \dot{\omega}=\frac{96}{5} \frac{\omega^{11 / 3}}{c^{5}} G^{5 / 3} \mathcal{M}^{5 / 3} .
$$

The system changes by increasing its orbital frequency; at the same time the strain amplitude $h$ of emitted waves also grows. This characteristic frequency-amplitude evolution is 
called the chirp, by the similarity to birds' sounds, and the characteristic function of component masses $\mathcal{M}=\left(\mu^{3} M^{2}\right)^{1 / 5}$ is called the chirp mass. Orbital frequency is related in a straightforward manner to the gravitational-wave frequency $f_{G W}$ : from the geometry of the problem it is evident that the frequency of radiation is predominantly at twice the orbital frequency, $f_{G W}=\omega / \pi$. By combining the equations for $\dot{f}_{G W}$ and $h$, one recovers the distance to the source $r$. It is a function of the frequency and amplitude parameters, which are directly measured by the detector:

$$
r=\frac{5}{96 \pi^{2}} \frac{c}{h} \frac{\dot{f}_{G W}}{f_{G W}^{3}}=512 \frac{1}{h_{21}}\left(\frac{0.01 \mathrm{~s}}{\tau}\right)\left(\frac{100 \mathrm{~Hz}}{f_{G W}}\right)^{2} \mathrm{Mpc},
$$

where $h_{21}$ denotes the strain in the units of $10^{-21}$ and $\tau=f_{G W} / \dot{f}_{G W}$ denotes the rate of change of the gravitational-wave chirp frequency. Note that within the simple Newtonian approximation presented here (at the leading order of the post-Newtonian expansion) the product $h \tau$ is independent of the components' masses (Królak and Schutz 1987). The simplified analysis presented above does not take into account the full post-Newtonian waveform, polarization information, network of detectors analysis etc., but is intended to demonstrate that binary systems are indeed truly extraordinary "standard sirens". Their observations provide absolute, physical distances directly, without the need for a calibration or a 'distance ladder'. Among them, binary black hole systems occupy a special position. In the framework of general relativity, binary black holes waveforms are independent from astrophysical assumptions about the systems' intrinsic parameters and their environment. At cosmological scales the distance $r$ has a true meaning of the luminosity distance. However, since the vacuum (black-hole) solutions in general relativity are scale-free, the measurements of their waveforms alone cannot determine the source's redshift. The parameters measured by the detector are related to the rest-frame parameters by the redshift $z: f_{G W}=f_{G W}^{r f} /(1+z)$, $\tau=\tau^{r f}(1+z), \mathcal{M}=\mathcal{M}^{r f}(1+z)$. Independent measurements of the redshift which would facilitate the cosmographic studies of the large-scale Universe requires the collaboration with the electromagnetic observers i.e., the multi-messenger astronomy. This may be obtained by assessing the redshift of the galaxy hosting the binary by detecting the electromagnetic counterpart of the event (Bloom et al. 2009; Singer et al. 2016; Abbott et al. 2017f), or by performing statistical study for galaxies' redshifts correlated with the position of the signal's host galaxy (MacLeod and Hogan 2008). The omnidirectional nature of a solitary detector is mitigated by simultaneous data analysis from at least three ground-based detectors in order to perform the triangulation of the source position, hence the crucial need for the LIGO-Virgo collaboration, which will be in the future enhanced by the LIGO-India detector, and the KAGRA detector in Japan (Aso et al. 2013). The above principles were validated by the observations of a binary black-hole merger with three detector network (Abbott et al. 2017d), resulting in improved distance and sky localization.

Compact binaries containing matter are perfect sources to produce the multi-messenger events. Neutron-star binaries are now firmly established as sources of short gamma-ray bursts (idea originally proposed in Paczynski 1986), thanks to simultaneous gravitational and electromagnetic-wave observations from the same event (Abbott et al. 2017f,c and references therein). Although the complete waveform which includes the merger requires the knowledge of the material properties of the components (the presently poorly-known densematter equation of state physics), the chirp waveforms are understood well enough to facilitate a firm detection and a distance measurement. Binaries involving neutron stars are the ideal "standard sirens" insofar as they naturally provide both loud gravitational-wave and bright electromagnetic emission. Short gamma-ray bursts occur frequently within the reach 
of ground-based detectors, at redshifts $z<0.2$. Nissanke et al. (2013) show that observing a population of the order of 10 of gravitational-wave events related to short gamma-ray bursts would allow to measure the Hubble constant with 5\% precision using a network of detectors that includes advanced LIGO and Virgo (30 beamed events could constrain the Hubble constant to better than $1 \%$ ). In both cases of double black-hole binaries and those involving neutron stars, rapid electromagnetic counterpart observations and precise catalogs of galaxies are needed (Abbott et al. 2016b; Singer et al. 2016).

The main source of error in the distance measurement is the limited sensitivity of the detectors (finite signal-to-noise), which translates to a limited knowledge of the source's direction and orientation (see e.g.,Nissanke et al. 2010 for a short gamma-ray burst related study). This may be improved with the measurements of gravitational-wave polarizations with a network of three or more detectors. Second limiting factor is the detectors' calibration uncertainties. Recent direct detectors of binary black-hole mergers by two Advanced LIGO detectors established the distances with rather large error bars mostly due to these factors (see e.g., Abbott et al. 2016a); the order-of-magnitude improvement in sky localization was evident for the first triple-detection with the global network of Advanced LIGO and Advanced Virgo detectors (Abbott et al. 2017d). Presence of a third detector in the network proved to be crucial for the precise sky localization of a binary neutron-star merger (Abbott et al. 2017f), allowing for rapid electromagnetic follow-up. For redshifts larger than $z=1$ weak gravitational lensing will contribute to the distortion of the luminosity distance measurements at the order of $10 \%$ (Bartelmann and Schneider 2001; Dalal et al. 2006). For a detailed discussion of limiting factors in the case of a network of detectors see Schutz (2011) and references therein.

To conclude, observations of gravitational-waves from cosmological distances with current and planned detectors (e.g., spaceborne LISA, sensitive to low frequencies corresponding to chirping super-massive black hole binaries, Kea 1996, or a third-generation cryogenic underground Einstein Telescope, with an extended low frequency range compared to the Advanced LIGO and Advanced Virgo, Abernathy et al. 2011) promise a wealth of new astrophysical information, as demonstrated by the first multi-messenger gravitational-wave event (Abbott et al. 2017f,c). Future detectors will reach cosmological distances and redshifts of a few, being sensitive to practically all the chirping binaries in their sensitivity band in the Universe and providing precise distance measurements (see e.g., Lang and Hughes 2008). In addition to precisely measuring the Hubble constant, cosmological observations would help determine the distances to galaxies, thus contributing to building the standard 'distance ladder' (calibrating electromagnetic standard candles), establish the distribution of galaxies and voids, characterize the evolution of the dark energy and mass density of the Universe, mass distribution through the gravitational lensing, as well as the chemical evolution effects i.e., establishing the onset of star formation (Królak and Schutz 1987; Sathyaprakash and Schutz 2009). The truly multi-messenger era of astronomy is just beginning.

\section{Conclusions}

The coming years will bring new large data sets at various wavelengths of the electromagnetic spectrum, accompanied by a new window due to the discovery of gravitational waves. Dedicated projects and generally oriented space and ground-based observatories will allow to make the distance measurements with current well developed methods more precise, and the new emerging methods have a chance to reach maturity. Large data sets will push the statistical errors down significantly, so the possible systematic biases will become more 
important. Improvements within a given method will be crucial, but the key tests will lie in cross-check of the results based on independent methods. From this point of view, new emerging methods are as important as the further increase in the already impressive accuracy achieved with the use of SN Ia.

Acknowledgements BCZ was supported by the NCN grant 2015/17/B/ST9/03436 and a Fellowship from the Chinese Academy of Sciences, and acknowledges being a part of the network supported by the COST Action TD1403 Big Data Era in Sky and Earth Observation. MB was partially supported by the NCN grants UMO-2014/14/M/ST9/00707, UMO-2013/01/ASPERA/ST9/00001 and the COST Action MP1304 "NewCompStar". RFIH was supported by Conselho Nacional de Desenvolvimento Cientifico e Tecnologico (CNPq). SWJ acknowledges support from NASA/Keck JPL RSA 1508337 and Department of Energy award DE-SC0011636. JJ gratefully acknowledges the support of NASA grant GO-14219.003-A. E.L. is supported by a European Union COFUND/Durham Junior Research Fellowship (under EU grant agreement no. 609412). MDO acknowledges partial support by the PRIN-INAF 2014, "Transient Universe: unveiling new types of stellar explosions with PESSTO" (P.I.: A. Pastorello). We thank ISSI-BJ for hospitality and an engaging workshop.

Open Access This article is distributed under the terms of the Creative Commons Attribution 4.0 International License (http://creativecommons.org/licenses/by/4.0/), which permits unrestricted use, distribution, and reproduction in any medium, provided you give appropriate credit to the original author(s) and the source, provide a link to the Creative Commons license, and indicate if changes were made.

\section{References}

J. Aasi, B.P. Abbott, R. Abbott, T. Abbott et al., Advanced LIGO. Class. Quantum Gravity 32(7), 074001 (2015). 1411.4547. https://doi.org/10.1088/0264-9381/32/7/074001

B.P. Abbott, R. Abbott, T.D. Abbott, M.R. Abernathy, F. Acernese, K. Ackley, C. Adams, T. Adams, P. Addesso, R.X. Adhikari et al., Properties of the binary black hole merger GW150914. Phys. Rev. Lett. 116(24), 241102 (2016a). 1602.03840. https://doi.org/10.1103/PhysRevLett.116.241102

B.P. Abbott, R. Abbott, T.D. Abbott, M.R. Abernathy, F. Acernese, K. Ackley, C. Adams, T. Adams, P. Addesso, R.X. Adhikari et al., Prospects for observing and localizing gravitational-wave transients with Advanced LIGO and Advanced Virgo. Living Rev. Relativ. 19, 1 (2016b). 1304.0670. https://doi.org/10.1007//rr-2016-1

B.P. Abbott, R. Abbott, T.D. Abbott, M.R. Abernathy et al., GW151226: observation of gravitational waves from a 22-solar-mass binary black hole coalescence. Phys. Rev. Lett. 116(24), 241103 (2016c). 1606.04855. https://doi.org/10.1103/PhysRevLett.116.241103

B.P. Abbott, R. Abbott, T.D. Abbott, M.R. Abernathy et al., Observation of gravitational waves from a binary black hole merger. Phys. Rev. Lett. 116(6), 061102 (2016d). 1602.03837. https://doi.org/10.1103/ PhysRevLett.116.061102

B.P. Abbott, R. Abbott, T.D. Abbott, F. Acernese, K. Ackley, C. Adams, T. Adams, P. Addesso, R.X. Adhikari, V.B. Adya et al., GW170104: observation of a 50-solar-mass binary black hole coalesc ence at redshift 0.2. Phys. Rev. Lett. 118(22), 221101 (2017a). 1706.01812. https://doi.org/10.1103/ PhysRevLett.118.221101

B.P. Abbott, R. Abbott, T.D. Abbott, F. Acernese et al., A gravitational-wave standard siren measurement of the Hubble constant. 1710.05835 (2017b)

B.P. Abbott, R. Abbott, T.D. Abbott, F. Acernese et al., Gravitational waves and gamma-rays from a binary neutron star merger: Gw170817 and grb 170817a. Astrophys. J. Lett. 848(2), L13 (2017c). http://stacks.iop.org/2041-8205/848/i=2/a=L13

B.P. Abbott, R. Abbott, T.D. Abbott, F. Acernese et al., Gw170814: a three-detector observation of gravitational waves from a binary black hole coalescence. Phys. Rev. Lett. 119, 141,101 (2017d). https://doi.org/10.1103/PhysRevLett.119.141101

B.P. Abbott, R. Abbott, T.D. Abbott, F. Acernese et al., Gw170817: observation of gravitational waves from a binary neutron star inspiral. Phys. Rev. Lett. 119, 161,101 (2017e). https://doi.org/10.1103/ PhysRevLett.119.161101

B.P. Abbott, R. Abbott, T.D. Abbott, F. Acernese et al., Multi-messenger observations of a binary neutron star merger. Astrophys. J. Lett. 848(2), L12 (2017f). http://stacks.iop.org/2041-8205/848/i=2/a=L12

M. Abernathy, F. Acernese, A.B.P. Allen et al., Einstein gravitational wave telescope conceptual design study. https://tds.ego-gw.it/ql/?c=7954 (2011) 
M.A. Abramowicz, B. Czerny, J.P. Lasota, E. Szuszkiewicz, Slim accretion disks. Astrophys. J. 332, 646-658 (1988). https://doi.org/10.1086/166683

F. Acernese, M. Agathos, K. Agatsuma, D. Aisa et al., Advanced Virgo: a second-generation interferometric gravitational wave detector. Class. Quantum Gravity 32(2), 024001 (2015). 1408.3978. https://doi.org/10.1088/0264-9381/32/2/024001

R. Amanullah, A. Goobar, J. Johansson, D.P.K. Banerjee, V. Venkataraman, V. Joshi, N.M. Ashok, Y. Cao, M.M. Kasliwal, S.R. Kulkarni, P.E. Nugent, T. Petrushevska, V. Stanishev, The peculiar extinction law of SN 2014J measured with the Hubble space telescope. Astrophys. J. Lett. 788, L21 (2014). 1404.2595. https://doi.org/10.1088/2041-8205/788/2/L21

R. Amanullah, J. Johansson, A. Goobar, R. Ferretti, S. Papadogiannakis, T. Petrushevska, P.J. Brown, Y. Cao, C. Contreras, H. Dahle, N. Elias-Rosa, J.P.U. Fynbo, J. Gorosabel, L. Guaita, L. Hangard, D.A. Howell, E.Y. Hsiao, E. Kankare, M. Kasliwal, G. Leloudas, P. Lundqvist, S. Mattila, P. Nugent, M.M. Phillips, A. Sandberg, V. Stanishev, M. Sullivan, F. Taddia, G. Östlin, S. Asadi, R. Herrero-Illana, J.J. Jensen, K. Karhunen, S. Lazarevic, E. Varenius, P. Santos, S.S. Sridhar, S.H.J. Wallström, J. Wiegert, Diversity in extinction laws of type Ia supernovae measured between 0.2 and $2 \mu \mathrm{m}$. Mon. Not. R. Astron. Soc. 453, 3300-3328 (2015). 1504.02101. https://doi.org/10.1093/mnras/stv1505

J.P. Anderson, S. González-Gaitán, M. Hamuy, C.P. Gutiérrez, M.D. Stritzinger, E.F. Olivares, M.M. Phillips, S. Schulze, R. Antezana, L. Bolt, A. Campillay, S. Castellón, C. Contreras, T. de Jaeger, G. Folatelli, F. Förster, W.L. Freedman, L. González, E. Hsiao, W. Krzemiński, K. Krisciunas, J. Maza, P. McCarthy, N.I. Morrell, S.E. Persson, M. Roth, F. Salgado, N.B. Suntzeff, Characterizing the Vband light-curves of hydrogen-rich type II supernovae. Astrophys. J. 786, 67 (2014). 1403.7091. https://doi.org/10.1088/0004-637X/786/1/67

Y. Andrillat, S. Souffrin, Variations du spectre du noyau de la galaxie de Seyfert NGC 3516. Astrophys. Lett. 1, $111(1968)$

R. Antonucci, Unified models for active galactic nuclei and quasars. Annu. Rev. Astron. Astrophys. 31, 473521 (1993). https://doi.org/10.1146/annurev.aa.31.090193.002353

A.L. Argon, L.J. Greenhill, M.J. Reid, J.M. Moran, E.M.L. Humphreys, Toward a new geometric distance to the active galaxy NGC 4258, I: VLBI monitoring of water maser emission. Astrophys. J. 659, 10401062 (2007). astro-ph/0701396. https://doi.org/10.1086/512718

Y. Aso, Y. Michimura, K. Somiya, M. Ando et al., Interferometer design of the KAGRA gravitational wave detector. Phys. Rev. D 88(4), 043007 (2013). 1306.6747. https://doi.org/10.1103/PhysRevD.88.043007

Y. Avni, H. Tananbaum, X-ray properties of optically selected QSOs. Astrophys. J. 305, 83-99 (1986). https://doi.org/10.1086/164230

W. Baade, F. Zwicky, On super-novae. Proc. Natl. Acad. Sci. 20, 254-259 (1934). https://doi.org/10.1073/ pnas.20.5.254

C. Barbarino, M. Dall'Ora, M.T. Botticella, M. Della Valle, L. Zampieri, J.R. Maund, M.L. Pumo, A. Jerkstrand, S. Benetti, N. Elias-Rosa, M. Fraser, A. Gal-Yam, M. Hamuy, C. Inserra, C. Knapic, A.P. LaCluyze, M. Molinaro, P. Ochner, A. Pastorello, G. Pignata, D.E. Reichart, C. Ries, A. Riffeser, B. Schmidt, M. Schmidt, R. Smareglia, S.J. Smartt, K. Smith, J. Sollerman, M. Sullivan, L. Tomasella, M. Turatto, S. Valenti, O. Yaron, D. Young, SN 2012ec: mass of the progenitor from PESSTO follow-up of the photospheric phase. Mon. Not. R. Astron. Soc. 448, 2312-2331 (2015). 1410.8393. https://doi.org/10.1093/mnras/stv106

E. Baron, P.E. Nugent, D. Branch, P.H. Hauschildt, Type IIP supernovae as cosmological probes: a spectralfitting expanding atmosphere model distance to SN 1999em. Astrophys. J. Lett. 616, L91-L94 (2004). astro-ph/0410153. https://doi.org/10.1086/426506

M. Bartelmann, P. Schneider, Weak gravitational lensing. Phys. Rep. 340, 291-472 (2001). astro-ph/9912508. https://doi.org/10.1016/S0370-1573(00)00082-X

A.J. Barth, V.N. Bennert, G. Canalizo, A.V. Filippenko, E.L. Gates, J.E. Greene, W. Li, M.A. Malkan, A. Pancoast, D.J. Sand, D. Stern, T. Treu, J.H. Woo, R.J. Assef, H.J. Bae, B.J. Brewer, S.B. Cenko, K.I. Clubb, M.C. Cooper, A.M. Diamond-Stanic, K.D. Hiner, S.F. Hönig, E. Hsiao, M.T. Kandrashoff, M.S. Lazarova, A.M. Nierenberg, J. Rex, J.M. Silverman, E.J. Tollerud, J.L. Walsh, The lick AGN monitoring project 2011: spectroscopic campaign and emission-line light curves. Astrophys. J. Suppl. Ser. 217, 26 (2015). 1503.01146. https://doi.org/10.1088/0067-0049/217/2/26

R. Barvainis, Hot dust and the near-infrared bump in the continuum spectra of quasars and active galactic nuclei. Astrophys. J. 320, 537-544 (1987). https://doi.org/10.1086/165571

R.L. Beaton, W.L. Freedman, B.F. Madore, G. Bono, E.K. Carlson, G. Clementini, M.J. Durbin, A. Garofalo, D. Hatt, I.S. Jang, J.A. Kollmeier, M.G. Lee, A.J. Monson, J.A. Rich, V. Scowcroft, M. Seibert, L. Sturch, S.C. Yang, The Carnegie-Chicago Hubble program, I: a new approach to the distance ladder using only distance indicators of population II. 1604.01788 (2016)

M.C. Bentz, AGN reverberation mapping. 1505.04805 (2015) 
M.C. Bentz, B.M. Peterson, H. Netzer, R.W. Pogge, M. Vestergaard, The radius-luminosity relationship for active galactic nuclei: the effect of host-galaxy starlight on luminosity measurements, II: the full sample of reverberation-mapped AGNs. Astrophys. J. 697(160-181), doi10.1088/0004-637X/697/1/160 (2009). 0812.2283

M.C. Bentz, K.D. Denney, C.J. Grier, A.J. Barth, B.M. Peterson, M. Vestergaard, V.N. Bennert, G. Canalizo, G. De Rosa, A.V. Filippenko, E.L. Gates, J.E. Greene, W. Li, M.A. Malkan, R.W. Pogge, D. Stern, T. Treu, J.H. Woo, The low-luminosity end of the radius-luminosity relationship for active galactic nuclei. Astrophys. J. 767, 149 (2013). 1303.1742. https://doi.org/10.1088/0004-637X/767/2/149

M.C. Bentz, M. Batiste, J. Seals, K. Garcia, R. Kuzio de Naray, W. Peters, M.D. Anderson, J. Jones, K. Lester, C. Machuca, A low-mass black hole in the nearby Seyfert galaxy UGC 06728. Astrophys. J. 831(2), doi10.3847/0004-637X/831/1/2 (2016a). 1608.03893

M.C. Bentz, E.M. Cackett, D.M. Crenshaw, K. Horne, R. Street, B. Ou-Yang, A reverberation-based black hole mass for MCG-06-30-15. Astrophys. J. 830, 136 (2016b). 1608.01229. https://doi.org/10.3847/ 0004-637X/830/2/136

E. Berger, Short-duration gamma-ray bursts. Annu. Rev. Astron. Astrophys. 52, 43-105 (2014). 1311.2603. https://doi.org/10.1146/annurev-astro-081913-035926

M. Betoule, R. Kessler, J. Guy, J. Mosher, D. Hardin, R. Biswas, P. Astier, P. El-Hage, M. Konig, S. Kuhlmann, J. Marriner, R. Pain, N. Regnault, C. Balland, B.A. Bassett, P.J. Brown, H. Campbell, R.G. Carlberg, F. Cellier-Holzem, D. Cinabro, A. Conley, C.B. D’Andrea, D.L. DePoy, M. doi, R.S. Ellis, S. Fabbro, A.V. Filippenko, R.J. Foley, J.A. Frieman, D. Fouchez, L. Galbany, A. Goobar, R.R. Gupta, G.J. Hill, R. Hlozek, C.J. Hogan, I.M. Hook, D.A. Howell, S.W. Jha, L. Le Guillou, G. Leloudas, C. Lidman, J.L. Marshall, A. Möller, A.M. Mourão, J. Neveu, R. Nichol, M.D. Olmstead, N. Palanque-Delabrouille, S. Perlmutter, J.L. Prieto, C.J. Pritchet, M. Richmond, A.G. Riess, V. Ruhlmann-Kleider, M. Sako, K. Schahmaneche, D.P. Schneider, M. Smith, J. Sollerman, M. Sullivan, N.A. Walton, C.J. Wheeler, Improved cosmological constraints from a joint analysis of the SDSS-II and SNLS supernova samples. Astron. Astrophys. 568, A22 (2014). 1401.4064 https://doi.org/10.1051/0004-6361/201423413

L. Bildsten, K.J. Shen, N.N. Weinberg, G. Nelemans, Faint thermonuclear supernovae from AM Canum Venaticorum binaries. Astrophys. J. Lett. 662, L95-L98 (2007). astro-ph/0703578. https://doi.org/ $10.1086 / 519489$

M. Birkinshaw, The Sunyaev-Zel'dovich effect. Phys. Rep. 310, 97-195 (1999). astro-ph/9808050. https:// doi.org/10.1016/S0370-1573(98)00080-5

J.P. Blakeslee, Cosmic distances from surface brightness fluctuations, in Advancing the Physics of Cosmic Distances, ed. by R. de Grijs. IAU Symposium, vol. 289 (2013), pp. 304-311. https://doi.org/ 10.1017/S174392131202159X

J.P. Blakeslee, A. Jordán, S. Mei, P. Côté, L. Ferrarese, L. Infante, E.W. Peng, J.L. Tonry, M.J. West, The ACS Fornax cluster survey, V: measurement and recalibration of surface brightness fluctuations and a precise value of the Fornax-Virgo relative distance. Astrophys. J. 694, 556-572 (2009). 0901.1138. https://doi.org/10.1088/0004-637X/694/1/556

R.D. Blandford, C.F. McKee, Reverberation mapping of the emission line regions of Seyfert galaxies and quasars. Astrophys. J. 255, 419-439 (1982). https://doi.org/10.1086/159843

S.I. Blinnikov, A.M. Khokhlov, Development of detonations in degenerate stars. Sov. Astron. Lett. 12, 131134 (1986)

S. Blondin, J.L. Prieto, F. Patat, P. Challis, M. Hicken, R.P. Kirshner, T. Matheson, M. Modjaz, A second case of variable Na I D lines in a highly reddened type Ia supernova. Astrophys. J. 693, 207-215 (2009). 0811.0002. https://doi.org/10.1088/0004-637X/693/1/207

J.S. Bloom, D.E. Holz, S.A. Hughes, K. Menou, A. Adams, S.F. Anderson, A. Becker, G.C. Bower, N. Brandt, B. Cobb, K. Cook, A. Corsi, S. Covino, D. Fox, A. Fruchter, C. Fryer, J. Grindlay, D. Hartmann, Z. Haiman, B. Kocsis, L. Jones, A. Loeb, S. Marka, B. Metzger, E. Nakar, S. Nissanke, D.A. Perley, T. Piran, D. Poznanski, T. Prince, J. Schnittman, A. Soderberg, M. Strauss, P.S. Shawhan, D.H. Shoemaker, J. Sievers, C. Stubbs, G. Tagliaferri, P. Ubertini, P. Wozniak, Astro2010 decadal survey whitepaper: coordinated science in the gravitational and electromagnetic skies. 0902.1527 (2009)

M. Bonamente, M.K. Joy, S.J. LaRoque, J.E. Carlstrom, E.D. Reese, K.S. Dawson, Determination of the cosmic distance scale from Sunyaev-Zel'dovich effect and Chandra X-ray measurements of high-redshift galaxy clusters. Astrophys. J. 647, 25-54 (2006). astro-ph/0512349. https://doi.org/10.1086/505291

H. Bondi, Plane gravitational waves in general relativity. Nature 179, 1072-1073 (1957). https:// doi.org/10.1038/1791072a0

S. Bose, B. Kumar, Distance determination to eight galaxies using expanding photosphere method. Astrophys. J. 782, 98 (2014). 1401.5115. https://doi.org/10.1088/0004-637X/782/2/98

M.C.P. Bours, S. Toonen, G. Nelemans, Single degenerate supernova type Ia progenitors. Studying the influence of different mass retention efficiencies. Astron. Astrophys. 552, A24 (2013). 1302.2629. https://doi.org/10.1051/0004-6361/201220692 
J. Braatz, J. Condon, A. Constantin, F. Gao, J. Greene, L. Hao, C. Henkel, V. Impellizzeri, C.Y. Kuo, E. Litzinger, K.Y. Lo, D. Pesce, M. Reid, J. Wagner, W. Zhao, The Megamaser cosmology project: survey completion. IAU Gen. Assem. 22, 2255730 (2015)

P.J. Brown, P.W.A. Roming, P.A. Milne, The first ten years of Swift supernovae. J. High Energy Astrophys. 7, 111-116 (2015a). 1504.08017. https://doi.org/10.1016/j.jheap.2015.04.007

P.J. Brown, M.T. Smitka, L. Wang, A. Breeveld, M. de Pasquale, D.H. Hartmann, K. Krisciunas, N.P. Kuin, P.A. Milne, M. Page, Swift ultraviolet observations of supernova 2014J in M82: large extinction from interstellar dust. Astrophys. J. 805, 74 (2015b). 1408.2381. https://doi.org/10.1088/0004-637X/805/1/74

C.R. Burns, M. Stritzinger, M.M. Phillips, S. Kattner, S.E. Persson, B.F. Madore, W.L. Freedman, L. Boldt, A. Campillay, C. Contreras, G. Folatelli, S. Gonzalez, W. Krzeminski, N. Morrell, F. Salgado, N.B. Suntzeff, The Carnegie supernova project: light-curve fitting with SNooPy. Astron. J. 141, 19 (2011). 1010.4040. https://doi.org/10.1088/0004-6256/141/1/19

C.R. Burns, M. Stritzinger, M.M. Phillips, E.Y. Hsiao, C. Contreras, S.E. Persson, G. Folatelli, L. Boldt, A. Campillay, S. Castellón, W.L. Freedman, B.F. Madore, N. Morrell, F. Salgado, N.B. Suntzeff, The Carnegie supernova project: intrinsic colors of type Ia supernovae. Astrophys. J. 789, 32 (2014). 1405.3934. https://doi.org/10.1088/0004-637X/789/1/32

E.M. Cackett, K. Horne, H. Winkler, Testing thermal reprocessing in active galactic nuclei accretion discs. Mon. Not. R. Astron. Soc. 380, 669-682 (2007). 0706.1464. https://doi.org/10.1111/j.1365-2966. 2007.12098.x

E. Cappellaro, M. Riello, G. Altavilla, M.T. Botticella, S. Benetti, A. Clocchiatti, J.I. Danziger, P. Mazzali, A. Pastorello, F. Patat, M. Salvo, M. Turatto, S. Valenti, Death rate of massive stars at redshift about 0.3. Astron. Astrophys. 430, 83-93 (2005). astro-ph/0407216. https://doi.org/10.1051/0004-6361:20041256

J.A. Cardelli, G.C. Clayton, J.S. Mathis, The relationship between infrared, optical, and ultraviolet extinction. Astrophys. J. 345, 245-256 (1989). https://doi.org/10.1086/167900

J.E. Carlstrom, G.P. Holder, E.D. Reese, Cosmology with the Sunyaev-Zel'dovich effect. Annu. Rev. Astron. Astrophys. 40, 643-680 (2002). astro-ph/0208192. https://doi.org/10.1146/annurev.astro.40.060401. 093803

A. Cavaliere, R. Fusco-Femiano, The distribution of hot gas in clusters of galaxies. Astron. Astrophys. 70, 677 (1978)

D. Chelouche, S. Zucker, Quasar cartography: from black hole to broad-line region scales. Astrophys. J. 769, 124 (2013). 1305.6499. https://doi.org/10.1088/0004-637X/769/2/124

D. Chelouche, S.E. Rafter, G.I. Cotlier, S. Kaspi, A.J. Barth, A tentative size-luminosity relation for the iron emission-line region in quasars. Astrophys. J. Lett. 783, L34 (2014). 1404.6142. https://doi.org/ 10.1088/2041-8205/783/2/L34

A.M. Cherepashchuk, V.M. Lyutyi, Rapid variations of $\mathrm{H} \alpha$ intensity in the nuclei of Seyfert galaxies NGC 4151, 3516, 1068. Astrophys. Lett. 13, 165 (1973)

M. Chevallier, D. Polarski, Accelerating universes with scaling dark matter. Int. J. Mod. Phys. D 10, $213-223$ (2001). gr-qc/0009008. https://doi.org/10.1142/S0218271801000822

L. Chomiuk, SN 2011fe: a laboratory for testing models of type Ia supernovae. Publ. Astron. Soc. Aust. 30, e046 (2013). 1307.2721. https://doi.org/10.1017/pasa.2013.24

N. Chotard, E. Gangler, G. Aldering, P. Antilogus, C. Aragon, S. Bailey, C. Baltay, S. Bongard, C. Buton, A. Canto, M. Childress, Y. Copin, H.K. Fakhouri, E.Y. Hsiao, M. Kerschhaggl, M. Kowalski, S. Loken, P. Nugent, K. Paech, R. Pain, E. Pecontal, R. Pereira, S. Perlmutter, D. Rabinowitz, K. Runge, R. Scalzo, G. Smadja, C. Tao, R.C. Thomas, B.A. Weaver, C. Wu, Nearby Supernova Factory, The reddening law of type Ia supernovae: separating intrinsic variability from dust using equivalent widths. Astron. Astrophys. 529, L4 (2011). 1103.5300. https://doi.org/10.1051/0004-6361/201116723

J.S.W. Claeys, O.R. Pols, R.G. Izzard, J. Vink, F.W.M. Verbunt, Theoretical uncertainties of the type Ia supernova rate. Astron. Astrophys. 563, A83 (2014). 1401.2895. https://doi.org/10.1051/0004-6361/ 201322714

C. Gea, Gaia data release 1. Testing parallaxes with local Cepheids and RR Lyrae stars. Astron. Astrophys. 605, A79 (2017). 1705.00688. https://doi.org/10.1051/0004-6361/201629925

A.D. Code, Hubble's study of the stellar contnet of M31. Astrophys. J. 525, 212 (1999)

S. Collier, K. Horne, I. Wanders, B.M. Peterson, A new direct method for measuring the Hubble constant from reverberating accretion discs in active galaxies. Mon. Not. R. Astron. Soc. 302, L24-L28 (1999). astro-ph/9811278. https://doi.org/10.1046/j.1365-8711.1999.02250.x

S. Collier, D.M. Crenshaw, B.M. Peterson et al., Multiwavelength monitoring of the narrow-line Seyfert 1 galaxy Arakelian 564, II: ultraviolet continuum and emission-line variability. Astrophys. J. 561, 146161 (2001). astro-ph/0107059. https://doi.org/10.1086/323234

S.J. Collier, K. Horne, S. Kaspi et al., Steps toward determination of the size and structure of the broad-line region in active galactic nuclei, XIV: intensive optical spectrophotometric observations of NGC 7469. Astrophys. J. 500, 162-172 (1998). https://doi.org/10.1086/305720 
A. Conley, R.G. Carlberg, J. Guy, D.A. Howell, S. Jha, A.G. Riess, M. Sullivan, Is there evidence for a Hubble bubble? The nature of type Ia supernova colors and dust in external galaxies. Astrophys. J. Lett. 664, L13-L16 (2007). 0705.0367. https://doi.org/10.1086/520625

A. Conley, M. Sullivan, E.Y. Hsiao, J. Guy, P. Astier, D. Balam, C. Balland, S. Basa, R.G. Carlberg, D. Fouchez, D. Hardin, D.A. Howell, I.M. Hook, R. Pain, K. Perrett, C.J. Pritchet, N. Regnault, SiFTO: an empirical method for fitting SN Ia light curves. Astrophys. J. 681, $482-498$ (2008). 0803.3441. https://doi.org/10.1086/588518

L. Cortese, L.M.R. Fogarty, K. Bekki, J. van de Sande, W. Couch, B. Catinella, M. Colless, D. Obreschkow, D. Taranu, E. Tescari, D. Barat, J. Bland-Hawthorn, J. Bloom, J.J. Bryant, M. Cluver, S.M. Croom, M.J. Dreinkwater, F. d'Eugenio, I.S. Konstantopoulos, A. Lopez-Sanchez, S. Mahajan, N. Scott, C. Tonini, O.I. Wong, J.T. Allen, S. Brough, M. Goodwin, A.W. Green, I.T. Ho, L.S. Kelvin, J.S. Lawrence, N.P.F. Lorente, A.M. Medling, M.S. Owers, S. Richards, R. Sharp, S.M. Sweet, The SAMI galaxy survey: the link between angular momentum and optical morphology. Mon. Not. R. Astron. Soc. 463, 170-184 (2016). 1608.00291. https://doi.org/10.1093/mnras/stw1891

D.A. Coulter, C.D. Kilpatrick, R.J. Foley, J. Anais, M.M. Phillips, M.R. Drout, B.F. Madore, A.L. Piro, B.J. Shappee, E.Y. Hsiao, Swope photometric observations of SN 2017cbv = DLT17u. Astron. Telegr. 10167, 167 (2017)

A.P.S. Crotts, Light echoes from supernova 2014J in M82. Astrophys. J. Lett. 804, L37 (2015). 1409.8671. https://doi.org/10.1088/2041-8205/804/2/L37

A.P.S. Crotts, D. Yourdon, The nature and geometry of the light echo from SN 2006X. Astrophys. J. 689, 1186-1190 (2008). 0804.2030. https://doi.org/10.1086/592318

A. Cucchiara, A.J. Levan, D.B. Fox, N.R. Tanvir, T.N. Ukwatta, E. Berger, T. Krühler, A. Küpcü Yoldaş, X.F. Wu, K. Toma, J. Greiner, F.E. Olivares, A. Rowlinson, L. Amati, T. Sakamoto, K. Roth, A. Stephens, A. Fritz, J.P.U. Fynbo, J. Hjorth, D. Malesani, P. Jakobsson, K. Wiersema, P.T. O’Brien, A.M. Soderberg, R.J. Foley, A.S. Fruchter, J. Rhoads, R.E. Rutledge, B.P. Schmidt, M.A. Dopita, P. Podsiadlowski, R. Willingale, C. Wolf, S.R. Kulkarni, P. D’Avanzo, A photometric redshift of $z \sim 9.4$ for GRB 090429B. Astrophys. J. 736, 7 (2011). 1105.4915. https://doi.org/10.1088/0004-637X/736/1/7

B. Czerny, K. Hryniewicz, The origin of the broad line region in active galactic nuclei. Astron. Astrophys. 525, L8 (2011). 1010.6201. https://doi.org/10.1051/0004-6361/201016025

B. Czerny, K. Hryniewicz, I. Maity, A. Schwarzenberg-Czerny, P.T. Zycki, M. Bilicki, Towards equation of state of dark energy from quasar monitoring: reverberation strategy. Astron. Astrophys. 556, A97 (2013). 1212.0472. https://doi.org/10.1051/0004-6361/201220832

E. da Cunha, A.M. Hopkins, M. Colless, E.N. Taylor, C. Blake, C. Howlett, C. Magoulas, J.R. Lucey, C. Lagos, K. Kuehn, Y. Gordon, D. Barat, F. Bian, C. Wolf, M.J. Cowley, M. White, I. Achitouv, M. Bilicki, J. Bland-Hawthorn, K. Bolejko, M.J.I. Brown, R. Brown, J. Bryant, S. Croom, T.M. Davis, S.P. Driver, M.D. Filipovic, S.R. Hinton, M. Johnston-Hollitt, D.H. Jones, B. Koribalski, D. Kleiner, J. Lawrence, N. Lorente, J. Mould, M.S. Owers, K. Pimbblet, C.G. Tinney, N.F.H. Tothill, F. Watson, The Taipan galaxy survey: scientific goals and observing strategy. Publ. Astron. Soc. Aust. 34, e047 (2017). 1706.01246. https://doi.org/10.1017/pasa.2017.41

M.G. Dainotti, S. Postnikov, X. Hernandez, M. Ostrowski, A fundamental plane for long gamma-ray bursts with X-ray plateaus. Astrophys. J. Lett. 825, L20 (2016). 1604.06840. https://doi.org/10.3847/ 2041-8205/825/2/L20

N. Dalal, D.E. Holz, S.A. Hughes, B. Jain, Short grb and binary black hole standard sirens as a probe of dark energy. Phys. Rev. D 74, 063006 (2006). astro-ph/0601275. https://doi.org/10.1103/ PhysRevD.74.063006

M. Dall'Ora, M.T. Botticella, M.L. Pumo, L. Zampieri, L. Tomasella, G. Pignata, The type IIP supernova 2012aw in M95: hydrodynamical modeling of the photospheric phase from accurate spectrophotometric monitoring. Astrophys. J. 787, 139 (2014). 1404.1294. https://doi.org/10.1088/0004-637X/787/2/139

C.B. D’Andrea, M. Sako, B. Dilday, J.A. Frieman, J. Holtzman, R. Kessler, K. Konishi, D.P. Schneider, J. Sollerman, J.C. Wheeler, N. Yasuda, D. Cinabro, S. Jha, R.C. Nichol, H. Lampeitl, M. Smith, D.W. Atlee, B. Bassett, F.J. Castander, A. Goobar, R. Miquel, J. Nordin, L. Östman, J.L. Prieto, R. Quimby, A.G. Riess, M. Stritzinger, Type II-P supernovae from the SDSS-II supernova survey and the standardized candle method. Astrophys. J. 708, 661-674 (2010). 0910.5597. https://doi.org/ 10.1088/0004-637X/708/1/661

D. Kea, Lisa: laser interferometer space antenna for gravitational wave measurements. Class. Quantum Gravity 13, A247-A250 (1996). https://doi.org/10.1088/0264-9381/13/11A/033

T.A. Davis, J. Greene, C.P. Ma, V. Pandya, J.P. Blakeslee, N. McConnell, J. Thomas, The MASSIVE survey, III: molecular gas and a broken Tully-Fisher relation in the most massive early-type galaxies. Mon. Not. R. Astron. Soc. 455, 214-226 (2016). 1510.00729. https://doi.org/10.1093/mnras/stv2313 
E. De Filippis, M. Sereno, M.W. Bautz, G. Longo, Measuring the three-dimensional structure of galaxy clusters, I: application to a sample of 25 clusters. Astrophys. J. 625, 108-120 (2005). astro-ph/0502153. https://doi.org/10.1086/429401

T. de Jaeger, S. González-Gaitán, J.P. Anderson, L. Galbany, M. Hamuy, M.M. Phillips, M.D. Stritzinger, C.P. Gutiérrez, L. Bolt, C.R. Burns, A. Campillay, S. Castellón, C. Contreras, G. Folatelli, W.L. Freedman, E.Y. Hsiao, K. Krisciunas, W. Krzeminski, H. Kuncarayakti, N. Morrell, E.F. Olivares, S.E. Persson, N. Suntzeff, A Hubble diagram from type II supernovae based solely on photometry: the photometric color method. Astrophys. J. 815, 121 (2015). 1511.05145. https://doi.org/10.1088/0004-637X/815/2/121

T. de Jaeger, S. González-Gaitán, M. Hamuy, L. Galbany, J.P. Anderson, M.M. Phillips, M.D. Stritzinger, R.G. Carlberg, M. Sullivan, C.P. Gutiérrez, I.M. Hook, D.A. Howell, E.Y. Hsiao, H. Kuncarayakti, V. Ruhlmann-Kleider, G. Folatelli, C. Pritchet, S. Basa, A type II supernova Hubble diagram from the CSP-I, SDSS-II, and SNLS surveys. Astrophys. J. 835, 166 (2017). 1612.05636. https://doi.org/10.3847/ $1538-4357 / 835 / 2 / 166$

M. della Valle, N. Panagia, Type IA supernovae in late type galaxies-reddening correction, scale height, and absolute maximum magnitude. Astron. J. 104, 696-703 (1992). https://doi.org/10.1086/116265

S. Dhawan, S.W. Jha, B. Leibundgut, Measuring the Hubble constant with type Ia supernovae as near-infrared standard candles. 1707.00715 (2017)

R. Di Stefano, The progenitors of type Ia supernovae, I: are they supersoft sources? Astrophys. J. 712, 728733 (2010). 0912.0757. https://doi.org/10.1088/0004-637X/712/1/728

R. Di Stefano, R. Voss, J.S.W. Claeys, Spin-up/spin-down models for type Ia supernovae. Astrophys. J. Lett. 738, L1 (2011). 1102.4342. https://doi.org/10.1088/2041-8205/738/1/L1

P.M. Dos Santos, J.R.D. Lepine, Detection of strong $\mathrm{H}_{2} \mathrm{O}$ emission from galaxy NGC4945. Nature 278, 34 (1979). https://doi.org/10.1038/278034a0

A. Dressler, D. Lynden-Bell, D. Burstein, R.L. Davies, S.M. Faber, R. Terlevich, G. Wegner, Spectroscopy and photometry of elliptical galaxies, I: a new distance estimator. Astrophys. J. 313, 42-58 (1987)

P. Du, C. Hu, K.X. Lu, Y.K. Huang, C. Cheng, J. Qiu, Y.R. Li, Y.W. Zhang, X.L. Fan, J.M. Bai, W.H. Bian, Y.F. Yuan, S. Kaspi, L.C. Ho, H. Netzer, J.M. Wang, SEAMBH Collaboration, Supermassive black holes with high accretion rates in active galactic nuclei, IV: $\mathrm{H} \beta$ time lags and implications for super-Eddington accretion. Astrophys. J. 806, 22 (2015). 1504.01844. https://doi.org/10.1088/0004-637X/806/1/22

P. Du, K.X. Lu, Z.X. Zhang, Y.K. Huang, K. Wang, C. Hu, J. Qiu, Y.R. Li, X.L. Fan, X.E. Fang, J.M. Bai, W.H. Bian, Y.F. Yuan, L.C. Ho, J.M. Wang, SEAMBH Collaboration, Supermassive black holes with high accretion rates in active galactic nuclei, $\mathrm{V}$ : a new size-luminosity scaling relation for the broad-line region. Astrophys. J. 825, 126 (2016a). 1604.06218. https://doi.org/10.3847/0004-637X/825/2/126

P. Du, J.M. Wang, C. Hu, L.C. Ho, Y.R. Li, J.M. Bai, The fundamental plane of the broad-line region in active galactic nuclei. Astrophys. J. Lett. 818, L14 (2016b). 1601.01391. https://doi.org/10.3847/ 2041-8205/818/1/L14

R.A. Edelson, T. Alexander, D.M. Crenshaw et al., Multiwavelength observations of short-timescale variability in NGC 4151, IV: analysis of multiwavelength continuum variability. Astrophys. J. 470, 364 (1996). astro-ph/9605082. https://doi.org/10.1086/177872

R. Edelson, J.M. Gelbord, K. Horne et al., Space telescope and optical reverberation mapping project, II: Swift and HST reverberation mapping of the accretion disk of NGC 5548. Astrophys. J. 806, 129 (2015). 1501.05951. https://doi.org/10.1088/0004-637X/806/1/129

A. Einstein, Zur allgemeinen Relativitätstheorie, in Sitzungsberichte der Königlich Preußischen Akademie der Wissenschaften (Berlin) (1915), pp. 778-786

A. Einstein, Näherungsweise Integration der Feldgleichungen der Gravitation, in Sitzungsberichte der Königlich Preußischen Akademie der Wissenschaften (Berlin) (1916), pp. 688-696

A. Einstein, Über Gravitationswellen, in Sitzungsberichte der Königlich Preußischen Akademie der Wissenschaften (Berlin) (1918), pp. 154-167

N. Elias-Rosa, S. Benetti, E. Cappellaro, M. Turatto, P.A. Mazzali, F. Patat, W.P.S. Meikle, M. Stehle, A. Pastorello, G. Pignata, R. Kotak, A. Harutyunyan, G. Altavilla, H. Navasardyan, Y. Qiu, M. Salvo, W. Hillebrandt, Anomalous extinction behaviour towards the type Ia SN 2003cg. Mon. Not. R. Astron. Soc. 369, 1880-1900 (2006). astro-ph/0603316. https://doi.org/10.1111/j.1365-2966.2006.10430.x

N. Elias-Rosa, S. Benetti, M. Turatto, E. Cappellaro, S. Valenti, A.A. Arkharov, J.E. di Beckman, A. Paola, M. Dolci, A.V. Filippenko, R.J. Foley, K. Krisciunas, V.M. Larionov, W. Li, W.P.S. Meikle, A. Pastorello, G. Valentini, W. Hillebrandt, SN 2002cv: a heavily obscured type Ia supernova. Mon. Not. R. Astron. Soc. 384, 107-122 (2008). 0710.4503. https://doi.org/10.1111/j.1365-2966.2007.12638.x

M. Elitzur, Astronomical masers. Annu. Rev. Astron. Astrophys. 30, 75-112 (1992). https://doi.org/10.1146/ annurev.aa.30.090192.000451

M. Elvis, M. Karovska, Quasar parallax: a method for determining direct geometrical distances to quasars. Astrophys. J. Lett. 581, L67-L70 (2002). astro-ph/0211385. https://doi.org/10.1086/346015

I.M.H. Etherington, On the definition of distance in general relativity. Philos. Mag. 15 (1933) 
I.M.H. Etherington, Republication of: LX. On the definition of distance in general relativity. Gen. Relativ. Gravit. 39, 1055-1067 (2007). https://doi.org/10.1007/s 10714-007-0447-X

S.M. Faber, R.E. Jackson, Velocity dispersions and mass-to-light ratios for elliptical galaxies. Astrophys. J. 204, 668-683 (1976). https://doi.org/10.1086/154215

M.M. Fausnaugh, C.S. Kochanek, J.R. Gerke, L.M. Macri, A.G. Riess, K.Z. Stanek, The Cepheid distance to the maser-host galaxy NGC 4258: studying systematics with the Large Binocular Telescope. Mon. Not. R. Astron. Soc. 450, 3597-3619 (2015). 1412.2138. https://doi.org/10.1093/mnras/stv881

M.M. Fausnaugh, K.D. Denney, A.J. Barth et al., Space telescope and optical reverberation mapping project, III: optical continuum emission and broadband time delays in NGC 5548. Astrophys. J. 821, 56 (2016). 1510.05648. https://doi.org/10.3847/0004-637X/821/1/56

A.V. Filippenko, Optical spectra of supernovae. Annu. Rev. Astron. Astrophys. 35, 309-355 (1997a). https:// doi.org/10.1146/annurev.astro.35.1.309

A.V. Filippenko, Optical spectra of supernovae. Annu. Rev. Astron. Astrophys. 35, 309-355 (1997b). https:// doi.org/10.1146/annurev.astro.35.1.309

G. Fiorentino, I. Musella, M. Marconi, Cepheid theoretical models and observations in HST/WFC3 filters: the effect on the Hubble constant $\mathrm{H}_{0}$. Mon. Not. R. Astron. Soc. 434, 2866 (2013). 1306.6276. https:// doi.org/10.1093/mnras/stt1193

E.L. Fitzpatrick, D. Massa, An analysis of the shapes of interstellar extinction curves V: The IR-through-UV curve morphology. Astrophys. J. 663, 320-341 (2007). 0705.0154. https://doi.org/10.1086/518158

G. Folatelli, M.M. Phillips, C.R. Burns, C. Contreras, M. Hamuy, W.L. Freedman, S.E. Persson, M. Stritzinger, N.B. Suntzeff, K. Krisciunas, L. Boldt, S. González, W. Krzeminski, N. Morrell, M. Roth, F. Salgado, B.F. Madore, D. Murphy, P. Wyatt, W. Li, A.V. Filippenko, N. Miller, The Carnegie supernova project: analysis of the first sample of low-redshift type-Ia supernovae. Astron. J. 139, 120-144 (2010). 0910.3317. https://doi.org/10.1088/0004-6256/139/1/120

M. Foley, R. Foley, D. Scolnic, A. Rest, A.G. Riess, S.W. Jha, R. Kirshner, O. Dosovitz Fox, Y.C. Pan, S. Smartt, Calibration and simulation of the foundation supernova survey, in American Astronomical Society Meeting Abstracts, vol. 229 (2017), p. 341.12

R.J. Foley, D. Kasen, Measuring ejecta velocity improves type Ia supernova distances. Astrophys. J. 729, 55 (2011). 1011.4517. https://doi.org/10.1088/0004-637X/729/1/55

R.J. Foley, A.V. Filippenko, C. Aguilera, A.C. Becker, S. Blondin, P. Challis, A. Clocchiatti, R. Covarrubias, T.M. Davis, P.M. Garnavich, S.W. Jha, R.P. Kirshner, K. Krisciunas, B. Leibundgut, W. Li, T. Matheson, A. Miceli, G. Miknaitis, G. Pignata, A. Rest, A.G. Riess, B.P. Schmidt, R.C. Smith, J. Sollerman, J. Spyromilio, C.W. Stubbs, N.B. Suntzeff, J.L. Tonry, W.M. Wood-Vasey, A. Zenteno, Constraining cosmic evolution of type Ia supernovae. Astrophys. J. 684, 68 (2008). 0710.2338. https://doi.org/10.1086/589612

R.J. Foley, T. Matheson, S. Blondin, R. Chornock, J.M. Silverman, P. Challis, A. Clocchiatti, A.V. Filippenko, R.P. Kirshner, B. Leibundgut, J. Sollerman, J. Spyromilio, J.L. Tonry, T.M. Davis, P.M. Garnavich, S.W. Jha, K. Krisciunas, W. Li, G. Pignata, A. Rest, A.G. Riess, B.P. Schmidt, R.C. Smith, C.W. Stubbs, B.E. Tucker, W.M. Wood-Vasey, Spectroscopy of high-redshift supernovae from the essence project: the first four years. Astron. J. 137, 3731-3742 (2009). 0811.4424. https://doi.org/10.1088/ 0004-6256/137/4/3731

R.J. Foley, N.E. Sanders, R.P. Kirshner, Velocity evolution and the intrinsic color of type Ia supernovae. Astrophys. J. 742, 89 (2011). 1107.3555. https://doi.org/10.1088/0004-637X/742/2/89

R.J. Foley, O.D. Fox, C. McCully, M.M. Phillips, D.J. Sand, W. Zheng, P. Challis, A.V. Filippenko, G. Folatelli, W. Hillebrandt, E.Y. Hsiao, S.W. Jha, R.P. Kirshner, M. Kromer, G.H. Marion, M. Nelson, R. Pakmor, G. Pignata, F.K. Röpke, I.R. Seitenzahl, J.M. Silverman, M. Skrutskie, M.D. Stritzinger, Extensive HST ultraviolet spectra and multiwavelength observations of SN 2014J in M82 indicate reddening and circumstellar scattering by typical dust. Mon. Not. R. Astron. Soc. 443, 2887-2906 (2014). 1405.3677. https://doi.org/10.1093/mnras/stu1378

W.L. Freedman, Measuring and modeling the universe (2004)

W.L. Freedman, Cosmology at a crossroads. Nat. Astron. 1, 0121 (2017). https://doi.org/10.1038/ s41550-017-0121

W.L. Freedman, B.F. Madore, The Hubble constant. Annu. Rev. Astron. Astrophys. 48, 673-710 (2010). 1004.1856. https://doi.org/10.1146/annurev-astro-082708-101829

W.L. Freedman, B.F. Madore, B.K. Gibson, L. Ferrarese, D.D. Kelson, S. Sakai, J.R. Mould, R.C. Kennicutt Jr., H.C. Ford, J.A. Graham, J.P. Huchra, S.M.G. Hughes, G.D. Illingworth, L.M. Macri, P.B. Stetson, Final results from the Hubble space telescope key project to measure the Hubble constant. Astrophys. J. 553, 47-72 (2001a). astro-ph/0012376. https://doi.org/10.1086/320638

W.L. Freedman, B.F. Madore, B.K. Gibson, L. Ferrarese, D.D. Kelson, S. Sakai, J.R. Mould, R.C Kennicutt Jr., H.C. Ford, J.A. Graham, J.P. Huchra, S.M.G. Hughes, G.D. Illingworth, L.M. Macri, P.B. Stetson, 
Final results from the Hubble space telescope key project to measure the Hubble constant. Astrophys. J. 553, 47-72 (2001b). astro-ph/0012376. https://doi.org/10.1086/320638

W.L. Freedman, C.R. Burns, M.M. Phillips, P. Wyatt, S.E. Persson, B.F. Madore, C. Contreras, G. Folatelli, E.S. Gonzalez, M. Hamuy, E. Hsiao, D.D. Kelson, N. Morrell, D.C. Murphy, M. Roth, M. Stritzinger, L. Sturch, N.B. Suntzeff, P. Astier, C. Balland, B. Bassett, L. Boldt, R.G. Carlberg, A.J. Conley, J.A. Frieman, P.M. Garnavich, J. Guy, D. Hardin, D.A. Howell, R. Kessler, H. Lampeitl, J. Marriner, R. Pain, K. Perrett, N. Regnault, A.G. Riess, M. Sako, D.P. Schneider, M. Sullivan, M. Wood-Vasey, The Carnegie supernova project: first near-infrared Hubble diagram to $z \sim 0$.7. Astrophys. J. 704, 1036-1058 (2009). 0907.4524. https://doi.org/10.1088/0004-637X/704/2/1036

W.L. Freedman, B.F. Madore, V. Scowcroft, C. Burns, A. Monson, S.E. Persson, M. Seibert, J. Rigby, Carnegie Hubble program: a mid-infrared calibration of the Hubble constant. Astrophys. J. 758(24) (2012). 1208.3281. https://doi.org/10.1088/0004-637X/758/1/24

J.A. Frieman, M.S. Turner, D. Huterer, Dark energy and the accelerating universe. Annu. Rev. Astron. Astrophys. 46, 385-432 (2008). 0803.0982. https://doi.org/10.1146/annurev.astro.46.060407.145243

A. Gal-Yam, P.A. Mazzali, I. Manulis, D. Bishop, Supernova discoveries 2010-2011: statistics and trends. Publ. Astron. Soc. Pac. 125, 749 (2013). 1103.5165. https://doi.org/10.1086/671483

P. Galianni, K. Horne, A test of the failed disc wind scenario for the origin of the broad-line region in active galactic nuclei. Mon. Not. R. Astron. Soc. 435, 3122-3124 (2013). 1306.2835. https://doi.org/ 10.1093/mnras/stt1507

E.E.E. Gall, R. Kotak, B. Leibundgut, S. Taubenberger, W. Hillebrandt, M. Kromer, Applying the expanding photosphere and standardized candle methods to type II-Plateau supernovae at cosmologically significant redshifts. The distance to SN 2013eq. Astron. Astrophys. 592, A129 (2016). 1603.04730. https://doi.org/10.1051/0004-6361/201628333

J. Gao, B.W. Jiang, A. Li, J. Li, X. Wang, Physical dust models for the extinction toward supernova 2014J in M82. Astrophys. J. Lett. 807, L26 (2015). 1507.00417. https://doi.org/10.1088/2041-8205/807/2/L26

D. García-Senz, C. Badenes, N. Serichol, Is there a hidden hole in type Ia supernova remnants? Astrophys. J. 745, 75 (2012). 1110.4267. https://doi.org/10.1088/0004-637X/745/1/75

E. Gardner, C. Done, The UV/optical lags in NGC 5548: it's not disc reprocessing and the soft X-ray excess is part of the solution. 1603.09564 (2016)

C.M. Gaskell, L.S. Sparke, Line variations in quasars and Seyfert galaxies. Astrophys. J. 305, 175-186 (1986). https://doi.org/10.1086/164238

M. Gierliński, M. Nikołajuk, B. Czerny, High-frequency X-ray variability as a mass estimator of stellar and supermassive black holes. Mon. Not. R. Astron. Soc. 383, 741-749 (2008). 0710.1566. https://doi.org/10.1111/j.1365-2966.2007.12584.x

M. Gilfanov, Á. Bogdán, An upper limit on the contribution of accreting white dwarfs to the type Ia supernova rate. Nature 463, 924-925 (2010). 1002.3359. https://doi.org/10.1038/nature08685

A. Goobar, Low $R_{V}$ from circumstellar dust around supernovae. Astrophys. J. Lett. 686, L103 (2008). 0809.1094. https://doi.org/10.1086/593060

A. Goobar, B. Leibundgut, Supernova cosmology: legacy and future. Annu. Rev. Nucl. Part. Sci. 61, 251-279 (2011). 1102.1431. https://doi.org/10.1146/annurev-nucl-102010-130434

M.L. Graham, S. Valenti, B.J. Fulton, L.M. Weiss, K.J. Shen, P.L. Kelly, W. Zheng, A.V. Filippenko, G.W. Marcy, D.A. Howell, J. Burt, E.J. Rivera, Time-varying potassium in high-resolution spectra of the type Ia supernova 2014j. Astrophys. J. 801, 136 (2015). 1412.0653. https://doi.org/10.1088/ 0004-637X/801/2/136

E.K. Grassberg, V.S. Imshennik, D.K. Nadyozhin, On the theory of the light curves of supernovate. Astrophys. Space Sci. 10, 28-51 (1971). https://doi.org/10.1007/BF00654604

D. Grupe, S. Komossa, K.M. Leighly, K.L. Page, The simultaneous optical-to-X-ray spectral energy distribution of soft X-ray selected active galactic nuclei observed by Swift. Astrophys. J. Suppl. 187, 64-106 (2010). 1001.3140. https://doi.org/10.1088/0067-0049/187/1/64

J. Guillochon, J. Parrent, R. Margutti, An open catalog for supernova data. 1605.01054 (2016)

J. Guy, P. Astier, S. Nobili, N. Regnault, R. Pain, SALT: a spectral adaptive light curve template for type Ia supernovae. Astron. Astrophys. 443, 781-791 (2005). astro-ph/0506583. https://doi.org/10.1051/ 0004-6361:20053025

J. Guy, P. Astier, S. Baumont, D. Hardin, R. Pain, N. Regnault, S. Basa, R.G. Carlberg, A. Conley, S. Fabbro, D. Fouchez, I.M. Hook, D.A. Howell, K. Perrett, C.J. Pritchet, J. Rich, M. Sullivan, P. Antilogus, E. Aubourg, G. Bazin, J. Bronder, M. Filiol, N. Palanque-Delabrouille, P. Ripoche, V. Ruhlmann-Kleider, SALT2: using distant supernovae to improve the use of type Ia supernovae as distance indicators. Astron. Astrophys. 466, 11-21 (2007). astro-ph/0701828. https://doi.org/10.1051/0004-6361:20066930

M. Haas, R. Chini, M. Ramolla, F. Pozo Nuñez, C. Westhues, R. Watermann, V. Hoffmeister, M. Murphy, Photometric AGN reverberation mapping-an efficient tool for BLR sizes, black hole masses, 
and host-subtracted AGN luminosities. Astron. Astrophys. 535, A73 (2011). 1109.1848. https://doi.org/ 10.1051/0004-6361/201117325

I. Hachisu, M. Kato, A theoretical light-curve model for the recurrent nova V394 coronae Australis. Astrophys. J. 540, 447-451 (2000). astro-ph/0003471. https://doi.org/10.1086/309338

I. Hachisu, M. Kato, K. Nomoto, A new model for progenitor systems of type IA supernovae. Astrophys. J. Lett. 470, L97 (1996). https://doi.org/10.1086/310303

I. Hachisu, M. Kato, K. Nomoto, Supersoft X-ray phase of single degenerate type Ia supernova progenitors in early-type galaxies. Astrophys. J. Lett. 724, L212-L216 (2010). 1010.5860. https://doi.org/ $10.1088 / 2041-8205 / 724 / 2 /$ L2 212

M. Hamuy, The standard candle method for type II supernovae and the Hubble constant. astro-ph/0309122 (2003)

M. Hamuy, The latest version of the standardized candle method for type II supernovae. Measuring and modeling the universe. astro-ph/0301281 (2004)

M. Hamuy, P.A. Pinto, Type II supernovae as standardized candles. Astrophys. J. Lett. 566, L63-L65 (2002). astro-ph/0201279. https://doi.org/10.1086/339676

M. Hamuy, M.M. Phillips, J. Maza, N.B. Suntzeff, R.A. Schommer, R. Aviles, A Hubble diagram of distant type IA supernovae. Astron. J. 109, 1-13 (1995). https://doi.org/10.1086/117251

M. Hamuy, M.M. Phillips, N.B. Suntzeff, R.A. Schommer, J. Maza, R. Aviles, The absolute luminosities of the Calan/Tololo type IA supernovae. Astron. J. 112, 2391 (1996). astro-ph/9609059. https://doi.org/ 10.1086/118190

M. Hamuy, G. Folatelli, N.I. Morrell, M.M. Phillips, N.B. Suntzeff, S.E. Persson, M. Roth, S. Gonzalez, W. Krzeminski, C. Contreras, W.L. Freedman, D.C. Murphy, B.F. Madore, P. Wyatt, J. Maza, A.V. Filippenko, W. Li, P.A. Pinto, The Carnegie supernova project: the low-redshift survey. Publ. Astron. Soc. Pac. 118, 2-20 (2006). astro-ph/0512039. https://doi.org/10.1086/500228

A. Heger, C.L. Fryer, S.E. Woosley, N. Langer, D.H. Hartmann, How massive single stars end their life. Astrophys. J. 591, 288-300 (2003). astro-ph/0212469. https://doi.org/10.1086/375341

J.R. Herrnstein, J.M. Moran, L.J. Greenhill, A.S. Trotter, The geometry of and mass accretion rate through the maser accretion disk in NGC 4258. Astrophys. J. 629, 719-738 (2005). astro-ph/0504405. https:// doi.org/10.1086/431421

M. Hicken, W.M. Wood-Vasey, S. Blondin, P. Challis, S. Jha, P.L. Kelly, A. Rest, R.P. Kirshner, Improved dark energy constraints from 100 new CfA supernova type Ia light curves. Astrophys. J. 700, 10971140 (2009). 0901.4804. https://doi.org/10.1088/0004-637X/700/2/1097

S. Hilbert, J.R. Gair, L.J. King, Reducing distance errors for standard candles and standard sirens with weak-lensing shear and flexion maps. Mon. Not. R. Astron. Soc. 412, 1023-1037 (2011). 1007.2468. https://doi.org/10.1111/j.1365-2966.2010.17963.x

J. Hjorth, J.S. Bloom, in The Gamma-Ray Burst-Supernova Connection (2012), pp. 169-190

L.C. Ho, Z.Y. Li, A.J. Barth, M.S. Seigar, C.Y. Peng, The Carnegie-Irvine galaxy survey, I: overview and atlas of optical images. Astrophys. J. Suppl. 197, 21 (2011). 1111.4605. https://doi.org/10.1088/0067-0049/ $197 / 2 / 21$

R.F.L. Holanda, K.N.N.O. Barros, Searching for cosmological signatures of the Einstein equivalence principle breaking. Phys. Rev. D 94(2), 023524 (2016). 1606.07923. https://doi.org/10.1103/PhysRevD. 94.023524

R.F.L. Holanda, J.A.S. Lima, M.B. Ribeiro, Testing the distance-duality relation with galaxy clusters and type Ia supernovae. Astrophys. J. Lett. 722, L233-L237 (2010). 1005.4458. https://doi.org/10.1088/ 2041-8205/722/2/L233

R.F.L. Holanda, J.V. Cunha, L. Marassi, J.A.S. Lima, Constraining $\mathrm{H}_{0}$ in general dark energy models from Sunyaev-Zeldovich/X-ray technique and complementary probes. J. Cosmol. Astropart. Phys. 2, 035 (2012a). 1006.4200. https://doi.org/10.1088/1475-7516/2012/02/035

R.F.L. Holanda, J.A.S. Lima, M.B. Ribeiro, Probing the cosmic distance-duality relation with the SunyaevZel'dovich effect, X-ray observations and supernovae Ia. Astron. Astrophys. 538, A131 (2012b). 1104.3753. https://doi.org/10.1051/0004-6361/201118343

R.F.L. Holanda, J.S. Alcaniz, J.C. Carvalho, Cosmography with the Sunyaev-Zeldovich effect and X-ray data. J. Cosmol. Astropart. Phys. 6, 033 (2013). 1303.3307. https://doi.org/10.1088/1475-7516/2013/06/033

R.F.L. Holanda, V.C. Busti, L.R. Colaço, J.S. Alcaniz, S.J. Landau, Galaxy clusters, type Ia supernovae and the fine structure constant. J. Cosmol. Astropart. Phys. 8, 055 (2016). 1605.02578. https://doi.org/ $10.1088 / 1475-7516 / 2016 / 08 / 055$

T.W.S. Holoien, J.S. Brown, K.Z. Stanek, C.S. Kochanek, B.J. Shappee, J.L. Prieto, S. Dong, J. Brimacombe, D.W. Bishop, U. Basu, J.F. Beacom, D. Bersier, P. Chen, A.B. Danilet, E. Falco, D. GodoyRivera, N. Goss, G. Pojmanski, G.V. Simonian, D.M. Skowron, T.A. Thompson, P.R. Woźniak, C.G. Ávila, G. Bock, J.L.G. Carballo, E. Conseil, C. Contreras, I. Cruz, J.M.F. Andújar, Z. Guo, E.Y. Hsiao, S. Kiyota, R.A. Koff, G. Krannich, B.F. Madore, P. Marples, G. Masi, N. Morrell, L.A.G. 
Monard, J.C. Munoz-Mateos, B. Nicholls, J. Nicolas, R.M. Wagner, W.S. Wiethoff, The ASAS-SN bright supernova catalogue, II: 2015. Mon. Not. R. Astron. Soc. 467, 1098-1111 (2017a). 1610.03061. https://doi.org/10.1093/mnras/stx057

T.W.S. Holoien, J.S. Brown, K.Z. Stanek, C.S. Kochanek, B.J. Shappee, J.L. Prieto, S. Dong, J. Brimacombe, D.W. Bishop, S. Bose, J.F. Beacom, D. Bersier, P. Chen, L. Chomiuk, E. Falco, D. Godoy-Rivera, N. Morrell, G. Pojmanski, J.V. Shields, J. Strader, M.D. Stritzinger, T.A. Thompson, P.R. Woźniak, G. Bock, P. Cacella, E. Conseil, I. Cruz, J.M. Fernandez, S. Kiyota, R.A. Koff, G. Krannich, P. Marples, G. Masi, L.A.G. Monard, B. Nicholls, J. Nicolas, R.S. Post, G. Stone, W.S. Wiethoff, The ASAS-SN bright supernova catalog, III: 2016. 1704.02320 (2017b)

T.W.S. Holoien, P.J. Marshall, R.H. Wechsler, EmpiriciSN: re-sampling observed supernova/host galaxy populations using an XD Gaussian mixture model. Astron. J. 153, 249 (2017c). 1611.00363. https://doi.org/ 10.3847/1538-3881/aa68a1

T.W.S. Holoien, K.Z. Stanek, C.S. Kochanek, B.J. Shappee, J.L. Prieto, J. Brimacombe, D. Bersier, D.W. Bishop, S. Dong, J.S. Brown, A.B. Danilet, G.V. Simonian, U. Basu, J.F. Beacom, E. Falco, G. Pojmanski, D.M. Skowron, P.R. Woźniak, C.G. Ávila, E. Conseil, C. Contreras, I. Cruz, J.M. Fernández, R.A. Koff, Z. Guo, G.J. Herczeg, J. Hissong, E.Y. Hsiao, J. Jose, S. Kiyota, F. Long, L.A.G. Monard, B. Nicholls, J. Nicolas, W.S. Wiethoff, The ASAS-SN bright supernova catalogue, I: 2013-2014. Mon. Not. R. Astron. Soc. 464, 2672-2686 (2017d). 1604.00396. https://doi.org/10.1093/mnras/stw2273

D.E. Holz, S.A. Hughes, Using gravitational-wave standard sirens. Astrophys. J. 629, 15-22 (2005). astro-ph/ 0504616. https://doi.org/10.1086/431341

S.F. Hönig, D. Watson, M. Kishimoto, J. Hjorth, A dust-parallax distance of 19 megaparsecs to the supermassive black hole in NGC 4151. Nature 515, 528-530 (2014). 1411.7032. https://doi.org/10.1038/ nature 13914

I.M. Hook, Supernovae and cosmology with future European facilities. Philos. Trans. R. Soc. Lond. Ser. A 371, 20120 (2013). 1211.6586. https://doi.org/10.1098/rsta.2012.0282

A.M. Hopkins, J.F. Beacom, On the normalization of the cosmic star formation history. Astrophys. J. 651, 142-154 (2006). astro-ph/0601463. https://doi.org/10.1086/506610

G. Hosseinzadeh, D.A. Howell, D. Sand, S. Valenti, L. Tartaglia, C. McCully, K.A. Bostroem, S. Wyatt, I. Arcavi, FLOYDS classification of DLT17u/AT 2017cbv as a very young type Ia supernova. Astron. Telegr. 10164, 164 (2017)

D.A. Howell, M. Sullivan, K. Perrett, T.J. Bronder, I.M. Hook, P. Astier, E. Aubourg, D. Balam, S. Basa, R.G. Carlberg, S. Fabbro, D. Fouchez, J. Guy, H. Lafoux, J.D. Neill, R. Pain, N. Palanque-Delabrouille, C.J. Pritchet, N. Regnault, J. Rich, R. Taillet, R. Knop, R.G. McMahon, S. Perlmutter, N.A. Walton, Gemini spectroscopy of supernovae from the supernova legacy survey: improving high-redshift supernova selection and classification. Astrophys. J. 6(34), 1190-1201 (2005). astro-ph/0509195. https://doi.org/10.1086/497119

F. Hoyle, W.A. Fowler, Nucleosynthesis in supernovae. Astrophys. J. 132, 565 (1960). https://doi.org/ $10.1086 / 146963$

K. Hryniewicz, B. Czerny, W. Pych, A. Udalski, M. Krupa, A. Świętoń, J. Kaluzny, SALT long-slit spectroscopy of LBQS 2113-4538: variability of the Mg II and Fe II component. Astron. Astrophys. 562, A34 (2014). 1308.3980. https://doi.org/10.1051/0004-6361/201322487

E.P. Hubble, A spiral nebula as a stellar system, Messier 31. Astrophys. J. 69 (1929). https://doi.org/10.1086/ 143167

E.M.L. Humphreys, M.J. Reid, J.M. Moran, L.J. Greenhill, A.L. Argon, Toward a new geometric distance to the active galaxy NGC 4258, III: final results and the Hubble constant. Astrophys. J. 775, 13 (2013). 1307.6031. https://doi.org/10.1088/0004-637X/775/1/13

K. Ichikawa, C. Ricci, Y. Ueda, K. Matsuoka, Y. Toba, T. Kawamuro, B. Trakhtenbrot, M.J. Koss, The complete infrared view of active galactic nuclei from the 70 month Swift/BAT catalog. Astrophys. J. 835, 74 (2017). 1611.09858. https://doi.org/10.3847/1538-4357/835/1/74

D. Ilić, P. LČ , A.I. Shapovalova, A.N. Burenkov, V.H. Chavushyan, A. Kovačević, Line shape variability in a sample of AGN with broad lines. J. Astrophys. Astron. 36, 433-445 (2015). 1510.02162. https://doi.org/ 10.1007/s12036-015-9360-y

N. Itoh, Y. Kohyama, S. Nozawa, Relativistic corrections to the Sunyaev-Zeldovich effect for clusters of galaxies. Astrophys. J. 502, 7-15 (1998). astro-ph/9712289. https://doi.org/10.1086/305876

N. Ivanova, S. Justham, X. Chen, O. De Marco, C.L. Fryer, E. Gaburov, H. Ge, E. Glebbeek, Z. Han, X.D. Li, G. Lu, T. Marsh, P. Podsiadlowski, A. Potter, N. Soker, R. Taam, T.M. Tauris, E.P.J. van den Heuvel, R.F. Webbink, Common envelope evolution: where we stand and how we can move forward. Astron. Astrophys. Rev. 21, 59 (2013). 1209.4302. https://doi.org/10.1007/s00159-013-0059-2

D. Jack, M. Mittag, K.P. Schröder, J.H.M.M. Schmitt, A. Hempelmann, J.N. González-Pérez, M.A. Trinidad, J.M. Cabrera Sixto, G. Rauw, Time series of high-resolution spectra of SN 2014J ob- 
served with the TIGRE telescope. Mon. Not. R. Astron. Soc. 451, 4104-4113 (2015). 1506.00938. https://doi.org/10.1093/mnras/stv1238

I.S. Jang, M.G. Lee, The tip of the red giant branch distance to the perfect spiral galaxy M74 hosting three core-collapse supernovae. Astrophys. J. 792, 52 (2014). 1407.2246. https://doi.org/10.1088/0004-637X/ $792 / 1 / 52$

J.B. Jensen, J.P. Blakeslee, Z. Gibson, H.C. Lee, M. Cantiello, G. Raimondo, N. Boyer, H. Cho, Measuring infrared surface brightness fluctuation distances with HST WFC3: calibration and advice. Astrophys. J. 808, 91 (2015). 1505.00400. https://doi.org/10.1088/0004-637X/808/1/91

S. Jha, R.P. Kirshner, P. Challis, P.M. Garnavich, T. Matheson, A.M. Soderberg, G.J.M. Graves, M. Hicken, J.F. Alves, H.G. Arce, Z. Balog, P. Barmby, E.J. Barton, P. Berlind, A.E. Bragg, C. Briceño, W.R. Brown, J.H. Buckley, N. Caldwell, M.L. Calkins, B.J. Carter, K.D. Concannon, R.H. Donnelly, K.A. Eriksen, D.G. Fabricant, E.E. Falco, F. Fiore, M.R. Garcia, M. Gómez, N.A. Grogin, T. Groner, P.J. Groot, K.E. Haisch Jr., L. Hartmann, C.W. Hergenrother, M.J. Holman, J.P. Huchra, R. Jayawardhana, D. Jerius, S.J. Kannappan, D.W. Kim, J.T. Kleyna, C.S. Kochanek, D.M. Koranyi, M. Krockenberger, C.J. Lada, K.L. Luhman, J.X. Luu, L.M. Macri, J.A. Mader, A. Mahdavi, M. Marengo, B.G. Marsden, B.A. McLeod, B.R. McNamara, S.T. Megeath, D. Moraru, A.E. Mossman, A.A. Muench, J.A. Muñoz, J. Muzerolle, O. Naranjo, K. Nelson-Patel, M.A. Pahre, B.M. Patten, J. Peters, W. Peters, J.C. Raymond, K. Rines, R.E. Schild, G.J. Sobczak, T.B. Spahr, J.R. Stauffer, R.P. Stefanik, A.H. Szentgyorgyi, E.V. Tollestrup, P. Väisänen, A. Vikhlinin, Z. Wang, S.P. Willner, S.J. Wolk, J.M. Zajac, P. Zhao, K.Z. Stanek, UBVRI light curves of 44 type Ia supernovae. Astron. J. 131, 527-554 (2006). astro-ph/0509234. https://doi.org/10.1086/497989

S. Jha, A.G. Riess, R.P. Kirshner, Improved distances to type Ia supernovae with multicolor light-curve shapes: MLCS2k2. Astrophys. J. 659, 122-148 (2007). astro-ph/0612666. https://doi.org/10.1086/ 512054

D.O. Jones, S.A. Rodney, A.G. Riess, B. Mobasher, T. Dahlen, C. McCully, T.F. Frederiksen, S. Casertano, J. Hjorth, C.R. Keeton, A. Koekemoer, L.G. Strolger, T.G. Wiklind, P. Challis, O. Graur, B. Hayden, B. Patel, B.J. Weiner, A.V. Filippenko, P. Garnavich, S.W. Jha, R.P. Kirshner, H.C. Ferguson, N.A. Grogin, D. Kocevski, The discovery of the most distant known type Ia supernova at redshift 1.914. Astrophys. J. 768, 166 (2013). 1304.0768. https://doi.org/10.1088/0004-637X/768/2/166

D.O. Jones, A.G. Riess, D.M. Scolnic, Reconsidering the effects of local star formation on type Ia supernova cosmology. Astrophys. J. 812, 31 (2015). 1506.02637. https://doi.org/10.1088/0004-637X/812/1/31

D.W. Just, W.N. Brandt, O. Shemmer, A.T. Steffen, D.P. Schneider, G. Chartas, G.P. Garmire, The X-ray properties of the most luminous quasars from the sloan digital sky survey. Astrophys. J. 665, 10041022 (2007). 0705.3059. https://doi.org/10.1086/519990

S. Justham, Single-degenerate type Ia supernovae without hydrogen contamination. Astrophys. J. Lett. 730, L34 (2011). 1102.4913. https://doi.org/10.1088/2041-8205/730/2/L34

D. Kasen, S.E. Woosley, On the origin of the type Ia supernova width-luminosity relation. Astrophys. J. 656, 661-665 (2007). astro-ph/0609540. https://doi.org/10.1086/510375

D. Kasen, S.E. Woosley, Type II supernovae: model light curves and standard candle relationships. Astrophys. J. 703, 2205-2216 (2009). 0910.1590. https://doi.org/10.1088/0004-637X/703/2/2205

D. Kasen, F.K. Röpke, S.E. Woosley, The diversity of type Ia supernovae from broken symmetries. Nature 460, 869-872 (2009). 0907.0708. https://doi.org/10.1038/nature08256

S. Kaspi, P.S. Smith, H. Netzer, D. Maoz, B.T. Jannuzi, U. Giveon, Reverberation measurements for 17 quasars and the size-mass-luminosity relations in active galactic nuclei. Astrophys. J. 533, 631-649 (2000). astro-ph/9911476. https://doi.org/10.1086/308704

S. Kaspi, W.N. Brandt, D. Maoz, H. Netzer, D.P. Schneider, O. Shemmer, Reverberation mapping of highluminosity quasars: first results. Astrophys. J. 659, 997-1007 (2007). astro-ph/0612722. https://doi.org/ $10.1086 / 512094$

K.S. Kawabata, H. Akitaya, M. Yamanaka, R. Itoh, K. Maeda, Y. Moritani, T. Ui, M. Kawabata, K. Mori, D. Nogami, K. Nomoto, N. Suzuki, K. Takaki, M. Tanaka, I. Ueno, S. Chiyonobu, T. Harao, R. Matsui, H. Miyamoto, O. Nagae, A. Nakashima, H. Nakaya, Y. Ohashi, T. Ohsugi, T. Komatsu, K. Sakimoto, M. Sasada, H. Sato, H. Tanaka, T. Urano, T. Yamashita, M. Yoshida, A. Arai, N. Ebisuda, Y. Fukazawa, A. Fukui, O. Hashimoto, S. Honda, H. Izumiura, Y. Kanda, K. Kawaguchi, N. Kawai, D. Kuroda, K. Masumoto, K. Matsumoto, T. Nakaoka, K. Takata, M. Uemura, K. Yanagisawa, Optical and near-infrared polarimetry of highly reddened type Ia supernova 2014J: peculiar properties of dust in M82. Astrophys. J. Lett. 795, L4 (2014). 1407.0452. https://doi.org/10.1088/2041-8205/795/1/L4

W.E. Kerzendorf, B.P. Schmidt, M. Asplund, K. Nomoto, P. Podsiadlowski, A. Frebel, R.A. Fesen, D. Yong, Subaru high-resolution spectroscopy of star G in the Tycho supernova remnant. Astrophys. J. 701, 16651672 (2009). 0906.0982. https://doi.org/10.1088/0004-637X/701/2/1665 
A.L. King, T.M. Davis, K.D. Denney, M. Vestergaard, D. Watson, High-redshift standard candles: predicted cosmological constraints. Mon. Not. R. Astron. Soc. 441, 3454-3476 (2014). 1311.2356. https://doi.org/ 10.1093/mnras/stu793

A.L. King, P. Martini, T.M. Davis, K.D. Denney, C.S. Kochanek, B.M. Peterson, A. Skielboe, M. Vestergaard, E. Huff, D. Watson, M. Banerji, R. McMahon, R. Sharp, C. Lidman, Simulations of the OzDES AGN reverberation mapping project. Mon. Not. R. Astron. Soc. 453, 1701-1726 (2015). 1504.03031. https://doi.org/10.1093/mnras/stv1718

R.P. Kirshner, J. Kwan, Distances to extragalactic supernovae. Astrophys. J. 193, 27-36 (1974). https:// doi.org/10.1086/153123

M. Kishimoto, S.F. Hönig, R. Antonucci, F. Millour, K.R.W. Tristram, G. Weigelt, Mapping the radial structure of AGN tori. Astron. Astrophys. 536, A78 (2011). 1110.4290. https://doi.org/10.1051/ 0004-6361/201117367

Y. Kobayashi, Y. Yoshii, B.A. Peterson, S. Miyazaki, T. Aoki, T. Minezaki, K. Kawara, K. Enya, N. Okada, M. Suganuma, B. Greene, M. O'Brien, L.K. Randall, MAGNUM (multicolor active galactic nuclei monitoring) project, in Advanced Technology Optical/IR Telescopes VI, ed. by L.M. Stepp. Proc. SPIE, vol. 3352 (1998), pp. 120-128. https://doi.org/10.1117/12.319247

K.T. Korista, M.R. Goad, The variable diffuse continuum emission of broad-line clouds. Astrophys. J. 553, 695-708 (2001). astro-ph/0101117. https://doi.org/10.1086/320964

S. Koshida, T. Minezaki, Y. Yoshii, Y. Kobayashi, Y. Sakata, S. Sugawara, K. Enya, M. Suganuma, H. Tomita, T. Aoki, B.A. Peterson, Reverberation measurements of the inner radius of the dust torus in 17 Seyfert galaxies. Astrophys. J. 788, 159 (2014). 1406.2078. https://doi.org/10.1088/0004-637X/788/2/159

S. Koshida, Y. Yoshii, Y. Kobayashi, T. Minezaki, K. Enya, M. Suganuma, H. Tomita, T. Aoki, B.A. Peterson, Calibration of AGN reverberation distance measurements. Astrophys. J. Lett. 842, L13 (2017). 1705.09757. https://doi.org/10.3847/2041-8213/aa7553

K. Krisciunas, J.L. Prieto, P.M. Garnavich, J.L.G. Riley, A. Rest, C. Stubbs, R. McMillan, Photometry of the type Ia supernovae 1999cc, 1999cl, and 2000cf. Astron. J. 131, 1639-1647 (2006). astro-ph/0511162. https://doi.org/10.1086/499523

K. Krisciunas, P.M. Garnavich, V. Stanishev, N.B. Suntzeff, J.L. Prieto, J. Espinoza, D. Gonzalez, M.E. Salvo, N. Elias de la Rosa, S.J. Smartt, J.R. Maund, R.P. Kudritzki, The type Ia supernova 2004S, a clone of SN 2001el, and the optimal photometric bands for extinction estimation. Astron. J. 133, 58-72 (2007). astro-ph/0609268. https://doi.org/10.1086/509126

A. Królak, B.F. Schutz, Coalescing binaries_probe of the universe. Gen. Relativ. Gravit. 19, 1163-1171 (1987). https://doi.org/10.1007/BF00759095

F. La Franca, S. Bianchi, G. Ponti, E. Branchini, G. Matt, A new cosmological distance measure using active galactic nucleus X-ray variability. Astrophys. J. Lett. 787, L12 (2014). 1404.2607. https://doi.org/ 10.1088/2041-8205/787/1/L12

R.N. Lang, S.A. Hughes, Localizing coalescing massive black hole binaries with gravitational waves. Astrophys. J. 677, 1184-1200 (2008). arXiv:0710.3795. https://doi.org/10.1086/528953

D.C. Leonard, Constraining the type Ia supernova progenitor: the search for hydrogen in nebular spectra. Astrophys. J. 670, 1275-1282 (2007). 0710.3166. https://doi.org/10.1086/522367

D.C. Leonard, S.M. Kanbur, C.C. Ngeow, N.R. Tanvir, The Cepheid distance to NGC 1637: a direct test of the expanding photosphere method distance to SN 1999em. Astrophys. J. 594, 247-278 (2003). astro-ph/0305259. https://doi.org/10.1086/376831

K. Lepo, M. van Kerkwijk, A search for rapidly accreting white dwarfs in the Small Magellanic Cloud. Astrophys. J. 771, 13 (2013). 1301.1368. https://doi.org/10.1088/0004-637X/771/1/13

P. Lesaffre, Z. Han, C.A. Tout, P. Podsiadlowski, R.G. Martin, The C flash and the ignition conditions of type Ia supernovae. Mon. Not. R. Astron. Soc. 368, 187-195 (2006). astro-ph/0601443. https://doi.org/ 10.1111/j.1365-2966.2006.10068.x

W. Li, A.V. Filippenko, R.R. Treffers, A.G. Riess, J. Hu, Y. Qiu, A high intrinsic peculiarity rate among type IA supernovae. Astrophys. J. 546, 734-743 (2001). astro-ph/0006292. https://doi.org/10.1086/318299

W. Li, J.S. Bloom, P. Podsiadlowski, A.A. Miller, S.B. Cenko, S.W. Jha, M. Sullivan, D.A. Howell, P.E. Nugent, N.R. Butler, E.O. Ofek, M.M. Kasliwal, J.W. Richards, A. Stockton, H.Y. Shih, L. Bildsten, M.M. Shara, J. Bibby, A.V. Filippenko, M. Ganeshalingam, J.M. Silverman, S.R. Kulkarni, N.M. Law, D. Poznanski, R.M. Quimby, C. McCully, B. Patel, K. Maguire, K.J. Shen, Exclusion of a luminous red giant as a companion star to the progenitor of supernova SN 2011fe. Nature 480, 348-350 (2011a). 1109.1593. https://doi.org/10.1038/nature10646

Z. Li, P. Wu, H. Yu, Cosmological-model-independent tests for the distance-duality relation from galaxy clusters and type Ia supernova. Astrophys. J. Lett. 729, L14 (2011b). 1101.5255. https://doi.org/ $10.1088 / 2041-8205 / 729 / 1 /$ L14

P. Lira, I. Botti, H. Netzer, S. Kaspi, Reverberation mapping of the most luminous quasars at $z \sim 2-3$, in Active Galactic Nuclei: What's in a Name? (2016), p. 10. https://doi.org/10.5281/zenodo.60361 
I.I. Litvinova, D.K. Nadezhin, Hydrodynamical models of type II supernovae. Astrophys. Space Sci. 89, 89-113 (1983). https://doi.org/10.1007/BF01008387

K.Y. Lo, Mega-masers and galaxies. Annu. Rev. Astron. Astrophys. 43, 625-676 (2005). https://doi.org/ 10.1146/annurev.astro.41.011802.094927

LSST Science Collaboration, P.A. Abell, J. Allison, S.F. Anderson, J.R. Andrew, J.R.P. Angel, L. Armus, D. Arnett, S.J. Asztalos, T.S. Axelrod et al., LSST science book, version 2.0. 0912.0201 (2009)

E. Lusso, G. Risaliti, The tight relation between X-ray and ultraviolet luminosity of quasars. Astrophys. J. 819, 154 (2016). 1602.01090. https://doi.org/10.3847/0004-637X/819/2/154

E. Lusso, A. Comastri, C. Vignali, G. Zamorani, M. Brusa, R. Gilli, K. Iwasawa, M. Salvato, F. Civano, M. Elvis, A. Merloni, A. Bongiorno, J.R. Trump, A.M. Koekemoer, E. Schinnerer, E. Le Floc'h, N. Cappelluti, K. Jahnke, M. Sargent, J. Silverman, V. Mainieri, F. Fiore, M. Bolzonella, O. Le Fèvre, B. Garilli, A. Iovino, J.P. Kneib, F. Lamareille, S. Lilly, M. Mignoli, M. Scodeggio, D. Vergani, The X-ray to optical-UV luminosity ratio of X-ray selected type 1 AGN in XMM-COSMOS. Astron. Astrophys. 512, A34 (2010). 0912.4166. https://doi.org/10.1051/0004-6361/200913298

C.P. Ma, J.E. Greene, N. McConnell, R. Janish, J.P. Blakeslee, J. Thomas, J.D. Murphy, The MASSIVE survey, I: a volume-limited integral-field spectroscopic study of the most massive early-type galaxies within 108 Mpc. Astrophys. J. 795, 158 (2014). 1407.1054. https://doi.org/10.1088/0004-637X/795/2/158

C.L. MacLeod, C.J. Hogan, Precision of Hubble constant derived using black hole binary absolute distances and statistical redshift information. Phys. Rev. D 77(4), 043512 (2008). 0712.0618. https:// doi.org/10.1103/PhysRevD.77.043512

K. Maeda, T. Nozawa, T. Nagao, K. Motohara, Constraining the amount of circumstellar matter and dust around type Ia supernovae through near-infrared echoes. Mon. Not. R. Astron. Soc. 452, 3281-3292 (2015). 1411.3778. https://doi.org/10.1093/mnras/stv1498

K. Maeda, A. Tajitsu, K.S. Kawabata, R.J. Foley, S. Honda, Y. Moritani, M. Tanaka, O. Hashimoto, M. Ishigaki, J.D. Simon, M.M. Phillips, M. Yamanaka, D. Nogami, A. Arai, W. Aoki, K. Nomoto, D. Milisavljevic, P.A. Mazzali, A.M. Soderberg, M. Schramm, B. Sato, H. Harakawa, N. Morrell, N. Arimoto, Sodium absorption systems toward SN Ia 2014J originate on interstellar scales. Astrophys. J.816:57 (2016). 1511.05668. https://doi.org/10.3847/0004-637X/816/2/57

K. Maguire, R. Kotak, S.J. Smartt, A. Pastorello, M. Hamuy, F. Bufano, Type II-P supernovae as standardized candles: improvements using near-infrared data. Mon. Not. R. Astron. Soc. 403, L11-L15 (2010). 0912.3107. https://doi.org/10.1111/j.1745-3933.2009.00804.x

K.S. Mandel, G. Narayan, R.P. Kirshner, Type Ia supernova light curve inference: hierarchical models in the optical and near-infrared. Astrophys. J. 731, 120 (2011). 1011.5910. https://doi.org/10.1088/ 0004-637X/731/2/120

K.S. Mandel, D. Scolnic, H. Shariff, R.J. Foley, R.P. Kirshner, The type Ia supernova color-magnitude relation and host galaxy dust: a simple hierarchical Bayesian model. 1609.04470 (2016)

D. Maoz, O. Graur, Star formation, supernovae, iron, and $\alpha$ : consistent cosmic and galactic histories. 1703.04540 (2017)

D. Maoz, F. Mannucci, Type-Ia supernova rates and the progenitor problem: a review. Publ. Astron. Soc. Aust. 29, 447-465 (2012). 1111.4492. https://doi.org/10.1071/AS11052

D. Maoz, F. Mannucci, T.D. Brandt, The delay-time distribution of type Ia supernovae from Sloan II. Mon. Not. R. Astron. Soc. 426, 3282-3294 (2012). 1206.0465. https://doi.org/10.1111/j.1365-2966. 2012.21871.X

D. Maoz, F. Mannucci, G. Nelemans, Observational clues to the progenitors of type Ia supernovae. Annu. Rev. Astron. Astrophys. 52, 107-170 (2014). 1312.0628. https://doi.org/10.1146/ annurev-astro-082812-141031

P. Massey, E. Waterhouse, K. DeGioia-Eastwood, The progenitor masses of Wolf-Rayet stars and luminous blue variables determined from cluster turnoffs, I: results from 19 OB associations in the Magellanic Clouds. Astron. J. 119, 2214-2241 (2000). astro-ph/0002233. https://doi.org/10.1086/301345

P. Massey, K. DeGioia-Eastwood, E. Waterhouse, The progenitor masses of Wolf-Rayet stars and luminous blue variables determined from cluster turnoffs, II: results from 12 galactic clusters and OB associations. Astron. J. 121, 1050-1070 (2001). astro-ph/0010654. https://doi.org/10.1086/318769

S. Mateos, F.J. Carrera, X. Barcons, A. Alonso-Herrero, A. Hernán-Caballero, M. Page, C. Ramos Almeida, A. Caccianiga, T. Miyaji, A. Blain, Survival of the obscuring torus in the most powerful active galactic nuclei. Astrophys. J. Lett. 841, L18 (2017). 1705.04323. https://doi.org/10.3847/2041-8213/aa7268

T. Matheson, S. Blondin, R.J. Foley, R. Chornock, A.V. Filippenko, B. Leibundgut, R.C. Smith, J. Sollerman, J. Spyromilio, R.P. Kirshner, A. Clocchiatti, C. Aguilera, B. Barris, A.C. Becker, P. Challis, R. Covarrubias, P. Garnavich, M. Hicken, S. Jha, K. Krisciunas, W. Li, A. Miceli, G. Miknaitis, J.L. Prieto, A. Rest, A.G. Riess, M.E. Salvo, B.P. Schmidt, C.W. Stubbs, N.B. Suntzeff, J.L. Tonry, Spectroscopy of high-redshift supernovae from the ESSENCE project: the first 2 years. Astron. J. 129, 2352-2375 (2005). astro-ph/0411357. https://doi.org/10.1086/429679 
T. Matheson, X. Fan, R. Green, A. McConnachie, J. Newman, K. Olsen, P. Szkody, W.M. Wood-Vasey, Spectroscopy in the era of LSST. 1311.2496 (2013)

I.M. McHardy, D.T. Cameron, T. Dwelly, S. Connolly, P. Lira, D. Emmanoulopoulos, J. Gelbord, E. Breedt, P. Arevalo, P. Uttley, Swift monitoring of NGC 5548: X-ray reprocessing and short-term UV/optical variability. Mon. Not. R. Astron. Soc. 444, 1469-1474 (2014). 1407.6361. https://doi.org/10.1093/ mnras/stu 1636

S. Mei, J.P. Blakeslee, P. Côté, J.L. Tonry, M.J. West, L. Ferrarese, A. Jordán, E.W. Peng, A. Anthony, D. Merritt, The ACS Virgo Cluster survey, XIII: SBF distance catalog and the three-dimensional structure of the Virgo Cluster. Astrophys. J. 655, 144-162 (2007). astro-ph/0702510. https://doi.org/10.1086/509598

G. Miknaitis, G. Pignata, A. Rest, W.M. Wood-Vasey, S. Blondin, P. Challis, R.C. Smith, C.W. Stubbs, N.B. Suntzeff, R.J. Foley, T. Matheson, J.L. Tonry, C. Aguilera, J.W. Blackman, A.C. Becker, A. Clocchiatti, R. Covarrubias, T.M. Davis, A.V. Filippenko, A. Garg, P.M. Garnavich, M. Hicken, S. Jha, K. Krisciunas, R.P. Kirshner, B. Leibundgut, W. Li, A. Miceli, G. Narayan, J.L. Prieto, A.G. Riess, M.E. Salvo, B.P. Schmidt, J. Sollerman, J. Spyromilio, A. Zenteno, The ESSENCE supernova survey: survey optimization, observations, and supernova photometry. Astrophys. J. 666, 674-693 (2007). astro-ph/0701043. https://doi.org/10.1086/519986

R. Minkowski, Spectra of supernovae. Publ. Astron. Soc. Pac. 53, 224 (1941). https://doi.org/10.1086/ 125315

R.C. Mitchell, E. Baron, D. Branch, P.H. Hauschildt, P.E. Nugent, P. Lundqvist, S. Blinnikov, C.S.J. Pun, Detailed spectroscopic analysis of SN 1987A: the distance to the Large Magellanic Cloud using the spectral-fitting expanding atmosphere method. Astrophys. J. 574, 293-305 (2002). astro-ph/0204012. https://doi.org/10.1086/340928

M. Miyoshi, J. Moran, J. Herrnstein, L. Greenhill, N. Nakai, P. Diamond, M. Inoue, Evidence for a black hole from high rotation velocities in a sub-parsec region of NGC4258. Nature 373, 127-129 (1995). https://doi.org/10.1038/373127a0

J. Modzelewska, B. Czerny, K. Hryniewicz, M. Bilicki, M. Krupa, A. Świętoń, W. Pych, A. Udalski, T.P. Adhikari, F. Petrogalli, SALT long-slit spectroscopy of CTS C30.10: two-component Mg II line. Astron. Astrophys. 570, A53 (2014). 1408.1520. https://doi.org/10.1051/0004-6361/201424332

A.J. Monson, R.L. Beaton, V. Scowcroft, W.L. Freedman, B.F. Madore, J.A. Rich, M. Seibert, J.A. Kollmeier, G. Clementini, Standard galactic field RR Lyrae, I: optical to mid-infrared phased photometry. Astron. J. 153, 96 (2017). 1703.01520. https://doi.org/10.3847/1538-3881/153/3/96

R. Nair, S. Jhingan, D. Jain, Observational cosmology and the cosmic distance duality relation. J. Cosmol. Astropart. Phys. 5, 023 (2011). 1102.1065. https://doi.org/10.1088/1475-7516/2011/05/023

G. Nelemans, S. Toonen, M. Bours, Theoretical delay time distributions, in Binary Paths to Type Ia Supernovae Explosions, ed. by R. Di Stefano, M. Orio, M. Moe. IAU Symposium, vol. 281 (2013), pp. 225231. 1204.2960. https://doi.org/10.1017/S1743921312015098

M.T.B. Nielsen, R. Voss, G. Nelemans, Upper limits on bolometric luminosities of 10 type Ia supernova progenitors from Chandra observations. Mon. Not. R. Astron. Soc. 426, 2668-2676 (2012). 1109.6605. https://doi.org/10.1111/j.1365-2966.2012.21596.x

M.T.B. Nielsen, C. Dominik, G. Nelemans, R. Voss, Obscuration of supersoft X-ray sources by circumbinary material. A way to hide type Ia supernova progenitors? Astron. Astrophys. 549, A32 (2013). 1207.6310. https://doi.org/10.1051/0004-6361/201219195

M. Nikolajuk, I.E. Papadakis, B. Czerny, Black hole mass estimation from X-ray variability measurements in active galactic nuclei. Mon. Not. R. Astron. Soc. 350, L26-L30 (2004). astro-ph/0403326. https://doi.org/10.1111/j.1365-2966.2004.07829.x

S. Nissanke, D.E. Holz, S.A. Hughes, N. Dalal, J.L. Sievers, Exploring short gamma-ray bursts as gravitational-wave standard sirens. Astrophys. J. 725, 496-514 (2010). 0904.1017. https://doi.org/ 10.1088/0004-637X/725/1/496

S. Nissanke, D.E. Holz, N. Dalal, S.A. Hughes, J.L. Sievers, C.M. Hirata, Determining the Hubble constant from gravitational wave observations of merging compact binaries. 1307.2638 (2013)

K. Nomoto, Y. Kondo, Conditions for accretion-induced collapse of white dwarfs. Astrophys. J. Lett. 367, L19-L22 (1991). https://doi.org/10.1086/185922

P. Nugent, M. Sullivan, R. Ellis, A. Gal-Yam, D.C. Leonard, D.A. Howell, P. Astier, R.G. Carlberg, A. Conley, S. Fabbro, D. Fouchez, J.D. Neill, R. Pain, K. Perrett, C.J. Pritchet, N. Regnault, Toward a cosmological Hubble diagram for type II-P supernovae. Astrophys. J. 645, 841-850 (2006). astro-ph/0603535. https://doi.org/10.1086/504413

J.E. O’Donnell, $R_{n u}$-dependent optical and near-ultraviolet extinction. Astrophys. J. 422, 158-163 (1994). https://doi.org/10.1086/173713

V.L. Oknyanskij, On the possibility for measuring the Hubble constant from optical-to-NIR variability time delay in AGNs. Odessa Astron. Publ. 12, 99 (1999) 
E.F. Olivares, M. Hamuy, G. Pignata, J. Maza, M. Bersten, M.M. Phillips, N.B. Suntzeff, A.V. Filippenko, N.I. Morrel, R.P. Kirshner, T. Matheson, The standardized candle method for type II Plateau supernovae. Astrophys. J. 715, 833-853 (2010). 1004.2534. https://doi.org/10.1088/0004-637X/715/2/833

B. Paczynski, Gamma-ray bursters at cosmological distances. Astrophys. J. Lett. 308, L43-L46 (1986). https://doi.org/10.1086/184740

R. Pakmor, M. Kromer, F.K. Röpke, S.A. Sim, A.J. Ruiter, W. Hillebrandt, Sub-luminous type Ia supernovae from the mergers of equal-mass white dwarfs with mass $\sim 0.9 M_{\text {solar }}$. Nature 463, 61-64 (2010). 0911.0926. https://doi.org/10.1038/nature08642

R. Pakmor, M. Kromer, S. Taubenberger, V. Springel, Helium-ignited violent mergers as a unified model for normal and rapidly declining type Ia supernovae. Astrophys. J. Lett. 770, L8 (2013). 1302.2913. https://doi.org/10.1088/2041-8205/770/1/L8

J. Parrent, B. Friesen, M. Parthasarathy, A review of type Ia supernova spectra. Astrophys. Space Sci. 351, 1-52 (2014). 1402.6337. https://doi.org/10.1007/s10509-014-1830-1

J.T. Parrent, D. Sand, S. Valenti, M.J. Graham, D.A. Howell, Spectroscopic confirmation and classification of PSN J14323388-4413278 in NGC 5643. Astron. (2013). Telegr. 4817

F. Patat, S. Benetti, S. Justham, P.A. Mazzali, L. Pasquini, E. Cappellaro, M. Della Valle, P. -Podsiadlowski, M. Turatto, A. Gal-Yam, J.D. Simon, Upper limit for circumstellar gas around the type Ia SN 2000cx. Astron. Astrophys. 474, 931-936 (2007). 0708.3698. https://doi.org/10.1051/0004-6361:20078393

F. Patat, S. Taubenberger, N.L.J. Cox, D. Baade, A. Clocchiatti, P. Höflich, J.R. Maund, E. Reilly, J. Spyromilio, L. Wang, J.C. Wheeler, P. Zelaya, Properties of extragalactic dust inferred from linear polarimetry of type Ia supernovae. Astron. Astrophys. 577, A53 (2015). 1407.0136. https://doi.org/10.1051/ 0004-6361/201424507

S. Perlmutter, S. Gabi, G. Goldhaber, A. Goobar, D.E. Groom, I.M. Hook, A.G. Kim, M.Y. Kim, J.C. Lee, R. Pain, C.R. Pennypacker, I.A. Small, R.S. Ellis, R.G. McMahon, B.J. Boyle, P.S. Bunclark, D. Carter, M.J. Irwin, K. Glazebrook, H.J.M. Newberg, A.V. Filippenko, T. Matheson, M. Dopita, W.J. Couch, Measurements of the cosmological parameters $\Omega$ and $\Lambda$ from the first seven supernovae at $z \geq 0.35$. Astrophys. J. 483, 565-581 (1997). astro-ph/9608192. https://doi.org/10.1086/304265

S. Perlmutter, G. Aldering, M. della Valle, S. Deustua, R.S. Ellis, S. Fabbro, A. Fruchter, G. Goldhaber, D.E. Groom, I.M. Hook, A.G. Kim, M.Y. Kim, R.A. Knop, C. Lidman, R.G. McMahon, P. Nugent, R. Pain, N. Panagia, C.R. Pennypacker, P. Ruiz-Lapuente, B. Schaefer, N. Walton, Discovery of a supernova explosion at half the age of the universe. Nature 391, 51 (1998). astro-ph/9712212. https://doi.org/10.1038/34124

S. Perlmutter, G. Aldering, G. Goldhaber, R.A. Knop, P. Nugent, P.G. Castro, S. Deustua, S. Fabbro, A. Goobar, D.E. Groom, I.M. Hook, A.G. Kim, M.Y. Kim, J.C. Lee, N.J. Nunes, R. Pain, C.R. Pennypacker, R. Quimby, C. Lidman, R.S. Ellis, M. Irwin, R.G. McMahon, P. Ruiz-Lapuente, N. Walton, B. Schaefer, B.J. Boyle, A.V. Filippenko, T. Matheson, A.S. Fruchter, N. Panagia, H.J.M. Newberg, W.J. Couch, T.S.C. Project, Measurements of $\Omega$ and $\Lambda$ from 42 high-redshift supernovae. Astrophys. J. 517, 565-586 (1999a). astro-ph/9812133. https://doi.org/10.1086/307221

S. Perlmutter, G. Aldering, G. Goldhaber, R.A. Knop, P. Nugent, P.G. Castro, S. Deustua, S. Fabbro, A. Goobar, D.E. Groom, I.M. Hook, A.G. Kim, M.Y. Kim, J.C. Lee, N.J. Nunes, R. Pain, C.R. Pennypacker, R. Quimby, C. Lidman, R.S. Ellis, M. Irwin, R.G. McMahon, P. Ruiz-Lapuente, N. Walton, B. Schaefer, B.J. Boyle, A.V. Filippenko, T. Matheson, A.S. Fruchter, N. Panagia, H.J.M. Newberg, W.J. Couch, T.S.C. Project, Measurements of $\Omega$ and $\Lambda$ from 42 high-redshift supernovae. Astrophys. J. 517, 565-586 (1999b). astro-ph/9812133. https://doi.org/10.1086/307221

S. Perlmutter, M.S. Turner, M. White, Constraining dark energy with type Ia supernovae and largescale structure. Phys. Rev. Lett. 83, 670-673 (1999c). astro-ph/9901052. https://doi.org/10.1103/ PhysRevLett.83.670

B.M. Peterson, Measuring the masses of supermassive black holes. Space Sci. Rev. 183, 253-275 (2014a). https://doi.org/10.1007/s11214-013-9987-4

B.M. Peterson, Measuring the masses of supermassive black holes. Space Sci. Rev. 183, 253-275 (2014b). https://doi.org/10.1007/s11214-013-9987-4

B.M. Peterson, A.J. Barth, P. Berlind, R. Bertram, K. Bischoff, N.G. Bochkarev, A.N. Burenkov, F.Z. Cheng, M. Dietrich, A.V. Filippenko, E. Giannuzzo, L.C. Ho, J.P. Huchra, J. Hunley, S. Kaspi, W. Kollatschny, D.C. Leonard, Y.F. Malkov, T. Matheson, M. Mignoli, B. Nelson, P. Papaderos, J. Peters, R.W. Pogge, V.I. Pronik, S.G. Sergeev, E.A. Sergeeva, A.I. Shapovalova, G.M. Stirpe, S. Tokarz, R.M. Wagner, I. Wanders, J.Y. Wei, B.J. Wilkes, H. Wu, S.J. Xue, Z.L. Zou, Steps toward determination of the size and structure of the broad-line region in active galactic nuclei, XV: long-term optical monitoring of NGC 5548. Astrophys. J. 510, 659-668 (1999). astro-ph/9808236. https://doi.org/10.1086/306604

B.M. Peterson, L. Ferrarese, K.M. Gilbert, S. Kaspi, M.A. Malkan, D. Maoz, D. Merritt, H. Netzer, C.A. Onken, R.W. Pogge, M. Vestergaard, A. Wandel, Central masses and broad-line region sizes of active 
galactic nuclei, II: a homogeneous analysis of a large reverberation-mapping database. Astrophys. J. 613, 682-699 (2004). astro-ph/0407299. https://doi.org/10.1086/423269

M.M. Phillips, The absolute magnitudes of type IA supernovae. Astrophys. J. Lett. 413, L105-L108 (1993). https://doi.org/10.1086/186970

M.M. Phillips, Near-infrared properties of type Ia supernovae. Publ. Astron. Soc. Aust. 29, 434-446 (2012). 1111.4463. https://doi.org/10.1071/AS11056

M.M. Phillips, P. Lira, N.B. Suntzeff, R.A. Schommer, M. Hamuy, J. Maza, The reddening-free decline rate versus luminosity relationship for type IA supernovae. Astron. J. 118, 1766-1776 (1999). https://doi.org/10.1086/301032

M.M. Phillips, J.D. Simon, N. Morrell, C.R. Burns, N.L.J. Cox, R.J. Foley, A.I. Karakas, F. Patat, A. Sternberg, R.E. Williams, A. Gal-Yam, E.Y. Hsiao, D.C. Leonard, S.E. Persson, M. Stritzinger, I.B. Thompson, A. Campillay, C. Contreras, G. Folatelli, W.L. Freedman, M. Hamuy, M. Roth, G.A. Shields, N.B. Suntzeff, L. Chomiuk, I.I. Ivans, B.F. Madore, B.E. Penprase, D. Perley, G. Pignata, G. Preston, A.M. Soderberg, On the source of the dust extinction in type Ia supernovae and the discovery of anomalously strong Na I absorption. Astrophys. J. 779, 38 (2013). 1311.0147. https://doi.org/ 10.1088/0004-637X/779/1/38

L. Piersanti, A. Tornambé, L.R. Yungelson, He-accreting white dwarfs: accretion regimes and final outcomes. Mon. Not. R. Astron. Soc. 445, 3239-3262 (2014). 1409.3589. https://doi.org/10.1093/mnras/stu 1885

G. Pietrzyński, D. Graczyk, W. Gieren, I.B. Thompson, B. Pilecki, A. Udalski, I. Soszyński, S. Kozłowski, P. Konorski, K. Suchomska, G. Bono, P.G.P. Moroni, S. Villanova, N. Nardetto, F. Bresolin, R.P. Kudritzki, J. Storm, A. Gallenne, R. Smolec, D. Minniti, M. Kubiak, M.K. Szymański, R. Poleski, Ł. Wyrzykowski, K. Ulaczyk, P. Pietrukowicz, M. Górski, P. Karczmarek, An eclipsing-binary distance to the Large Magellanic Cloud accurate to two per cent. Nature 495, 76-79 (2013). 1303.2063. https://doi.org/10.1038/nature11878

F.A.E. Pirani, On the physical significance of the Riemann tensor. Acta Phys. Pol. 15, 389-405 (1956)

Planck Collaboration, P.A.R. Ade, N. Aghanim, M. Arnaud, M. Ashdown, J. Aumont, C. Baccigalupi, A.J. Banday, R.B. Barreiro, J.G. Bartlett et al. Planck 2015 results, XIII: cosmological parameters. Astron. Astrophys. 594, A13 (2016). 1502.01589. https://doi.org/10.1051/0004-6361/201525830

J. Polshaw, R. Kotak, K.C. Chambers, S.J. Smartt, S. Taubenberger, M. Kromer, E.E.E. Gall, W. Hillebrandt, M. Huber, K.W. Smith, R.J. Wainscoat, A supernova distance to the anchor galaxy NGC 4258. Astron. Astrophys. 580, L15 (2015). 1509.00507. https://doi.org/10.1051/0004-6361/201526902

J. Polshaw, R. Kotak, L. Dessart, M. Fraser, A. Gal-Yam, C. Inserra, S.A. Sim, S.J. Smartt, J. Sollerman, C. Baltay, D. Rabinowitz, S. Benetti, M.T. Botticella, H. Campbell, T.W. Chen, L. Galbany, R. McKinnon, M. Nicholl, K.W. Smith, M. Sullivan, K. Takáts, S. Valenti, D.R. Young, LSQ13fn: A type II-Plateau supernova with a possibly low metallicity progenitor that breaks the standardised candle relation. Astron. Astrophys. 588, A1 (2016). 1511.01718. https://doi.org/10.1051/0004-6361/201527682

D. Poznanski, N. Butler, A.V. Filippenko, M. Ganeshalingam, W. Li, J.S. Bloom, R. Chornock, R.J. Foley, P.E. Nugent, J.M. Silverman, S.B. Cenko, E.L. Gates, D.C. Leonard, A.A. Miller, M. Modjaz, F.J.D. Serduke, N. Smith, B.J. Swift, D.S. Wong, Improved standardization of type II-P supernovae: application to an expanded sample. Astrophys. J. 694, 1067-1079 (2009). 0810.4923. https://doi.org/10.1088/0004-637X/694/2/1067

I.P. Pskovskii, Light curves, color curves, and expansion velocity of type I supernovae as functions of the rate of brightness decline. Sov. Astron. 21, 675-682 (1977)

M.L. Pumo, L. Zampieri, Radiation-hydrodynamical modeling of core-collapse supernovae: light curves and the evolution of photospheric velocity and temperature. Astrophys. J. 741, 41 (2011). 1108.0688. https://doi.org/10.1088/0004-637X/741/1/41

E.D. Reese, J.E. Carlstrom, M. Joy, J.J. Mohr, L. Grego, W.L. Holzapfel, Determining the cosmic distance scale from interferometric measurements of the Sunyaev-Zeldovich effect. Astrophys. J. 581, 53-85 (2002). astro-ph/0205350. https://doi.org/10.1086/344137

A.G. Riess, W.H. Press, R.P. Kirshner, Determining the motion of the local group using type IA supernovae light curve shapes. Astrophys. J. Lett. 445, L91-L94 (1995). astro-ph/9412017. https://doi.org/ $10.1086 / 187897$

A.G. Riess, W.H. Press, R.P. Kirshner, A precise distance indicator: type IA supernova multicolor light-curve shapes. Astrophys. J. 473, 88 (1996). astro-ph/9604143. https://doi.org/10.1086/178129

A.G. Riess, A.V. Filippenko, P. Challis, A. Clocchiatti, A. Diercks, P.M. Garnavich, R.L. Gilliland, C.J. Hogan, S. Jha, R.P. Kirshner, B. Leibundgut, M.M. Phillips, D. Reiss, B.P. Schmidt, R.A. Schommer, R.C. Smith, J. Spyromilio, C. Stubbs, N.B. Suntzeff, J. Tonry, Observational evidence from supernovae for an accelerating universe and a cosmological constant. Astron. J. 116, 1009-1038 (1998a). astro-ph/9805201. https://doi.org/10.1086/300499

A.G. Riess, A.V. Filippenko, P. Challis, A. Clocchiatti, A. Diercks, P.M. Garnavich, R.L. Gilliland, C.J. Hogan, S. Jha, R.P. Kirshner, B. Leibundgut, M.M. Phillips, D. Reiss, B.P. Schmidt, R.A. Schommer, 
R.C. Smith, J. Spyromilio, C. Stubbs, N.B. Suntzeff, J. Tonry, Observational evidence from supernovae for an accelerating universe and a cosmological constant. Astron. J. 116, 1009-1038 (1998b). astro-ph/9805201. https://doi.org/10.1086/300499

A.G. Riess, R.P. Kirshner, B.P. Schmidt, S. Jha, P. Challis, P.M. Garnavich, A.A. Esin, C. Carpenter, R. Grashius, R.E. Schild, P.L. Berlind, J.P. Huchra, C.F. Prosser, E.E. Falco, P.J. Benson, C. Briceño, W.R. Brown, N. Caldwell, I.P.dell'Antonio, A.V. Filippenko, A.A. Goodman, N.A. Grogin, T. Groner, J.P. Hughes, P.J. Green, R.A. Jansen, J.T. Kleyna, J.X. Luu, L.M. Macri, B.A. McLeod, K.K. McLeod, B.R. McNamara, B. McLean, A.A.E. Milone, J.J. Mohr, D. Moraru, C. Peng, J. Peters, A.H. Prestwich, K.Z. Stanek, A. Szentgyorgyi, P. Zhao, BVRI light curves for 22 type IA supernovae. Astron. J. 117, 707-724 (1999). astro-ph/9810291. https://doi.org/10.1086/300738

A.G. Riess, P.E. Nugent, R.L. Gilliland, B.P. Schmidt, J. Tonry, M. Dickinson, R.I. Thompson, T. Budavári, S. Casertano, A.S. Evans, A.V. Filippenko, M. Livio, D.B. Sanders, A.E. Shapley, H. Spinrad, C.C. Steidel, D. Stern, J. Surace, S. Veilleux, The farthest known supernova: support for an accelerating universe and a glimpse of the epoch of deceleration. Astrophys. J. 560, 49-71 (2001). astro-ph/0104455. https://doi.org/10.1086/322348

A.G. Riess, L.G. Strolger, J. Tonry, S. Casertano, H.C. Ferguson, B. Mobasher, P. Challis, A.V. Filippenko, S. Jha, W. Li, R. Chornock, R.P. Kirshner, B. Leibundgut, M. Dickinson, M. Livio, M. Giavalisco, C.C. Steidel, T. Benítez, Z. Tsvetanov, Type Ia supernova discoveries at $z>1$ from the Hubble Space Telescope: evidence for past deceleration and constraints on dark energy evolution. Astrophys. J. 607, 665-687 (2004a). astro-ph/0402512. https://doi.org/10.1086/383612

A.G. Riess, L.G. Strolger, J. Tonry, Z. Tsvetanov, S. Casertano, H.C. Ferguson, B. Mobasher, P. Challis, N. Panagia, A.V. Filippenko, W. Li, R. Chornock, R.P. Kirshner, B. Leibundgut, M. Dickinson, A. Koekemoer, N.A. Grogin, M. Giavalisco, Identification of type Ia supernovae at redshift 1.3 and beyond with the advanced camera for surveys on the Hubble Space Telescope. Astrophys. J. Lett. 600, L163L166 (2004b). astro-ph/0308185. https://doi.org/10.1086/378311

A.G. Riess, L.G. Strolger, S. Casertano, H.C. Ferguson, B. Mobasher, B. Gold, P.J. Challis, A.V. Filippenko, S. Jha, W. Li, J. Tonry, R. Foley, R.P. Kirshner, M. Dickinson, E. MacDonald, D. Eisenstein, M. Livio, J. Younger, C. Xu, T. Dahlén, D. Stern, New Hubble Space Telescope discoveries of type Ia supernovae at $z \geq 1$ : narrowing constraints on the early behavior of dark energy. Astrophys. J. 659, 98-121 (2007). astro-ph/0611572. https://doi.org/10.1086/510378

A.G. Riess, L. Macri, S. Casertano, M. Sosey, H. Lampeitl, H.C. Ferguson, A.V. Filippenko, S.W. Jha, W. Li, R. Chornock, D. Sarkar, A redetermination of the Hubble constant with the Hubble Space Telescope from a differential distance ladder. Astrophys. J. 699, 539-563 (2009). 0905.0695. https://doi.org/ 10.1088/0004-637X/699/1/539

A.G. Riess, L. Macri, S. Casertano, H. Lampeitl, H.C. Ferguson, A.V. Filippenko, S.W. Jha, W. Li, R. Chornock, A 3\% solution: determination of the Hubble constant with the Hubble Space Telescope and Wide Field Camera 3. Astrophys. J. 730, 119 (2011). 1103.2976. https://doi.org/10.1088/ 0004-637X/730/2/119

A.G. Riess, L.M. Macri, S.L. Hoffmann, D. Scolnic, S. Casertano, A.V. Filippenko, B.E. Tucker, M.J. Reid, D.O. Jones, J.M. Silverman, R. Chornock, P. Challis, W. Yuan, P.J. Brown, R.J. Foley, A 2.4\% determination of the local value of the Hubble constant. Astrophys. J. 826, 56 (2016). 1604.01424. https://doi.org/10.3847/0004-637X/826/1/56

A.G. Riess, S.A. Rodney, D.M. Scolnic, D.L. Shafer, L.G. Strolger, H.C. Ferguson, M. Postman, O. Graur, D. Maoz, S.W. Jha, B. Mobasher, S. Casertano, B. Hayden, A. Molino, J. Hjorth, P.M. Garnavich, D.O. Jones, R.P. Kirshner, A.M. Koekemoer, N.A. Grogin, G. Brammer, S. Hemmati, M. Dickinson, P.M. Challis, S. Wolff, K.I. Clubb, A.V. Filippenko, H. Nayyeri, U. Vivian, D.C. Koo, S.M. Faber, D. Kocevski, L. Bradley, D. Coe, Type Ia supernova distances at $z>1.5$ from the Hubble Space Telescope multi-cycle treasury programs: the early expansion rate. 1710.00844 (2017)

M. Rigault, Evidence of environmental dependencies of type Ia supernovae from the nearby supernova factory indicated by local H $\alpha$. Astron. Astrophys. 560, A66 (2013). 1309.1182. https://doi.org/ $10.1051 / 0004-6361 / 201322104$

M. Rigault, G. Aldering, M. Kowalski, Y. Copin, P. Antilogus, C. Aragon, S. Bailey, C. Baltay, D. Baugh, S. Bongard, K. Boone, C. Buton, J. Chen, N. Chotard, H.K. Fakhouri, U. Feindt, P. Fagrelius, M. Fleury, D. Fouchez, E. Gangler, B. Hayden, A.G. Kim, P.F. Leget, S. Lombardo, J. Nordin, R. Pain, E. Pecontal, R. Pereira, S. Perlmutter, D. Rabinowitz, K. Runge, D. Rubin, C. Saunders, G. Smadja, C. Sofiatti, N. Suzuki, C. Tao, B.A. Weaver, Confirmation of a star formation bias in type Ia supernova distances and its effect on the measurement of the Hubble constant. Astrophys. J. 802, 20 (2015). 1412.6501. https://doi.org/10.1088/0004-637X/802/1/20

G. Risaliti, E. Lusso, A Hubble diagram for quasars. Astrophys. J. 815, 33 (2015). 1505.07118. https:// doi.org/10.1088/0004-637X/815/1/33 
A.M. Ritchey, D.E. Welty, J.A. Dahlstrom, D.G. York, Diffuse atomic and molecular gas in the interstellar medium of m82 toward sn 2014j. Astrophys. J. 799, 197 (2015). 1407.5723. https://doi.org/ 10.1088/0004-637X/799/2/197

I. Robinson, A. Trautman, Spherical gravitational waves. Phys. Rev. Lett. 4, 431-432 (1960). https://doi.org/ 10.1103/PhysRevLett.4.431

F.K. Röpke, I.R. Seitenzahl, S. Benitez, M. Fink, R. Pakmor, M. Kromer, S.A. Sim, F. Ciaraldi-Schoolmann, W. Hillebrandt, Modeling type Ia supernova explosions. Prog. Part. Nucl. Phys. 66, 309-318 (2011). https://doi.org/10.1016/j.ppnp.2011.01.026

D. Rubin, R.A. Knop, E. Rykoff, G. Aldering, R. Amanullah, K. Barbary, M.S. Burns, A. Conley, N. Connolly, S. Deustua, V. Fadeyev, H.K. Fakhouri, A.S. Fruchter, R.A. Gibbons, G. Goldhaber, A. Goobar, E.Y. Hsiao, X. Huang, M. Kowalski, C. Lidman, J. Meyers, J. Nordin, S. Perlmutter, C. Saunders, A.L. Spadafora, V. Stanishev, N. Suzuki, L. Wang, Precision measurement of the most distant spectroscopically confirmed supernova Ia with the Hubble Space Telescope. Supernova Cosmology Project T 763, 35 (2013). Astrophys. J.. 1205.3494. https://doi.org/10.1088/0004-637X/763/1/35

A.J. Ruiter, S.A. Sim, R. Pakmor, M. Kromer, I.R. Seitenzahl, K. Belczynski, M. Fink, M. Herzog, W. Hillebrandt, F.K. Röpke, S. Taubenberger, On the brightness distribution of type Ia supernovae from violent white dwarf mergers. Mon. Not. R. Astron. Soc. 429, 1425-1436 (2013). 1209.0645. https://doi.org/10.1093/mnras/sts423

P. Ruiz-Lapuente, F. Comeron, J. Méndez, R. Canal, S.J. Smartt, A.V. Filippenko, R.L. Kurucz, R. Chornock, R.J. Foley, V. Stanishev, R. Ibata, The binary progenitor of Tycho Brahe's 1572 supernova. Nature 431, 1069-1072 (2004). astro-ph/0410673. https://doi.org/10.1038/nature03006

C.L. Sarazin, X-ray emission from clusters of galaxies (1988)

M. Sasdelli, E.E.O. Ishida, W. Hillebrandt, C. Ashall, P.A. Mazzali, S.J. Prentice, Breaking the colourreddening degeneracy in type Ia supernovae. Mon. Not. R. Astron. Soc. 460, 373-382 (2016). 1604.03899. https://doi.org/10.1093/mnras/stw900

B. Sathyaprakash, B.F. Schutz, Physics, astrophysics and cosmology with gravitational waves. Living Rev. Relativ. 12, 2 (2009). https://doi.org/10.1007//rr-2009-2

B.E. Schaefer, The Hubble diagram to redshift $>6$ from 69 gamma-ray bursts. Astrophys. J. 660, 16-46 (2007). astro-ph/0612285. https://doi.org/10.1086/511742

B.E. Schaefer, A. Pagnotta, An absence of ex-companion stars in the type Ia supernova remnant SNR 050967.5. Nature 481, 164-166 (2012). https://doi.org/10.1038/nature10692

E.F. Schlafly, D.P. Finkbeiner, Measuring reddening with Sloan digital sky survey stellar spectra and recalibrating SFD. Astrophys. J. 737, 103 (2011). 1012.4804. https://doi.org/10.1088/0004-637X/737/2/103

D.J. Schlegel, D.P. Finkbeiner, M. Davis, Maps of dust infrared emission for use in estimation of reddening and cosmic microwave background radiation foregrounds. Astrophys. J. 500, 525-553 (1998). astro-ph/9710327. https://doi.org/10.1086/305772

B.P. Schmidt, N.B. Suntzeff, M.M. Phillips, R.A. Schommer, A. Clocchiatti, R.P. Kirshner, P. Garnavich, P. Challis, B. Leibundgut, J. Spyromilio, A.G. Riess, A.V. Filippenko, M. Hamuy, R.C. Smith, C. Hogan, C. Stubbs, A. Diercks, D. Reiss, R. Gilliland, J. Tonry, J. Maza, A. Dressler, J. Walsh, R. Ciardullo, The high-Z supernova search: measuring cosmic deceleration and global curvature of the universe using type IA supernovae. Astrophys. J. 507, 46-63 (1998a). astro-ph/9805200. https://doi.org/10.1086/306308

B.P. Schmidt, N.B. Suntzeff, M.M. Phillips, R.A. Schommer, A. Clocchiatti, R.P. Kirshner, P. Garnavich, P. Challis, B. Leibundgut, J. Spyromilio, A.G. Riess, A.V. Filippenko, M. Hamuy, R.C. Smith, C. Hogan, C. Stubbs, A. Diercks, D. Reiss, R. Gilliland, J. Tonry, J. Maza, A. Dressler, J. Walsh, R. Ciardullo, The high-Z supernova search: measuring cosmic deceleration and global curvature of the universe using type IA supernovae. Astrophys. J. 507, 46-63 (1998b). astro-ph/9805200. https://doi.org/10.1086/306308

B.F. Schutz, Determining the Hubble constant from gravitational wave observations. Nature 323, 310 (1986). https://doi.org/10.1038/323310a0

B.F. Schutz, Lighthouses of gravitational wave astronomy, in Lighthouses of the Universe: The Most Luminous Celestial Objects and Their Use for Cosmology, ed. by M. Gilfanov, R. Sunyeav, E. Churazov (2002), p. 207. gr-qc/0111095. https://doi.org/10.1007/10856495_29

B.F. Schutz, Networks of gravitational wave detectors and three figures of merit. Class. Quantum Gravity 28(12), 125023 (2011). 1102.5421. https://doi.org/10.1088/0264-9381/28/12/125023

D.M. Scolnic, A.G. Riess, R.J. Foley, A. Rest, S.A. Rodney, D.J. Brout, D.O. Jones, Color dispersion and Milky-Way-like reddening among type Ia supernovae. Astrophys. J. 780, 37 (2014). 1306.4050. https://doi.org/10.1088/0004-637X/780/1/37

M. Sereno, E. De Filippis, G. Longo, M.W. Bautz, Measuring the three-dimensional structure of galaxy clusters, II: are clusters of galaxies oblate or prolate? Astrophys. J. 645, 170-178 (2006). astro-ph/0602051. https://doi.org/10.1086/503198 
S.G. Sergeev, V.T. Doroshenko, Y.V. Golubinskiy, N.I. Merkulova, E.A. Sergeeva, Lag-luminosity relationship for interband lags between variations in B, V, R, and I bands in active galactic nuclei. Astrophys. J. 622, 129-135 (2005). https://doi.org/10.1086/427820

N.I. Shakura, R.A. Sunyaev, Black holes in binary systems. Observational appearance. Astron. Astrophys. 24, 337-355 (1973)

H. Shapley, On the existence of external galaxies. Publ. Astron. Soc. Pac. 31, 261 (1919). https://doi.org/ 10.1086/122870

B.J. Shappee, J.L. Prieto, D. Grupe, C.S. Kochanek, K.Z. Stanek, G. De Rosa, S. Mathur, Y. Zu, B.M. Peterson, R.W. Pogge, S. Komossa, M. Im, J. Jencson, T.W.S. Holoien, U. Basu, J.F. Beacom, D.M. Szczygieł, J. Brimacombe, S. Adams, A. Campillay, C. Choi, C. Contreras, M. Dietrich, M. Dubberley, M. Elphick, S. Foale, M. Giustini, C. Gonzalez, E. Hawkins, D.A. Howell, E.Y. Hsiao, M. Koss, K.M. Leighly, N. Morrell, D. Mudd, D. Mullins, J.M. Nugent, J. Parrent, M.M. Phillips, G. Pojmanski, W. Rosing, R. Ross, D. Sand, D.M. Terndrup, S. Valenti, Z. Walker, Y. Yoon, The man behind the curtain: X-rays drive the UV through NIR variability in the 2013 active galactic nucleus outburst in NGC 2617. Astrophys. J. 788, 48 (2014a). 1310.2241. https://doi.org/10.1088/0004-637X/788/1/48

B.J. Shappee, J.L. Prieto, D. Grupe et al., The man behind the curtain: X-rays drive the UV through NIR variability in the 2013 active galactic nucleus outburst in NGC 2617. Astrophys. J. 788, 48 (2014b). 1310.2241. https://doi.org/10.1088/0004-637X/788/1/48

B.J. Shappee, K.Z. Stanek, C.S. Kochanek, P.M. Garnavich, Whimper of a bang: documenting the final days of the nearby type Ia supernova 2011fe. Astrophys. J. 841, 48 (2017). 1608.01155. https://doi.org/ 10.3847/1538-4357/aa6eab

K.J. Shen, L. Bildsten, D. Kasen, E. Quataert, The long-term evolution of double white dwarf mergers. Astrophys. J. 748, 35 (2012). 1108.4036. https://doi.org/10.1088/0004-637X/748/1/35

Y. Shen, K. Horne, C.J. Grier, B.M. Peterson, K.D. Denney, J.R. Trump, M. Sun, W.N. Brandt, C.S. Kochanek, K.S. Dawson, P.J. Green, J.E. Greene, P.B. Hall, L.C. Ho, L. Jiang, K. Kinemuchi, I.D. McGreer, P. Petitjean, G.T. Richards, D.P. Schneider, M.A. Strauss, C. Tao, W.M. Wood-Vasey, Y. Zu, K. Pan, D. Bizyaev, J. Ge, D. Oravetz, A. Simmons, The Sloan digital sky survey reverberation mapping project: first broad-line $\mathrm{H} \beta$ and $\mathrm{Mg}$ II lags at $z$ above 0.3 from six-month spectroscopy. Astrophys. J. 818, 30 (2016). 1510.02802. https://doi.org/10.3847/0004-637X/818/1/30

L.P. Singer, H.Y. Chen, D.E. Holz, W.M. Farr, L.R. Price, V. Raymond, S.B. Cenko, N. Gehrels, J. Cannizzo, M.M. Kasliwal, S. Nissanke, M. Coughlin, B. Farr, A.L. Urban, S. Vitale, J. Veitch, P. Graff, C.P.L. Berry, S. Mohapatra, I. Mandel, Going the distance: mapping host galaxies of LIGO and Virgo sources in three dimensions using local cosmography and targeted follow-up. Astrophys. J. Lett. 829, L15 (2016). 1603.07333. https://doi.org/10.3847/2041-8205/829/1/L15

S.J. Smartt, Progenitors of core-collapse supernovae. Annu. Rev. Astron. Astrophys. 47, $63-106$ (2009). 0908.0700. https://doi.org/10.1146/annurev-astro-082708-101737

S.J. Smartt, J.J. Eldridge, R.M. Crockett, J.R. Maund, The death of massive stars, I: observational constraints on the progenitors of type II-P supernovae. Mon. Not. R. Astron. Soc. 395, 1409-1437 (2009). 0809.0403. https://doi.org/10.1111/j.1365-2966.2009.14506.x

D.A. Starkey, K. Horne, C. Villforth, Accretion disc time lag distributions: applying CREAM to simulated AGN light curves. Mon. Not. R. Astron. Soc. 456, 1960-1973 (2016). 1511.06162. https://doi.org/ 10.1093/mnras/stv2744

I. Steer, B.F. Madore, J.M. Mazzarella, M. Schmitz, H.G. Corwin, B.H.P. Chan, R. Ebert, G. Helou, K. Baker, X. Chen, C. Frayer, J. Jacobson, T. Lo, P. Ogle, O. Pevunova, S. Terek, Redshift-independent distances in the NASA/IPAC extragalactic database: methodology, content, and use of NED-D. Astron. J. 153, 37 (2017). 1612.09263. https://doi.org/10.3847/1538-3881/153/1/37

A.T. Steffen, I. Strateva, W.N. Brandt, D.M. Alexander, A.M. Koekemoer, B.D. Lehmer, D.P. Schneider, C. Vignali, The X-ray-to-optical properties of optically selected active galaxies over wide luminosity and redshift ranges. Astron. J. 131, 2826-2842 (2006). astro-ph/0602407. https://doi.org/10.1086/503627

A. Sternberg, A. Gal-Yam, J.D. Simon, D.C. Leonard, R.M. Quimby, M.M. Phillips, N. Morrell, I.B. Thompson, I. Ivans, J.L. Marshall, A.V. Filippenko, G.W. Marcy, J.S. Bloom, F. Patat, R.J. Foley, D. Yong, B.E. Penprase, D.J. Beeler, C. Allende Prieto, G.S. Stringfellow, Circumstellar material in type Ia supernovae via sodium absorption features. Science 333, 856 (2011). 1108.3664. https://doi.org/ 10.1126/science. 1203836

M. Stritzinger, C.R. Burns, M.M. Phillips, G. Folatelli, K. Krisciunas, S. Kattner, S.E. Persson, L. Boldt, A. Campillay, C. Contreras, W. Krzeminski, N. Morrell, F. Salgado, W.L. Freedman, M. Hamuy, B.F. Madore, M. Roth, N.B. Suntzeff, The distance to NGC 1316 (Fornax A) from observations of four type Ia supernovae. Astron. J. 140, 2036-2051 (2010). 1009.4390. https://doi.org/10.1088/ $0004-6256 / 140 / 6 / 2036$ 
J.W. Sulentic, P. Marziani, D. Dultzin, M. D’Onofrio, A. del Olmo, Fifty years of quasars: physical insights and potential for cosmology. J. Phys. Conf. Ser. 565, 012018 (2014). 1411.6900. https://doi.org/ 10.1088/1742-6596/565/1/012018

M. Sullivan, A. Conley, D.A. Howell, J.D. Neill, P. Astier, C. Balland, S. Basa, R.G. Carlberg, D. Fouchez, J. Guy, D. Hardin, I.M. Hook, R. Pain, N. Palanque-Delabrouille, K.M. Perrett, C.J. Pritchet, N. Regnault, J. Rich, V. Ruhlmann-Kleider, S. Baumont, E. Hsiao, T. Kronborg, C. Lidman, S. Perlmutter, E.S. Walker, The dependence of type Ia supernovae luminosities on their host galaxies. Mon. Not. R. Astron. Soc. 406, 782-802 (2010). 1003.5119. https://doi.org/10.1111/j.1365-2966.2010.16731.x

M. Sullivan, J. Guy, A. Conley, N. Regnault, P. Astier, C. Balland, S. Basa, R.G. Carlberg, D. Fouchez, D. Hardin, I.M. Hook, D.A. Howell, R. Pain, N. Palanque-Delabrouille, K.M. Perrett, C.J. Pritchet, J. Rich, V. Ruhlmann-Kleider, D. Balam, S. Baumont, R.S. Ellis, S. Fabbro, H.K. Fakhouri, N. Fourmanoit, S. González-Gaitán, M.L. Graham, M.J. Hudson, E. Hsiao, T. Kronborg, C. Lidman, A.M. Mourao, J.D. Neill, S. Perlmutter, P. Ripoche, N. Suzuki, E.S. Walker, SNLS3: constraints on dark energy combining the supernova legacy survey three-year data with other probes. Astrophys. J. 737, 102 (2011). 1104.1444. https://doi.org/10.1088/0004-637X/737/2/102

R.A. Sunyaev, Y.B. Zeldovich, The observations of relic radiation as a test of the nature of X-ray radiation from the clusters of galaxies. Comments Astrophys. Space Phys. 4, 173 (1972)

N. Suzuki, D. Rubin, C. Lidman, G. Aldering, R. Amanullah, K. Barbary, L.F. Barrientos, J. Botyanszki, M. Brodwin, N. Connolly, K.S. Dawson, A. Dey, M. Doi, M. Donahue, S. Deustua, P. Eisenhardt, E. Ellingson, L. Faccioli, V. Fadeyev, H.K. Fakhouri, A.S. Fruchter, D.G. Gilbank, M.D. Gladders, G. Goldhaber, A.H. Gonzalez, A. Goobar, A. Gude, T. Hattori, H. Hoekstra, E. Hsiao, X. Huang, Y. Ihara, M.J. Jee, D. Johnston, N. Kashikawa, B. Koester, K. Konishi, M. Kowalski, E.V. Linder, L. Lubin, J. Melbourne, J. Meyers, T. Morokuma, F. Munshi, C. Mullis, T. Oda, N. Panagia, S. Perlmutter, M. Postman, T. Pritchard, J. Rhodes, P. Ripoche, P. Rosati, D.J. Schlegel, A. Spadafora, S.A. Stanford, V. Stanishev, D. Stern, M. Strovink, N. Takanashi, K. Tokita, M. Wagner, L. Wang, N. Yasuda, H.K.C. Yee, Supernova Cosmology Project, The Hubble Space Telescope cluster supernova survey, V: improving the dark-energy constraints above $z>1$ and building an early-type-hosted supernova sample. Astrophys. J. 746, 85 (2012). . 1105.3470. https://doi.org/10.1088/0004-637X/746/1/85

H. Tananbaum, Y. Avni, G. Branduardi, M. Elvis, G. Fabbiano, E. Feigelson, R. Giacconi, J.P. Henry, J.P. Pye, A. Soltan, G. Zamorani, X-ray studies of quasars with the Einstein observatory. Astrophys. J. Lett. 234, L9-L13 (1979). https://doi.org/10.1086/183100

N.R. Tanvir, D.B. Fox, A.J. Levan, E. Berger, K. Wiersema, J.P.U. Fynbo, A. Cucchiara, T. Krühler, N. Gehrels, J.S. Bloom, J. Greiner, P.A. Evans, E. Rol, F. Olivares, J. Hjorth, P. Jakobsson, J. Farihi, R. Willingale, R.L.C. Starling, S.B. Cenko, D. Perley, J.R. Maund, J. Duke, R.A.M.J. Wijers, A.J. Adamson, A. Allan, M.N. Bremer, D.N. Burrows, A.J. Castro-Tirado, B. Cavanagh, A. de Ugarte Postigo, M.A. Dopita, T.A. Fatkhullin, A.S. Fruchter, R.J. Foley, J. Gorosabel, J. Kennea, T. Kerr, S. Klose, H.A. Krimm, V.N. Komarova, S.R. Kulkarni, A.S. Moskvitin, C.G. Mundell, T. Naylor, K. Page, B.E. Penprase, M. Perri, P. Podsiadlowski, K. Roth, R.E. Rutledge, T. Sakamoto, P. Schady, B.P. Schmidt, A.M. Soderberg, J. Sollerman, A.W. Stephens, G. Stratta, T.N. Ukwatta, D. Watson, E. Westra, T. Wold, A $\gamma$-ray burst at a redshift of $z \sim 8.2$. Nature 461, 1254-1257 (2009). 0906.1577. https://doi.org/10.1038/nature08459

L. Tartaglia, D. Sand, S. Wyatt, S. Valenti, K.A. Bostroem, D.E. Reichart, V. Haislip, V. Kouprianov, The discovery of DLT17u/AT 2017cbv. Astron. Telegr. 100158 (2017)

F. Thim, J.G. Hoessel, A. Saha, J. Claver, A. Dolphin, G.A. Tammann, Cepheids and long-period variables in NGC 4395. Astron. J. 127, 2322-2343 (2004). astro-ph/0401558. https://doi.org/10.1086/382244

J. Tonry, D.P. Schneider, A new technique for measuring extragalactic distances. Astron. J. 96, 807-815 (1988). https://doi.org/10.1086/114847

J.L. Tonry, A. Dressler, J.P. Blakeslee, E.A. Ajhar, A.B. Fletcher, G.A. Luppino, M.R. Metzger, C.B. Moore, The SBF survey of galaxy distances, IV: SBF magnitudes, colors, and distances. Astrophys. J. 546, 681-693 (2001). astro-ph/0011223. https://doi.org/10.1086/318301

R. Tripp, D. Branch, Determination of the Hubble constant using a two-parameter luminosity correction for type IA supernovae. Astrophys. J. 525, 209-214 (1999). astro-ph/9904347. https://doi.org/ $10.1086 / 307883$

R.B. Tully, J.R. Fisher, A new method of determining distances to galaxies. Astron. Astrophys. 54, 661-673 (1977)

A.V. Tutukov, L.R. Yungelson, Evolutionary scenario for close binary systems of low and moderate masses. Nauchn. Inf. 49, 3 (1981)

V.P. Utrobin, N.N. Chugai, Progenitor mass of the type IIP supernova 2005cs. Astron. Astrophys. 491, 507513 (2008). 0809.3766. https://doi.org/10.1051/0004-6361:200810272

J.P. Uzan, N. Aghanim, Y. Mellier, Distance duality relation from X-ray and Sunyaev-Zel'dovich observations of clusters. Phys. Rev. D 70(8), 083533 (2004). astro-ph/0405620. https://doi.org/10.1103/ PhysRevD.70.083533 
F. Vagnetti, S. Turriziani, D. Trevese, M. Antonucci, Variability and the X-ray/UV ratio of active galactic nuclei. Astron. Astrophys. 519, A17 (2010). 1005.0144. https://doi.org/10.1051/0004-6361/201014320

F. van Leeuwen, M.W. Feast, P.A. Whitelock, C.D. Laney, Cepheid parallaxes and the Hubble constant. Mon. Not. R. Astron. Soc. 379, 723-737 (2007). 0705.1592. https://doi.org/10.1111/ j.1365-2966.2007.11972.x

J.J. Walmswell, J.J. Eldridge, Circumstellar dust as a solution to the red supergiant supernova progenitor problem. Mon. Not. R. Astron. Soc. 419, 2054-2062 (2012). 1109.4637. https://doi.org/ 10.1111/j.1365-2966.2011.19860.x

I. Wanders, B.M. Peterson, D. Alloin et al., Steps toward determination of the size and structure of the broadline region in active galactic nuclei, XI: intensive monitoring of the ultraviolet spectrum of NGC 7469. Astrophys. J. Suppl. 113, 69-88 (1997). https://doi.org/10.1086/313054

B. Wang, Z. Han, Progenitors of type Ia supernovae. New Astron. Rev. 56, 122-141 (2012). 1204.1155. https://doi.org/10.1016/j.newar.2012.04.001

B. Wang, X. Meng, X. Chen, Z. Han, The helium star donor channel for the progenitors of type Ia supernovae. Mon. Not. R. Astron. Soc. 395, 847-854 (2009). 0901.3496. https://doi.org/10.1111/ j.1365-2966.2009.14545.x

F.Y. Wang, Z.G. Dai, Weak gravitational lensing effects on cosmological parameters and dark energy from gamma-ray bursts. Astron. Astrophys. 536, A96 (2011). 1112.4040. https://doi.org/10.1051/ 0004-6361/201117517

F.Y. Wang, Z.G. Dai, E.W. Liang, Gamma-ray burst cosmology. New Astron. Rev. 67, 1-17 (2015). 1504.00735. https://doi.org/10.1016/j.newar.2015.03.001

J.M. Wang, P. Du, C. Hu, H. Netzer, J.M. Bai, K.X. Lu, S. Kaspi, J. Qiu, Y.R. Li, F. Wang, SEAMBH Collaboration, Supermassive black holes with high accretion rates in active galactic nuclei, II: the most luminous standard candles in the universe. Astrophys. J. 793, 108 (2014). 1408.2337. https://doi.org/10.1088/0004-637X/793/2/108

L. Wang, Dust around type Ia supernovae. Astrophys. J. Lett. 635, L33-L36 (2005). astro-ph/0511003. https://doi.org/10.1086/499053

X. Wang, W. Li, A.V. Filippenko, R.J. Foley, N. Smith, L. Wang, The detection of a light echo from the type Ia supernova 2006X in M100. Astrophys. J. 677, 1060-1068 (2008a). 0711.2570. https:// doi.org/10.1086/529070

X. Wang, W. Li, A.V. Filippenko, K. Krisciunas, N.B. Suntzeff, J. Li, T. Zhang, J. Deng, R.J. Foley, M. Ganeshalingam, T. Li, Optical and near-infrared observations of the highly reddened, rapidly expanding type Ia supernova SN 2006X in M100. Astrophys. J. 675, 626-643 (2008b). 0708.0140. https://doi.org/10.1086/526413

D. Watson, K.D. Denney, M. Vestergaard, T.M. Davis, A new cosmological distance measure using active galactic nuclei. Astrophys. J. Lett. 740, L49 (2011). 1109.4632. https://doi.org/10.1088/ 2041-8205/740/2/L49

T.A. Weaver, S.E. Woosley, Evolution and explosion of massive stars, in Ninth Texas Symposium on Relativistic Astrophysics, ed. by J. Ehlers, J.J. Perry, M. Walker. Annals of the New York Academy of Sciences, vol. 336 (1980), pp. 335-357. https://doi.org/10.1111/j.1749-6632.1980.tb15942.x

G. Weigelt, K.H. Hofmann, M. Kishimoto, S. Hönig, D. Schertl, A. Marconi, F. Millour, R. Petrov, D. FraixBurnet, F. Malbet, K. Tristram, M. Vannier, VLTI/AMBER observations of the Seyfert nucleus of NGC 3783. Astron. Astrophys. 541, L9 (2012). 1204.6122. https://doi.org/10.1051/0004-6361/201219213

J.C. Wheeler, D. Pooley, Self-shielding of soft X-rays in type Ia supernova progenitors. Astrophys. J. 762, 75 (2013). 1208.0858. https://doi.org/10.1088/0004-637X/762/2/75

J. Whelan, I. Iben Jr., Binaries and supernovae of type I. Astrophys. J. 186, 1007-1014 (1973). https://doi.org/ $10.1086 / 152565$

O.C. Wilson, Possible applications of supernovae to the study of the nebular red shifts. Astrophys. J. 90, 634 (1939). https://doi.org/10.1086/144134

W.M. Wood-Vasey, G. Miknaitis, C.W. Stubbs, S. Jha, A.G. Riess, P.M. Garnavich, R.P. Kirshner, C. Aguilera, A.C. Becker, J.W. Blackman, S. Blondin, P. Challis, A. Clocchiatti, A. Conley, R. Covarrubias, T.M. Davis, A.V. Filippenko, R.J. Foley, A. Garg, M. Hicken, K. Krisciunas, B. Leibundgut, W. Li, T. Matheson, A. Miceli, G. Narayan, G. Pignata, J.L. Prieto, A. Rest, M.E. Salvo, B.P. Schmidt, R.C. Smith, J. Sollerman, J. Spyromilio, J.L. Tonry, N.B. Suntzeff, A. Zenteno, Observational constraints on the nature of dark energy: first cosmological results from the ESSENCE supernova survey. Astrophys. J. 666, 694-715 (2007). astro-ph/0701041. https://doi.org/10.1086/518642

T.E. Woods, M. Gilfanov, He II recombination lines as a test of the nature of SN Ia progenitors in elliptical galaxies. Mon. Not. R. Astron. Soc. 432, 1640-1650 (2013). 1302.5911. https://doi.org/10.1093/ mnras/stt586

S.E. Woosley, D. Kasen, Sub-Chandrasekhar mass models for supernovae. Astrophys. J. 734, 38 (2011). 1010.5292. https://doi.org/10.1088/0004-637X/734/1/38 
S.E. Woosley, T.A. Weaver, Sub-Chandrasekhar mass models for type IA supernovae. Astrophys. J. 423, 371-379 (1994). https://doi.org/10.1086/173813

X. Yang, H.R. Yu, T.J. Zhang, Constraining smoothness parameter and the DD relation of Dyer-Roeder equation with supernovae. J. Cosmol. Astropart. Phys. 6, 007 (2013). 1305.6989. https://doi.org/ 10.1088/1475-7516/2013/06/007

Y. Yang, L. Wang, D. Baade, P.J. Brown, M. Cracraft, P.A. Höflich, J. Maund, F. Patat, W.B. Sparks, J. Spyromilio, H.F. Stevance, X. Wang, J.C. Wheeler, Interstellar-medium mapping in M82 through light echoes around supernova 2014J. Astrophys. J. 834, 60 (2017). 1610.02458. https://doi.org/ 10.3847/1538-4357/834/1/60

O. Yaron, D. Prialnik, M.M. Shara, A. Kovetz, An extended grid of nova models, II: the parameter space of nova outbursts. Astrophys. J. 623, 398-410 (2005). astro-ph/0503143. https://doi.org/10.1086/428435

Y. Yoshii, The MAGNUM project: AGN variability as a new technique for distance determination, in New Trends in Theoretical and Observational Cosmology, ed. by K. Sato, T. Shiromizu (2002), p. 235

Y. Yoshii, Y. Kobayashi, T. Minezaki, S. Koshida, B.A. Peterson, A new method for measuring extragalactic distances. Astrophys. J. Lett. 784, L11 (2014). 1403.1693. https://doi.org/10.1088/2041-8205/ 784/1/L11

M. Young, M. Elvis, G. Risaliti, The X-ray energy dependence of the relation between optical and Xray emission in quasars. Astrophys. J. 708, 1388-1397 (2010). 0911.0474. https://doi.org/10.1088/ 0004-637X/708/2/1388

G. Zamorani, J.P. Henry, T. Maccacaro, H. Tananbaum, A. Soltan, Y. Avni, J. Liebert, J. Stocke, P.A. Strittmatter, R.J. Weymann, M.G. Smith, J.J. Condon, X-ray studies of quasars with the Einstein Observatory, II. Astrophys. J. 245, 357-374 (1981). https://doi.org/10.1086/158815 